\title{
HERMITIAN VECTOR BUNDLES AND VALUE DISTRIBUTION FOR SCHUBERT CYCLES(1)
}

\author{
BY
}

MICHAEL J. COWEN

ABSTRACT. R. Bott and S. S. Chern used the theory of characteristic differential forms of a holomorphic hermitian vector bundle to study the distribution of zeroes of a holomorphic section. In this paper their methods are extended to study how of ten a holomorphic mapping into a Grassmann manifold hits Schubert cycles of fixed type.

I. Introduction. Let $f: X \rightarrow Y$ be a holomorphic mapping of complex manifolds $X$ and $Y$, and let $W$ be a subvariety of $Y$ such that $f^{-1}(W)$ is a set of isolated points. Value distribution theory describes the behavior of $f^{-1}(W)$ when $X$ is noncompact and has an exhaustion by compact sets. For example, the theory gives conditions under which $f^{-1}(W)$ is nonempty. The central result of value distribution theory in several complex variables has been a First Main Theorem, an integral formula relating the size of $f^{-1}(W)$ with the growth of $f$. Wu [14] considered the case where $Y$ is either compact Kaehler or $\mathbf{C}^{n}$, and $W$ is a point. Hirschfelder [8] and Stoll [12] consider the following situation: Let $\tau, \sigma$ be proper, surjective, holomorphic, regular maps

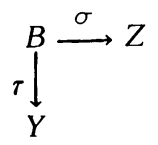

where $B, Z$ are complex manifolds, $Y$ is compact and $Z$ is compact Kaehler. Then $W=\tau\left(\sigma^{-1}(z)\right)$ for $z \in Z$. They allow $W$ to have arbitrary codimension and require $f^{-1}(W)$ to be an analytic set of codimension equal to codim $W$. Their result is proven by letting $\widetilde{X}=f^{*}(B, \tau)$, and considering the diagram

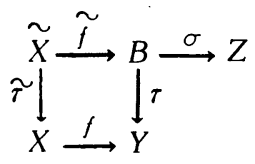

Received by the editors June 22, 1972.

AMS (MOS) subject classifications (1970). Primary 32H25; Secondary 53C55, 57D20.

Key words and phrases. First Main Theorem, Schubert cycle, Hermitian vector bundle, refined Chern class, Chern duality, ample vector bundle.

(1) The author's doctoral dissertation written under the supervision of Professor I. M. Singer and submitted to the Massachusetts Institute of Technology, June 1971. 
They then apply a generalization of Wu's results to the map $\tilde{f} \circ \sigma: \widetilde{X} \rightarrow Z$, and integrate over the fibres of $\widetilde{X}$. Bott and Chern [2] take a differential-geometric point of view: $Y$ is a holomorphic vector bundle $E$ over $X, f$ a holomorphic section $s$ of $E$, and $W$ the zero section of $E$.

We generalize the Bott-Chern situation as follows: Let $E$ be a holomorphic vector bundle of dimension $m$. Fix integers $0 \leq a_{1} \leq \cdots \leq a_{n} \leq m$ such that $\Sigma\left(m-a_{i}\right)=\operatorname{dim} X$ and put $Y=\bigoplus_{a_{n+n}} E, W=\left\{\left(e_{1}, \cdots, e_{a_{n}+n}\right) \in \bigoplus_{a_{n}+n} E \mid\right.$ rank $\left\{e_{1}, \cdots, e_{a_{i}+i}\right\} \leq a_{i}$ for $\left.1 \leq i \leq n\right\}$. A holomorphic section $\mathcal{S}$ of $\bigoplus_{a_{n}+n} E$ has a Scbubert zero of type $\left(a_{1}, \ldots, a_{n}\right)$ at $x \in X$ if $\mathcal{S}(x) \in W$; the Bott-Chern case is when $n=1, a_{1}=1$. We prove a First Main Theorem for Schubert zeroes, but only when $E$ has sufficiently many sections $V$, i.e. $V$ is a subspace of the space of holomorphic sections of $E, \operatorname{dim} V=m+n$, such that the sections in $V$ span the fibre $E_{x}$ for each $x$ in $X$. We first construct an analytic fibre bundle $\tilde{X}$ over $X$ and a holomorphic vector bundle $\widetilde{Q}$ over $\widetilde{X}$, fibre $\operatorname{dim} \widetilde{Q}=\operatorname{dim} \tilde{X}$. If $\mathcal{S} \in \bigoplus_{a_{n}+n} V$ is considered as a section of $\bigoplus_{a_{n}+n} E$, then $\mathcal{S}$ induces a holomorphic section $\widetilde{\mathcal{S}}$ of $\widetilde{Q}$, such that the Schubert zeroes of $\mathcal{S}$ are determined by the zeroes of $\widetilde{\mathcal{S}}$. We would like to apply the First Main Theorem of Bott and Chern to the section $\widetilde{\mathcal{S}}$, but $\tilde{X}$ is in general a singular analytic space. Hence we first show that the Bott and Chern theorem holds on analytic spaces. We then integrate the result over the fibres of $\tilde{X}$ to prove a First Main Theorem on $X$.

We could have approached the problem from the Stoll-Hirschfelder point of view, since $V$ induces a holomorphic map $e_{V}: V \rightarrow P_{n}(V)$, where $P_{n}(V)$ is the Grassmann manifold of $n$-planes of $V$. The map $e_{V}$ sends $x$ to the subspace of $V$ consisting of the sections in $V$ which vanish at $x$. Then we construct

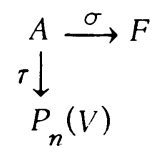

where $F$ is a flag manifold and $(A, \sigma),(A, \tau)$ are analytic fibre bundles over $F$

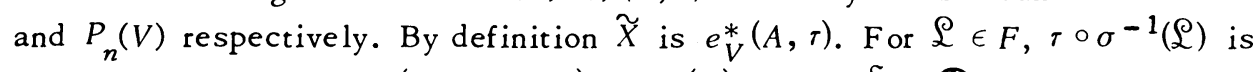
a Schubert cycle of type $\left(a_{1}, \cdots, a_{n}\right)$ in $P_{n}(V)$. Given $\mathcal{S} \in \bigoplus_{a_{n}+n} V$ we can define a flag $\mathcal{L}(\mathfrak{S}) \in F$ such that $\mathcal{S}$ has a Schubert zero at $x$ if and only if $e_{V}(x) \in$ $\tau\left(\sigma^{-1}(£(\mathcal{S}))\right)$. However, $A$ is not in general a manifold, so that we cannot immediately apply the First Main Theorem of Stoll and Hirschfelder. Further more, this situation is highly geometric, thus the Bott-Chern approach seems more natural in our case.

The plan of this paper is as follows: In §II we review the definition of characteristic forms of a holomorphic hermitian vector bundle. In particular we need to recall that a hermitian structure on the holomorphic bundle $E$ induces the Chern forms $c_{j}(E)$ of type $(j, j)$ for $j=1, \ldots, m=\operatorname{dim} E$. In $\S$ III we give a simplified proof of the Chern duality formula and use it to prove Bott and Chern's Theorem I: 
Let $E$ be a bolomorphic, bermitian vector bundle of fibre dimension $n$ over a complex manifold $X, \operatorname{dim} X=n$, and let $B^{*}(E)=\{e \in E \mid 0<\|e\|<1\}$ with $\pi$ : $B^{*}(E) \rightarrow X$ the projection. Then there exists a real form $\rho$ on $B^{*}(E)$ of type $(n-1, n-1)$ such that $\pi^{*} c_{n}(E)=d d^{c} \rho / 4 \pi$. Furtber if $E$ is nonnegative then $\rho$ may be chosen nonnegative.

(Note. $d^{c}=i(\bar{\partial}-\partial)$ ).

The Generalized Gauss-Bonnet Theorem and First Main Theorem of Bott and Chern are revised somewhat and shown to hold on analytic spaces in $\S I V$ and $\$ V$. The proof consists largely of integrating Theorem I.

We discuss Schubert zeroes and prove the First Main Theorem for Schubert zeroes in $\$ \mathrm{VI}$. Finally, in $\$$ VII we give sufficient conditions for almost all sections $\mathcal{S} \in \bigoplus_{a_{n}+n} V$ to have the same number of Schubert zeroes, or equivalently for a holomorphic map $f: X \rightarrow P_{n}(V)$ to hit almost all Schubert cycles the same number of times.

II. Hermitian vector bundles and characteristic classes. A connection on a $C^{\infty}$ vector bundle induces on the base manifold certain closed differential forms whose de Rham cohomology classes are independent of the original connection. When the connection is the canonical one defined by a hermitian structure on a holomorphic bundle, then the refined cohomology classes are independent of the hermitian structure and there is an explicit formula showing the behavior of these forms under deformation of the hermitian structure. The details and proofs are all found in Bott and Chern [2]; see Griffiths [6] for the principal bundle approach.

$E$ denotes an $n$-dimensional $C^{\infty}$-bundle over a $C^{\infty}$-manifold $X, T=T(X)$ is the tangent bundle, $A^{j}(X)$ is the space of complex valued differential $j$-forms, and $A(X)=\Sigma A^{j}(X)$. If $\Gamma(E)$ denotes the $C^{\infty}$-sections of $E$, then $A(X ; E)=A(X)$ $\otimes_{A^{0}(X)} \Gamma(E)$ is the space of differential forms on $X$ with values in $E$.

Definition. A connection on $E$ is a differential operator $D: \Gamma(E) \rightarrow$ $\Gamma\left(T^{*} \otimes E\right)$ such that $D(f s)=d f \cdot s+f \cdot D s$ whenever $f \in A^{0}(X), s \in \Gamma(E)$.

Relative to a fixed connection $D$ on $E$ we construct the Chern forms of $E$ as follows: Let $s=\left\{s_{i}\right\}, i=1, \ldots, n$, be a frame of $\left.E\right|_{U}, U$ open in $X$; i.e. the $s_{i}$ are smooth sections such that $\left\{s_{i}(x)\right\}$ form a bas is of $E_{x}$ for each $x \in U$. Then $D s_{i}=\Sigma \theta_{i j} s_{j}, \theta_{i j} \in A^{1}(U)$, and $\theta(s ; D)=\left\|\theta_{i j}\right\|$ is the matrix of connection 1 forms. The curvature matrix $K(s ; D)=\left\|K_{i j}\right\|$ is the matrix of 2 -forms defined by $K_{i j}=d \theta_{i j}-\Sigma_{k} \theta_{i k} \wedge \theta_{k j}$, i.e. $K(s, D)=d \theta(s, D)-\theta(s, D) \wedge \theta(s, D)$. We define a global form $c(E, D)=\Sigma c_{j}(E, D) \in A(X), c_{j}(E, D) \in A^{2 j}(X)$, by $\left.c(E, D)\right|_{U}=$ $\operatorname{det}(1+(i / 2 \pi) K(s ; D))$. This is well defined because it is independent of the choice of frame $s$ on $U$ and hence agrees on overlaps. If $s^{\prime}$ is another frame on $U, s_{i}^{\prime}=\Sigma A_{i j} s_{j}, A_{i j} \in A^{0}(U)$, i.e. $s^{\prime}=A s$, then $D s^{\prime}=\theta\left(s^{\prime}, D\right) s^{\prime}=(d A+A \theta(s, D))_{s}$, so $d A+A \theta(s, D)=\theta\left(s^{\prime}, D\right) A$ and hence $A K(s, D)=K\left(s^{\prime}, D\right) A$, so $\operatorname{det}(1+(i / 2 \pi) K(s, D))=$ 
$\operatorname{det}\left(1+(i / 2 \pi) K\left(s^{\prime}, D\right)\right)$. Note in particular the curvature matrix $K(s, D)$ represents an element $K[E, D]$, the curvature of $E$ relative to $D$, in $A^{2}(X, \operatorname{Hom}(E, E))$. The form $c(E, D)$ is the (total) Chern form, and $c_{j}(E, D)$ is the $j$ th Chern form, relative to $D$.

We define characteristic forms in a similar manner: Let $M_{n}$ denote the space of $n \times n$ complex matrices. A $k$-linear functional $\phi$ on $M_{n}$ is invariant if $\phi\left(x_{1}, \ldots, x_{k}\right)=\phi\left(y x_{1} y^{-1}, \ldots, y x_{k} y^{-1}\right)$ for all $y \in \mathrm{Gl}(n, \mathbf{C}), x_{i} \in M_{n}$. The space of all $k$-linear invariant forms will be denoted by $I_{k}\left(M_{n}\right)$. Given $\phi \in I_{k}\left(M_{n}\right), U$ open in $X$, we extend $\phi$ to a $k$-linear map $\phi_{U}: M_{n} \otimes A(U) \rightarrow A(U)$ by $\phi_{U}\left(x_{1} w_{1}, \ldots, x_{k} w_{k}\right)=\phi\left(x_{1}, \ldots, x_{k}\right) w_{1} \wedge \ldots \wedge w_{k}, x_{i} \in M_{n}, w_{i} \in A(U)$. Now consider $k$ elements $\xi_{i} \in A(X ; \operatorname{Hom}(E, E))$ and let $\phi \in I_{k}\left(M_{n}\right)$. Then there is a welldefined form $\phi\left(\xi_{1}, \ldots, \xi_{k}\right) \in A(X)$ which has the following local description: if $s$ is a frame over $U$ then $\left.\phi\left(\xi_{1}, \ldots, \xi_{k}\right)\right|_{U}=\phi_{U}\left(\xi_{1}(s), \ldots, \xi_{k}(s)\right)$ where $\xi_{i}(s)$ is the matrix of $\xi_{i}$ relative to $s$, and hence $\xi_{i}(s) \in M_{n} \otimes A(U)$. We set $\phi((\xi))=$ $\phi(\xi, \ldots, \xi)$; a characteristic form of the connection $D$ is $\phi((K[E, D]))$, for $\phi \epsilon$ $I_{k}\left(M_{n}\right)$. Polarizing the determinant we get det $\in I_{n}\left(M_{n}\right)$ by

$$
\operatorname{det}\left(X^{1}, \cdots, X^{n}\right)=\frac{1}{n !} \sum_{\tau, \sigma}(-1)^{\tau}(-1)^{\sigma} X_{\tau(1) \sigma(1)}^{1} \cdots X_{\tau(n) \sigma(n)}^{n}, \quad X^{j} \in M_{n},
$$

such that $\operatorname{det}((X))=\operatorname{det}(X)$. Thus the Chern form is a characteristic form. The desired independence on the connection of the characteristic (de Rham) classes follows from the following two propositions:

Proposition 2.1. Let $D$ be a connection for $E$ over $X, \phi$ an invariant form on $M_{n}$, then $\phi((K[E, D]))$ is closed.

Proposition 2.2. Let $D_{t}$ be a smooth 1-parameter family of connections on $E$, then the function $s \rightarrow d\left(D_{t} s\right) / d t, s \in \Gamma(E)$, is $A^{0}(X)$-linear and bence determines an element $\dot{D}_{t} \in A^{1}(X, \operatorname{Hom}(E, E))$. Further, if $\phi \in I_{k}\left(M_{n}\right)$ then

$$
\int_{a}^{b} \phi\left(\left(K\left[E, D_{t}\right]\right)\right)=d \int_{a}^{b} \phi^{1}\left(\left(K\left[E, D_{t}\right] ; \dot{D}_{t}\right)\right) d t
$$

where $\phi^{1}((\xi ; \eta))=\Sigma_{a} \phi\left(\xi, \ldots, \eta_{(a)}, \ldots, \xi\right)$.

Corollary 2.3. The cobomology class of $\phi((K[E, D]))$ is independent of the connection on $E$.

Proof. If $D_{1}$ and $D_{0}$ are two connections on $E$, then $D_{t}=t D_{1}+(1-t) D_{0}$ is a 1-parameter family of connections.

Remark. By putting $\theta(s, D)=0, s$ a frame of $U$, a connection can always be defined locally; patching together by a partition of unity shows the existence of connections on any bundle, so the characteristic classes are well defined topological invariants of the vector bundle. 
A real valued $C^{\infty}$ function $N: E \rightarrow \mathbf{R}$ defines a hermitian structure if the restriction of $N$ to any fibre defines a norm, i.e. $\langle u, v\rangle_{x}=1 / 2\{N(u+v)-N(u)-N(v)\}$ $+1 / 2\{N(u+i v)-N(u)-N(v)\}$ for $u, v \in E_{x}$, defines a hermitian inner product on $E_{x}$.

On a complex manifold $X$, let $A^{p, q}(X)$ be the forms of type $(p, q), d=\partial+\bar{\partial}$, $\partial: A^{p, q} \rightarrow A^{p+1, q}, \bar{\partial}: A^{p, q} \rightarrow A^{p, q+1} ; A^{r}(X)=\Sigma A^{p, q}(X), p+q=r$. This decomposition of $A(X)$ induces a corresponding decomposition of $A(X ; E)$ into $A^{p, q}(X ; E)=A^{p, q}(X) \otimes_{A}{ }^{0}(X) \Gamma(E)$ and hence any connection $D$ on $E$ splits canonically into the sum $D^{\prime}+D^{\prime \prime}$ where $D^{\prime}: \Gamma(E) \rightarrow A^{1,0}(X ; E), D^{\prime \prime}: \Gamma(E) \rightarrow$ $A^{0,1}(X ; E)$.

Proposition 2.4. Let $N$ be a bermitian norm on the bolomorphic bundle $E$. Then $N$ induces a canonical connection $D=D(N)$ on $E$ which is characterized by the two conditions:

(1) D preserves the norm $N$, i.e. $d\left\langle s, s^{\prime}\right\rangle=\left\langle D s, s^{\prime}\right\rangle+\left\langle s, D s^{\prime}\right\rangle, s, s^{\prime} \epsilon$ $\Gamma(E)$

(2) if $s$. is a bomomorphic section of $\left.E\right|_{U}$, then $D " s=0$ on $U$.

Note. $\left\langle s \otimes \theta, s^{\prime} \otimes \theta^{\prime}\right\rangle=\theta \wedge \bar{\theta}^{\prime} \cdot\left\langle s, s^{\prime}\right\rangle ; \theta, \theta^{\prime} \in A(U)$.

Locally $D(N)$ has the following form: let $s$ be a holomorphic frame on $U$, $N=N(s)=\left\|\left\langle s_{i}, s_{j}\right\rangle\right\|$. Then $\theta(s, D(N))=\partial N \cdot N^{-1}$.

Proof. See Bott-Chern [2].

Corollary 2.5. Let $E$ be a bolomorpbic bundle with bermitian norm $N$, and let $\theta, K$ denote the connection and curvature matrices of $D(N)$ relative to a bolomorphic frame over $U$. Then on $U$ one bas

$$
\begin{aligned}
& \theta \text { is of type }(1,0), \partial \theta=\theta \wedge \theta, \\
& K=\bar{\partial} \theta, \text { bence } K \text { is of type }(1,1) \text { and } \bar{\partial} K=0, \\
& \partial K=-[K, \theta] .
\end{aligned}
$$

Note. Since $K$ is of type $(1,1)$, the characteristic classes are of type $(p, p)$.

If $E$ is a line bundle, then a holomorphic frame is simply a nonvanishing section $s$, so $\theta=\partial \log N(s)$, and $c_{1}(E)=(i / 2 \pi) \bar{\partial} \partial \log N(s)$.

We define the refined cohomology groups $\hat{H}^{p, p}(X)=\operatorname{ker} d \cap A^{p, p}(X) / \delta \partial\left(A^{p-1, p-1}(X)\right)$ and wish to show that the refined characteristic classes are independent of the choice of norms.

Proposition 2.6. Let $N_{t}$ be a smooth family of norms on a bolomorphic bundle $E$. Then the function $\left\langle s, s^{\prime}\right\rangle=d\left\langle s, s^{\prime}\right\rangle_{N_{t}} / d t$ is hermitian linear over $A^{0}(X)$ and bence determines a section $L_{t} \in \Gamma(\operatorname{Hom}(E, E))$ by the formula $\left\langle L_{t}(s), s^{\prime}\right\rangle_{N_{t}}=$ $(d / d t)\left\langle s, s^{\prime}\right\rangle_{N_{t}}, s, s^{\prime} \in \Gamma(E)$. If $\phi$ is any invariant form in $I_{k}\left(M_{n}\right), n=\operatorname{dim} E$, then

$$
\int_{b}^{a} \phi\left(\left(K\left[E, N_{t}\right]\right)\right)=\bar{\partial} \partial \int_{a}^{b} \phi^{1}\left(\left(K\left[E, N_{t}\right] ; L_{t}\right)\right)
$$


where $\phi^{1}((\xi ; \eta))=\Sigma_{i} \phi\left(\xi, \ldots, \xi, \eta_{(i)}, \xi, \ldots, \xi\right)$.

Proof. See Bott-Chern [2].

Independence of the refined characteristic classes now follows by setting $N_{t}=$ $(1-t) N_{0}+N_{1}$ where $N_{0}$ and $N_{1}$ are norms. We let $\hat{c}(E)$ denote the refined Chern class of $E$, that is the refined class of $\hat{c}(E, N)$ for some norm $N$; a partition of unity argument shows that such an $N$ always exists, and hence $\hat{c}(E)$ is well-defined.

III. Chern duality. We wish to prove the Chern Duality formula: $\hat{c}(E)=$ $\hat{c}\left(E_{\mathrm{I}}\right) \hat{c}\left(E_{\mathrm{II}}\right)$ where $0 \rightarrow E_{\mathrm{I}} \rightarrow E \rightarrow E_{\mathrm{II}} \rightarrow 0$ is an exact sequence of holomorphic vector bundles. Let $N$ be a norm on $E$, then $N$ induces $N_{\mathrm{I}}$ on $E_{\mathrm{I}}$ by restriction and $N_{\mathrm{II}}$ on $E_{\mathrm{II}}$ by identifying $E_{\mathrm{I}}$ with $E_{\mathrm{I}}^{\perp}$, the orthogonal complement of $E_{\mathrm{I}}$, as $C^{\infty}$ bundles. Put $c(E)=c(E, N), c\left(E_{i}\right)=c\left(E_{i}, N_{i}\right)$ for $i=\mathrm{I}$, II then we have

Proposition 3.1. There exists a form $\xi$ in $A(X)$ sucb that

$$
c(E)-c\left(E_{\mathrm{I}}\right) c\left(E_{\mathrm{II}}\right)=\bar{\partial} \partial \xi
$$

The proof is a simplification of Bott and Chern's, and is based on a deformation of $N$ to $N_{\text {II }}$. First, we define the orthogonal projections $P_{i}: E \rightarrow E_{i}, i=$ $\mathrm{I}, \mathrm{II}$, and interpret them as elements of $\Gamma(\operatorname{Hom}(E, E))$. Now put $\langle u, v\rangle_{t}=$ $t\left\langle P_{\mathrm{I}} u, P_{\mathrm{I}} v\right\rangle+\left\langle P_{\mathrm{II}} u, P_{\mathrm{II}} v\right\rangle$ for $u, v \in E_{x}$ and $0<t \leq 1$. Then clearly $N_{t}$ is a hermitian norm and $N_{t} \rightarrow N_{\mathrm{II}}$ as $t \rightarrow 0$. We compute $K_{t}(E)$ at $x \in U$ relative to a holomorphic frame $s$ of $\left.E\right|_{U}$ such that $\left\{s_{1}, \ldots, s_{k}\right\}$ is a holomorphic frame of $E_{\mathrm{I}}$ and $s_{1}(x), \ldots, s_{n}(x)$ is an orthonormal basis of $E_{x}$. Write $N=N(s)=\left(\begin{array}{c}N_{I} A^{*} \\ A\end{array}\right)$ where $N_{\mathrm{I}}=\left\|\left\langle s_{i}, s_{j}\right\rangle\right\|_{i, j=1}^{k}, A=\left\|\left\langle s_{i+k}, s_{j}\right\rangle\right\|_{i=1, j=1}^{n-k} k^{k}, B=\left\|\left\langle s_{i+k}, s_{j+k}\right\rangle\right\|_{i, j=1}^{n-k}$ on $U$. Put $N_{\mathrm{II}}=N_{\mathrm{II}}\left(s^{\prime}\right)$ where $s^{\prime}$ is the holomorphic frame of $E_{\mathrm{II}}, s^{\prime}=\left\{P_{\mathrm{II}} s_{i+k}\right\}_{i=1}^{n-k}$. It is easy to check that $\left\|\left\langle P_{\mathrm{I}} s_{i+k}, P_{\mathrm{I}} s_{j+k}\right\rangle\right\|=A N_{\mathrm{I}}^{-1} A^{*}$. Hence

$$
\begin{aligned}
N_{t}=N_{t}(s) & =t\left\|\left\langle P_{\mathrm{I}} s_{i}, P_{\mathrm{I}} s_{j}\right\rangle\right\|+\left\|\left\langle P_{\mathrm{II}} s_{i}, P_{\mathrm{II}} s_{j}\right\rangle\right\| \\
& =t\left(\begin{array}{cc}
N_{\mathrm{I}} & A^{*} \\
A & A N_{\mathrm{I}}^{-1} A^{*}
\end{array}\right)+\left(\begin{array}{cc}
0 & 0 \\
& \\
0 & N_{\mathrm{II}}
\end{array}\right) .
\end{aligned}
$$

Note. At $x, A=A^{*}=0, N_{\mathrm{I}}=I_{k}, N_{\mathrm{II}}=I_{n-k}$ so $\partial\left(A N_{\mathrm{I}}^{-1} A^{*}\right)=\partial A N_{\mathrm{I}}^{-1} A^{*}-$ $A N_{\mathrm{I}}^{-1} \partial N_{\mathrm{I}} N_{\mathrm{I}}^{-1} A^{*}+A N_{\mathrm{I}}^{-1} \partial A^{*}=0, \bar{\partial} \partial\left(A N_{\mathrm{I}}^{-1} A^{*}\right)=-\partial A \bar{\partial} A^{*}+\bar{\partial} A \partial A^{*}$, and $N_{t}^{-1}=$ $\left(\begin{array}{cc}(1 / t) I_{k} & 0 \\ 0 & l_{n-k}\end{array}\right)$. Therefore at $x$ 


$$
\begin{gathered}
K\left[s, N_{t}\right]=\bar{\partial} \partial N_{t} N_{t}^{-1}+\partial N_{t} N_{t}^{-1} \bar{\partial} N_{t} N_{t}^{-1} \\
=\left(\begin{array}{cc}
t \bar{\partial} \partial N_{\mathrm{I}} & t \bar{\partial} \partial A^{*} \\
t \bar{\partial} \partial A & -t \partial A \bar{\partial} A^{*}+t \bar{\partial} A \partial A^{*}+\bar{\partial} \partial N_{\mathrm{II}}
\end{array}\right)\left(\begin{array}{cc}
(1 / t) I & 0 \\
0 & I
\end{array}\right) \\
\quad+\left(\begin{array}{cc}
t \partial N_{\mathrm{I}} & t \partial A^{*} \\
t \partial A & \partial N_{\mathrm{II}}
\end{array}\right)\left(\begin{array}{cc}
(1 / t) I & 0 \\
0 & I
\end{array}\right)\left(\begin{array}{cc}
t \bar{\partial} N_{\mathrm{I}} & t \bar{\partial} A^{*} \\
t \bar{\partial} A & \bar{\partial} N_{\mathrm{II}}
\end{array}\right)\left(\begin{array}{cc}
1 / t) I & 0 \\
0 & I
\end{array}\right) \\
=\left(\begin{array}{cc}
\bar{\partial} \partial N_{I}+\partial N_{\mathrm{I}} \bar{\partial} N_{\mathrm{I}}+t \partial A^{*} \bar{\partial} A & t \bar{\partial} \partial A+t \partial N_{\mathrm{I}} \bar{\partial} A^{*}+t \partial A^{*} \bar{\partial} N_{\mathrm{II}} \\
\bar{\partial} \partial A+\partial A \bar{\partial} N_{\mathrm{I}}+\partial N_{\mathrm{II}} \bar{\partial} A & t \bar{\partial} A \partial A^{*}+\bar{\partial} \partial N_{\mathrm{II}}+\partial N_{\mathrm{II}} \bar{\partial} N_{\mathrm{II}}
\end{array}\right) .
\end{gathered}
$$

But at $x, K\left[E_{\mathrm{I}}, N_{\mathrm{I}}\right]=\bar{\partial} \partial N_{\mathrm{I}}+\partial N_{\mathrm{I}} \bar{\partial} N_{\mathrm{I}}$ and $K\left[E_{\mathrm{II}}, N_{\mathrm{II}}\right]=\bar{\partial} N_{\mathrm{II}} \partial N_{\mathrm{II}}+\partial N_{\mathrm{II}} \bar{\partial} N_{\mathrm{II}}$, so putting $P_{i} K\left[E, N_{t}\right] P_{j}=K_{j i}\left[E, N_{t}\right], i, j=\mathrm{I}$, II, similarly for $K_{j i}[E, N]$, we have

$$
K\left[E, N_{t}\right]=\left(\begin{array}{cc}
K\left[E_{\mathrm{I}}, N_{\mathrm{I}}\right]+t \partial A * \bar{\partial} A & t K_{\mathrm{III}}[E, N] \\
K_{\mathrm{II} I}[E, N] & K\left[E_{\mathrm{II}}, N_{\mathrm{II}}\right]+t \partial A \partial A *
\end{array}\right) .
$$

The whole point is that

$$
K\left[E, N_{0}\right]=\left(\begin{array}{cc}
K\left[E_{\mathrm{I}}, N_{\mathrm{I}}\right] & 0 \\
K_{\mathrm{II} I}[E, N] & K\left[E_{\mathrm{II}}, N_{\mathrm{II}}\right]
\end{array}\right)
$$

so that

$$
\lim _{t \rightarrow 0} c\left(E, N_{t}\right)=\lim _{t \rightarrow 0} \operatorname{det}\left(I+(i / 2 \pi) K\left[E, N_{t}\right]\right)=c\left(E_{\mathrm{I}}\right) c\left(E_{\mathrm{II}}\right) .
$$

In order to apply Proposition 2.6 to this deformation, we compute $L_{t}$. Since $(d / d t)\langle u, v\rangle_{t}=\left\langle P_{\mathrm{I}} u, P_{\mathrm{I}} v\right\rangle=\left\langle(1 / t) P_{\mathrm{I}} u, v\right\rangle_{t}$, then $L_{t}=(1 / t) P_{\mathrm{I}}$. Thus

$$
\left.\right|_{t} ^{1} c\left(E, D_{t}\right)=\bar{\partial} \partial \int_{t}^{1} \operatorname{det}^{1}\left(\left(1+\kappa K\left[E, D_{t}\right] ; \kappa(1 / t) P_{\mathrm{I}}\right)\right) d t, \quad \kappa=i / 2 \pi
$$

for $0<t \leq 1$. If we could set $t=0$ we would be done; however, the integral will not in general converge. In fact, $K\left[E, N_{t}\right]=K\left[E, N_{0}\right]+t \Psi$, where $\Psi \epsilon$ $A^{2}(X ; \operatorname{Hom}(E, E))$ is independent of $t$. Therefore $\operatorname{det}^{1}\left(\left(1+\kappa K\left[E, N_{t}\right] ;(\kappa / t) P_{\mathrm{I}}\right)\right)=$ $\sum_{j=0}^{n-1} a_{j} t^{j-1}, a_{j} \in A(X)$; note these are the same $a_{j}$ 's as in Bott-Chern, i.e. the specific choice of deformation is immaterial. Now $a_{0}=\operatorname{det}^{1}\left(\left(1+\kappa K\left[E, N_{0}\right] ; \kappa P_{\mathrm{I}}\right)\right)$ so the integral converges if and only if $a_{0}=0$; but $a_{0}$ is a closed form, so it can be deleted from the integral. To see this, define $\operatorname{det}^{j}((\xi ; \eta))$ by $\operatorname{det}\left(\left(\xi+\lambda_{\eta}\right)\right)=$ $\Sigma \lambda^{j} \operatorname{det}^{j}((\xi ; \eta))$ for $\xi, \eta \in A^{1,1}(X, \operatorname{Hom}(E, E))$. But 


$$
\begin{gathered}
\operatorname{det}\left(\left(1+\kappa K\left[E, N_{0}\right]+\lambda \kappa P_{\mathrm{I}}\right)\right)=\operatorname{det}\left(\begin{array}{cc}
I+\lambda \kappa I+\kappa K\left[E_{\mathrm{I}}, N_{\mathrm{I}}\right] & 0 \\
K_{\mathrm{II} \mathrm{I}}[E, N] & I+\lambda K\left[E_{\mathrm{II}}, N_{\mathrm{II}}\right]
\end{array}\right) \\
=\operatorname{det}\left(I+\lambda \kappa I+\kappa K\left[E_{\mathrm{I}}, N_{\mathrm{I}}\right]\right) c\left(E_{\mathrm{II}}\right)=\left(\sum(1+\lambda \kappa)^{k-j} c_{j}\left(E_{\mathrm{I}}\right)\right) \cdot c\left(E_{\mathrm{II}}\right),
\end{gathered}
$$

so

$$
a_{0}=\operatorname{det}^{1}\left(\left(1+\kappa K\left[E, N_{0}\right] ; \kappa P_{\mathrm{I}}\right)\right)=\kappa\left(\sum_{j=0}^{k-1}(k-j) c_{j}\left(E_{\mathrm{I}}\right)\right) c\left(E_{\mathrm{II}}\right)
$$

which is closed. So now we can integrate from 0 to 1 and get $c(E)-c\left(E_{\mathrm{I}}\right) c\left(E_{\mathrm{II}}\right)=$ $\bar{\partial} \partial\left\{\Sigma_{\alpha=1}^{n-1} \alpha^{-1} \cdot a_{\alpha}\right\}$ and putting $\xi=\Sigma_{a=1}^{n-1} \alpha^{-1} a_{\alpha}$ we have $c(E)-c\left(E_{\mathrm{I}}\right) c\left(E_{\mathrm{II}}\right)=$ $\bar{\partial} \partial \xi$. Q.E.D.

In order to prove Theorem I (see Introduction) we must compute the highest degree term of $\xi$ in the case $\operatorname{dim} E_{\mathrm{I}}=k=1$, but this is just

$$
\begin{aligned}
\left(\kappa^{n} / t\right) \operatorname{det} & 1\left(\left(K\left[E, N_{0}\right]+t \Psi ; P_{\mathrm{I}}\right)\right) \\
= & \left(\kappa^{n} / t\right)\left\{\lambda^{1} \text { term of } \operatorname{det}\left(\begin{array}{cc}
K\left[E_{\mathrm{I}}, N_{\mathrm{I}}\right]+t \Psi_{\mathrm{II}}+\lambda & t \Psi_{\mathrm{I} \mathrm{II}} \\
K_{\mathrm{II} \mathrm{I}}[E, N] & K\left[E_{\mathrm{II}}, N_{\mathrm{II}}\right]+t \Psi_{\mathrm{II} \mathrm{II}}
\end{array}\right)\right\} \\
= & \left(\kappa^{n} / t\right) \operatorname{det}\left(K\left[E_{\mathrm{II}}, N_{\mathrm{II}}\right]+t \Psi_{\mathrm{II} \mathrm{II}}\right)=\kappa^{n} \sum \operatorname{det}^{j}\left(\left(K\left[E_{\mathrm{II}}, N_{\mathrm{II}}\right] ; \Psi_{\mathrm{II} \mathrm{II}}\right)\right) t^{j-1}
\end{aligned}
$$

where $\Psi_{\text {II II }}=K_{\text {II II }}[E, N]-K\left[E_{\mathrm{II}}, N_{\mathrm{II}}\right]=\bar{\partial} A \partial A^{*}$ in the notation of the proof of Proposition 3.1. So we have shown

Proposition 3.2. Let $0 \rightarrow E_{\mathrm{I}} \rightarrow E \rightarrow E_{\mathrm{II}} \rightarrow 0$ be an exact sequence of bolomorphic vector bundles, and let $c(E)$ and $c\left(E_{i}\right), i=\mathrm{I}$, II, denote the Chern forms induced by a norm $N$ on $E$. Then if $\operatorname{dim} E_{\mathrm{I}}=1, c_{n}(E)-c_{1}\left(E_{\mathrm{I}}\right)_{n-1}\left(E_{\mathrm{II}}\right)=$ $\kappa \bar{\partial}\left\{\Sigma_{j=1}^{n-1} \operatorname{det}^{j}\left(\left(\Omega\left[E_{\mathrm{II}}\right] ;-\Delta_{\mathrm{II}}[E]\right)\right)\right\}$ where $\Omega\left[E_{\mathrm{II}}\right]=\kappa K\left[E_{\mathrm{II}}, N_{\mathrm{II}}\right], \Omega_{\mathrm{II}}[E]=$ $\kappa K_{\mathrm{IIII}}[E ; N]$ and $-\Delta_{\mathrm{II}}=\Omega_{\mathrm{II}}[E]-\Omega\left[E_{\mathrm{II}}\right]$. Hence if $E_{\mathrm{I}}$ admits a nonvanisbing holomorphic section $s$, then

$$
c_{n}(E)=\kappa \bar{\partial} \partial\left\{\log N(s) c_{n-1}\left(E_{\mathrm{II}}\right)+\sum_{j=1}^{n-1} \operatorname{det}^{j}\left(\left(\Omega\left[E_{\mathrm{II}}\right] ; \Delta_{\mathrm{II}}[E]\right)\right)\right\} .
$$

Now, this proves Theorem I except for the positivity statement. In fact, let $\pi$ : $E \rightarrow X$ be the projection map; then $\pi^{*} E$, as a bundle over $E$, has a canonical section $s$ given by $s(e, x)=e$, for $e \in E_{x}$. If $B^{*}(E)$ denotes the set $\{e \in E \mid 0<N(e)$ 
$<1\}$, then $s$ is nonzero on $B^{*}(E)$ and letting $E_{\mathrm{I}}$ over $B^{*}(E)$ be the bundle spanned by $s$, we have $c_{n}\left(\pi^{*}(E)\right)=\kappa \bar{\partial} \partial \xi$ by the latter part of Proposition 3.2. But the Chern forms are functorial, so $\pi^{*} c_{n}(E)=\kappa \bar{\partial} \partial \xi$.

We must now discuss the "positivity" of $\xi$. Actually there are two notions of positivity we will use; both need only be defined pointwise. First, a form $a \epsilon$ $A^{p, p}(X)$ is strongly positive at $x$ if and only if there exist forms $\phi_{j} \in A^{p, 0}(X)$ such that $\alpha=i^{p^{2}} \Sigma_{j} \phi_{j} \wedge \bar{\phi}_{j}$ at $x$. A form $\alpha$ is said to be positive, written $\alpha \geq$ 0 , if and only if $i^{-p^{2}} \alpha\left(U_{1}, \cdots, U_{p}, \bar{U}_{1}, \ldots, \bar{U}_{p}\right) \geq 0$ for all $U_{1}, \ldots, U_{p} \in T_{x}^{1,0}(X)$, the holomorphic tangent space at $x$.

Remark 0. "Positive" is used to mean "nonnegative".

Remark 1. If $\alpha$ is strongly positive then $\alpha$ is positive.

Remark 2. If $\operatorname{dim} X=p$, i.e. if $\alpha$ is top dimensional, then in local coordinates we can write $a=i^{p^{2}} \mathfrak{Q} d z_{1} \wedge \ldots \wedge d z_{p} \wedge d \bar{z}_{1} \wedge \ldots \wedge d \bar{z}_{p}$ where $\mathbb{P} \in A^{0}(U)$; then $\alpha$ strongly positive $\Leftrightarrow a$ positive $\Leftrightarrow \mathbb{Q} \geq 0$.

Remark 3. $f: X \rightarrow Y$ holomorphic implies: if $\alpha \in A^{p, p}(Y)$ is positive (strongly positive), then $f^{*} \alpha$ is positive (strongly positive). It is easy to see that $\alpha \epsilon$ $A^{p, p}(X)$ is $\geq 0$ if and only if $f^{*} \alpha$ is $\geq 0$ for all holomorphic imbeddings $f: M \stackrel{!}{\rightarrow}$ $X, M$ a $p$-dimensional complex manifold.

We will show $\xi$ can be made strongly positive under suitable conditions on $E$, though in fact we only need $\xi \geq 0$. Later, when we fibre-integrate $\xi$, we will get a form which is only $\geq 0$, not strongly positive.

Definition. Let $\Omega$ be an $n \times n$ matrix of forms of type $(p, p)$. Then $\Omega$ is (strongly) positive at $x$, written $\Omega \geq 0$, if there exist $n \times m$ matrices $N_{r}$ of forms of type $(p, 0)$ at $x$ such that $\Omega=\left(i^{p^{2}} \Sigma_{r} N_{r} \wedge N_{r}^{*}\right)^{t}$.

Note. See Griffiths [6] for a discussion of positivity for $\Omega$ which generalizes positivity for forms (not strong positivity as we have done). This definition is the transpose of the one in Bott-Chern; but the bundles they say are positive are in fact not positive according to their definition.

If $A$ is an $n \times n$ nonsingular matrix then $\Omega \geq 0$ at $x$ if and only if $A \Omega A^{*} \geq$ 0 at $x$. So for $\xi \in A^{p, p}(X ; \operatorname{Hom}(E, E)), E$ a hermitian bundle, we say $\xi$ is positive $(\xi \geq 0)$ if the matrix of $\xi$, relative to an orthonormal base of $E_{x}$, is positive. This is well defined, since a unitary matrix has $A^{-1}=A^{*}$.

Definition. A holomorphic bundle $E$ with norm $N$ is positive if the real curvature form $\Omega[E, N] \equiv \kappa K[E ; N]$ is strongly positive on $X$.

Theorem 3.3. Let $E$ be a bermitian bundle of dimension $n, \xi^{r}$ positive elements of $A_{x}^{p, p}(X ; \operatorname{Hom}(E, E)), r=1, \cdots, n$. Then $\operatorname{det}\left(\xi^{1}, \ldots, \xi^{n}\right) \in A_{x}^{p n, p n}$ is $\geq 0$.

Proof. We can assume $\xi^{r}=\left({ }_{i}{ }^{2} N^{r} \wedge\left(N^{r}\right)^{*}\right)^{t}$, where $N^{r}=\left(N_{i j}^{r}\right)$ is an $n \times m_{r}$. matrix of $(p, 0)$ forms at $X$. Then 


$$
\begin{aligned}
& \operatorname{det}\left(\xi_{1}, \cdots, \xi_{n}\right)=\frac{1}{n !} \sum_{\sigma, \tau}(-1)^{\sigma}(-1)^{\tau} \xi_{\sigma(1) \tau(1)}^{1} \cdots \xi_{\sigma(n) \tau(n)}^{n} \\
& =\frac{1}{n !} i^{p^{2} n} \sum_{j_{i}, \cdots, j_{n}} \sum_{\sigma, t}(-1)^{\sigma}(-1)^{\tau} N_{\tau(1) j_{1}}^{1} \bar{N}_{\sigma(1) j_{1}}^{1} \cdots N_{\tau(n) j_{n}}^{n} \bar{N}_{\sigma(n) j_{n}}^{n} \\
& =i p^{2} n(-1)^{p^{2} n(n-1) / 2} \frac{1}{n !} \\
& \sum_{j_{1}}, \cdots, j_{n} \sum_{\sigma, \tau}(-1)^{\sigma}(-1)^{\tau} N_{\tau(1) j_{1}}^{1} \cdots N_{\tau(b) j_{n}}^{n} \bar{N}_{\sigma(1) j_{1}}^{1} \cdots \bar{N}_{\sigma(n) j_{n}}^{n} \\
& =\frac{1}{n !} i^{p^{2} n^{2}} \sum_{j_{1}, \cdots, j_{n}}\left(\sum_{\sigma}(-1)^{\sigma_{N}}{ }_{\sigma(1) j_{1}} \cdots N_{\sigma(n) j_{n}}^{n}\right) \\
& \wedge \overline{\left(\sum_{\sigma}(-1)^{\sigma} N_{\sigma(1) j_{1}}^{1} \cdots N_{\sigma(n) j_{n}}^{n}\right)} \\
& \geq 0
\end{aligned}
$$

in fact $\operatorname{det}\left(\xi^{1}, \ldots, \xi^{r}\right)$ is strongly positive.

Corollary 3.4. If $\xi, \eta \in A^{1,1}(X ; \operatorname{Hom}(E, E))$ are positive, then the forms $\operatorname{det}^{j}((\xi ; \eta))$ are positive. If $\xi \geq \xi^{\prime} \geq 0, \eta \geq \eta^{\prime} \geq 0$ then $\operatorname{det}^{j}((\xi ; \eta)) \geq \operatorname{det}^{j}\left(\left(\xi^{\prime} ; \eta^{\prime}\right)\right)$.

Proof. $\operatorname{det}^{j}((\xi ; \eta))$ can easily be seen to be $\operatorname{det}\left(\xi_{1}, \ldots, \xi_{n}\right)$ where $j$ of the $\xi_{i}$ 's are set equal to $\eta$, the rest to $\xi$. Also, $\operatorname{det}^{j}((\xi ; \eta))=\operatorname{det}^{j}\left(\left(\xi^{\prime}+\left(\xi-\xi^{\prime}\right) ; \eta^{\prime}+\left(\eta-\eta^{\prime}\right)\right)\right)=$ $\operatorname{det}^{j}\left(\left(\xi^{\prime} ; \eta^{\prime}\right)\right)+$ positive forms.

Note. In particular if $\Omega[E ; N] \geq 0$ for $E$ a hermitian bundle, then $c(E)$ is strongly positive.

Now consider again the sequence $0 \rightarrow E_{\mathrm{I}} \rightarrow E \rightarrow E_{\mathrm{II}} \rightarrow 0$ of holomorphic vector bundles. Define $\Omega_{i}[E], i=\mathrm{I}$, II to be the form $\kappa_{i} P_{i} K[E] P_{i}$ interpreted as a section of $A^{1,1}\left(X ; \operatorname{Hom}\left(E_{i}, E_{i}\right)\right)$. Then in the notation of the proof of Proposition 3.1 , at fixed $x \in U$, we have $\Omega\left[E_{\mathrm{I}}\right]-\Omega_{\mathrm{I}}[E]=-\kappa \partial A^{*} \bar{\partial} A, \Omega\left[E_{\mathrm{II}}\right]-\Omega_{\mathrm{II}}[E]=$ $-\kappa \bar{\partial} A \partial A^{*}$ so $\left(\Omega\left[E_{\mathrm{I}}\right]-\Omega_{\mathrm{I}}[E]\right)^{t}=-(1 / 2 \pi)\left(i \partial A^{*} \bar{\partial} A\right)^{t}$, i.e. $\left(\Omega\left[E_{\mathrm{I}}\right]-\Omega_{\mathrm{I}}[E]\right)^{t} \leq 0$ and $\Omega\left[E_{\mathrm{II}}\right]-\Omega_{\mathrm{II}}[E]=(1 / 2 \pi)\left(i \partial \bar{A} \bar{\partial} A^{t}\right)^{t} \geq 0$, i.e. subbundles are less positive, quotient bundles are more positive than the original bundle.

Now we have, in Proposition 3.2, $c_{n}(E)=\kappa \bar{\partial} \partial\left\{\log N(s)_{n-1}\left(E_{\mathrm{II}}\right)+\xi\right\}$, where $\xi=\sum_{i=1}^{n-1}(1 / i) \operatorname{det}^{i}\left(\left(\Omega\left[E_{\mathrm{II}}\right] ; \Omega_{\mathrm{II}}[E]-\Omega\left[E_{\mathrm{II}}\right]\right)\right)$. Assume $E$ is a positive bundle, i.e. $\Omega[E] \geq 0$; then $\Omega_{\mathrm{II}}[E] \geq 0$, so $\Omega\left[E_{\mathrm{II}}\right]=\Omega_{\mathrm{II}}[E]+\left(\Omega\left[E_{\mathrm{II}}\right]-\Omega_{\mathrm{II}}[E]\right) \geq 0$ which implies that $c_{n-1}\left(E_{\mathrm{II}}\right) \geq 0$. $\xi$ can be written as

$$
\sum_{i=1}^{n-1} \frac{1}{i}(-1)^{i} \operatorname{det}^{i}\left(\left(\Omega\left[E_{\mathrm{II}}\right] ; \Omega\left[E_{\mathrm{II}}\right]-\Omega_{\mathrm{II}}[E]\right)\right)
$$


which has indeterminate sign. Let $\xi_{0}=\sum_{i=1}^{n-1}(1 / i) \operatorname{det}^{i}\left(\left(\Omega\left[E_{\mathrm{II}}\right] ; \Omega\left[E_{\mathrm{II}}\right]\right)\right)$, then $\xi_{0}=\Sigma(1 / i)\left(\begin{array}{l}n \\ i\end{array} c_{n-1}\left(E_{\mathrm{II}}\right)\right.$ so $\xi_{0}$ is closed. But:

$$
\operatorname{det}^{i}\left(\left(\Omega\left[E_{\mathrm{II}}\right] ; \Omega\left[E_{\mathrm{II}}\right]\right)\right) \geq \operatorname{det}^{i}\left(\left(\Omega\left[E_{\mathrm{II}}\right] ; \Omega\left[E_{\mathrm{II}}\right]-\Omega_{\mathrm{II}}[E]\right)\right),
$$

so $\xi_{0}-\xi \geq 0$ and $\bar{\partial} \partial \xi_{0}=0$. Therefore,

$$
c_{n}=\left(d d^{c} / 4 \pi\right)\left\{\log N^{-1}(s) c_{n-1}\left(E_{\mathrm{II}}\right)+\left(\xi_{0}-\xi\right)\right\}
$$

and the bracketed term is $\geq 0$ whenever $\log N^{-1}(s) \geq 0$, i.e. when $N(s) \leq 1$. This completes the proof of Theorem I.

IV. Generalized Gauss-Bonnet on analytic spaces. The formula in Theorem I is integrated to give the Generalized Gauss-Bonnet Theorem. The proof is due to Bott and Chern, but an approximation which they use is not obvious, and thus has been given further elaboration. The proof of the Gauss-Bonnet Theorem is then seen to go through for the case of an analytic space.

Let $E$ be a holomorphic $n$-dimensional bundle with hermitian norm $N$, over the compact complex $n$-dimensional manifold $X$ with boundary $\partial X$. We consider $X-\partial X$ as an open subset of a complex $n$-manifold $Y$, with $\overline{X-\partial X}=X \subset Y$. Then $E$ and $N$ are actually assumed defined on the extension $Y$ of $X$. Letting $E_{0}=E$ minus the zero section and $\pi: E \rightarrow X$ the projection, we have already seen that the identity section of $\pi^{*} E$ over $E$ gives rise to a nonzero section on $E_{0}$ and formula (3.5) defines a definite form $\rho$ on $E_{0}$ such that $d d^{c} \rho / 4 \pi=\pi^{*} c_{n}(E)$. Putting $\eta(E)=d^{c} \rho / 4 \pi$ we have $\pi^{*} c_{n}(E)=d \eta(E)$.

Let $s$ be a smooth section of $E$ with an isolated zero at $x \in X$. We define the order of the zero at $x$, zero $(s, x)$, as follows: Let $B_{\epsilon}$ be a disc of radius $\epsilon>$ 0 relative to complex coordinates centered at $x$, and let $\psi:\left.E\right|_{B \delta} \rightarrow E_{x} \times B_{\delta}$ be a trivialization; $\phi:\left.E\right|_{B \delta} \rightarrow E_{x}$ is $\psi$ projected on the first factor. For small enough $\epsilon, \epsilon<\delta, \phi \circ s$ maps $\partial B_{\epsilon}$ into $E_{x}-\{0\}$ and zero $(s, x)$ is by definition the degree of $\phi \circ s: \partial B_{\epsilon} \rightarrow E_{x}-\{0\}$. Note. $B_{\epsilon}$ and $E_{x}-\{0\}$ are complex domains and have a canonical orientation, which induces orientations on $\partial B_{\epsilon}$ and the unit sphere $S\left(E_{x}\right) ; E_{x}-0$ can be retracted to $S\left(E_{x}\right)$, so the degree is well defined.

Proposition 4.1 (Generalized Gauss-Bonnet). Let $s$ be a smooth section of $E$ such that $s$ is nonvanishing on $\partial X$ and the zeroes of $s$ are isolated.

Then $\Sigma \operatorname{zero}(s, x)=\int_{X} c_{n}(E)-\int_{\partial X} s^{*} \eta(E)$ where $x$ ranges over the set of zeroes $x_{1}, \cdots, x_{m}$ of $s$.

Proof. Let $j_{x}: E_{x}-\{0\} \rightarrow E_{0}$ be inclusion, $x \in X$. Consider the form $j^{*} \eta(E)$. It is closed since $d\left(j^{*} \eta\right)=j^{*} c_{n}(E)=c_{n}\left(j^{*} E\right)$ and $j^{*} E$ is a trivial bundle with flat metric on $E_{x}-\{0\}$, so $c_{n}\left(j^{*} E\right)=0$. The cohomology class of $j^{*} \eta(E)$ is then a multiple of the orientation class of $E_{x}-\{0\}$ defined above, say $j^{*} \eta(E)=\alpha_{x}$. (orientation class), $\alpha_{x} \in R$. 
Let $X_{\epsilon}$ be $X$ minus the interiors of discs $B_{\epsilon}^{i}$ of radius $\epsilon$ around the $x_{i}$. Let $\delta$ be chosen small enough so that $s$ is nonvanishing on $X_{\epsilon}, 0<\epsilon \leq \delta$, hence $s$ defines a section of $E_{0}$ over $X_{\epsilon}$. Then $s^{*} \pi^{*} c_{n}(E)=c_{n}(E)$, so $c_{n}(E)=d s^{*} \eta(E)$ on $X_{\epsilon}$. Applying Stokes' Theorem,

$$
\int_{X_{\epsilon}} c_{n}(E)=\int_{\partial X_{\epsilon}} s^{*} \eta(E)=\int_{\partial X} s^{*} \eta(E)-\sum \int_{\partial E^{i}} s^{*} \eta(E) .
$$

Taking limits, we have

$$
\int_{X} c_{n}(E)-\int_{\partial X} s^{*} \eta=-\sum \lim _{\epsilon \rightarrow 0} \int_{\partial B_{\epsilon}^{i}} s^{*} \eta(E) .
$$

Assume further that $\delta$ is chosen small enough so that $\left.E\right|_{B_{\delta}}$ is trivial, and let $\phi_{i}$ : $\left.E\right|_{B_{\delta} i \rightarrow E_{x_{i}}}$ be trivializations as in the definition of zero $\left(s, x_{i}^{\delta}\right)$.

Claim. $\lim _{\epsilon \rightarrow 0} \int_{\partial B} i s^{*} \eta(E)=\lim _{\epsilon \rightarrow 0} \int_{\partial B_{\epsilon}^{i}} s^{*} \phi_{i}{ }^{*} j^{*} \eta(E)$. (This is not entirely clear, as asserted in Bott and Chern.) Let $N_{0}$ be a new hermitian norm, equalling $N$ on a neighborhood of $\partial B_{\delta}^{i}$, but with $N_{0}=\eta^{*} j^{*} N$ on $B_{\delta / 2}^{i}$. By the homotopy theorem, Proposition 2.6, there exists a $C^{\infty}$ form $\xi$ on $B_{\delta}^{i}$ such that $c_{n}(E, N)$ $c_{n}\left(E, N_{0}\right)=d \xi$ on $B_{\delta}^{i}$; by the construction of $\xi$ it is clear that $\xi \equiv 0$ on a neighborhood of $\partial B_{\delta}^{i}$. By Stokes' Theorem, $\int_{B_{\delta}} c_{n}(E, N)-c_{n}\left(E, N_{0}\right)=\int_{\partial B} i \xi=0$. Let $\eta_{0}=\eta\left(E, N_{0}\right)$ on $\left.E_{0}\right|_{B}{ }_{\delta}$. Then by the functoriality of $\eta, \eta_{0}=\phi^{*} j^{*} \eta(E, N)$ on $\left.E_{0}\right|_{B / 2} ^{i}$ Again by Stokes' Theorem,

$$
\begin{aligned}
0 & =\int_{B_{\delta}^{i}} c_{n}(E, N)-c_{n}\left(E, N_{0}\right) \\
& =\lim _{\epsilon \rightarrow 0} \int_{B_{\delta}^{i}-B_{\epsilon}^{i}} c_{n}(E, N)-c_{n}\left(E, N_{0}\right)=\lim _{\epsilon \rightarrow 0} \int_{B_{\delta}^{i}-B_{\epsilon}^{i}} s^{*} d \eta(E, N)-s^{*} d \eta\left(E, N_{0}\right) \\
& =\int_{\partial B_{\delta}^{i}}\left(s^{*} \eta(E, N)-s^{*} \eta\left(E, N_{0}\right)\right)-\lim _{\epsilon \rightarrow 0} \int_{\partial B_{\epsilon}^{i}}\left(s^{*} \eta(E, N)-s^{*} \eta\left(E, N_{0}\right)\right) \\
& =\int_{\partial B_{\delta}^{i}} i\left(s^{*} \eta(E, N)-s^{*} \eta(E, N)\right)-\lim _{\epsilon \rightarrow 0} \int_{\partial B_{\epsilon}^{i}}\left(s^{*} \eta(E, N)-s^{*} \phi^{*} j^{*} \eta(E, N)\right) \\
& =\lim _{\epsilon \rightarrow 0} \int_{\partial B_{\epsilon}^{i}}\left(s^{*} \phi^{*} j^{*} \eta-s^{*} \eta\right) .
\end{aligned}
$$

So $\lim _{\epsilon \rightarrow 0} \int_{\partial B_{\epsilon}^{i}} s^{*} \eta=\lim _{\epsilon \rightarrow 0} \int_{\partial B_{\epsilon}^{i}} s^{*} \phi^{*} j^{*} \eta$.

Therefore

$$
\begin{aligned}
\int_{X} c_{n}(E)-\int_{\partial X} s^{*} \eta & =-\sum \lim \int_{\partial B_{\epsilon}^{i}} s^{*} \eta=-\sum \lim _{\epsilon \rightarrow 0} \int_{\partial B_{\epsilon}^{i}} s^{*} \phi_{i} * j_{i} * \eta \\
& =-\sum \lim _{\epsilon \rightarrow 0} \int_{\partial B_{\epsilon}^{i}} s^{*} \phi_{i} *\left(\alpha_{i} \cdot \text { orientation class of } E_{x_{i}}-\{0\}\right) \\
& =-\sum \alpha_{i} \cdot \operatorname{zero}\left(s, x_{i}\right) .
\end{aligned}
$$


This completes the proof of Gauss-Bonnet, except for showing that $\alpha_{x_{i}}=-1$.

Lemma 4.2. $j^{*} \eta=-$ the orientation class of $E_{x}-\{0\}$ for $x \in X$; i.e. $\alpha_{x}=-1$.

Proof. Fix $x \in X$. By the functoriality of $\eta(E), j^{*} \eta$ depends only on the hermitian inner product on $E_{x}$, hence it suffices to exhibit a manifold $M$ without boundary and a holomorphic vector bundle $F$ over $M$ with hermitian norm such that $E_{x}$ is isometric to $F_{m}$ for some fixed $m \in M$ and $j_{m}{ }^{*} \eta(F)=-$ (the orientation class of $\left.F_{m}-\{0\}\right)$. Let us further assume that $M$ has a section $s$ of $F$ such that $s$ is zero only at $m$, zero $(s, m)=1$, and that $\int_{M} c_{n}(F)=1$. Then $\int_{M} c_{n}(F)-$ $\int_{\partial M} s^{*} \eta(F)=-\alpha_{m}$ zero $(s, m)$ so $1=-\alpha_{m}=-\alpha_{x}$, and we are done. Since these examples are needed for the result on Schubert cycles, we give the ir construction, found in Bott and Chern.

Let $V$ be a complex inner product space of dimension $m$ and $P_{n}(V)$ the Grassmann manifold of $n$-planes of $V$. Over $P_{n}(V)$ we have the canonical exact sequence

$$
0 \rightarrow S_{n}(V) \rightarrow P_{n}(V) \times V \rightarrow Q_{n}(V) \rightarrow 0
$$

of holomorphic vector bundles, with $S_{n}(V)$ the subbundle of $P_{n}(V) \times V$ consisting of all pairs $(A, v), A \in P_{n}(V), v \in V$ such that $v$ is an element of the $n$-plane $A$. The quotient bundle is $Q_{n}(V)$. The bundle $P_{n}(V) \times V$ is given the hermitian structure of $V$ in each fibre, and $S_{n}(V)$ and $Q_{n}(V)$ have the induced structures. Note that since $P_{n}(V) \times V$ has zero curvature, the bundle $Q_{n}(V)$ is (strongly) positive. Each $v \in V$ determines a holomorphic section $s_{v}$ of $Q_{n}(V)$ defined by the projection into $Q_{n}(V)$ of the constant section $x \rightarrow(x, v), x \in P_{n}(V)$, of $P_{n}(V) \times V$.

Assume that $m=n+1$. Consider the $n$-dimensional manifold $P_{1}(V)$ and the $n$-dimensional hermitian bundle $Q_{1}(V)$ over $P_{1}(V)$. If $v \neq 0 \in V$ then $s_{v}$ vanishes only at $\langle v\rangle$, the subspace of $V$ spanned by $v$. It is easy to check that zero $\left(s_{v},\langle v\rangle\right)=1$. Furthermore, we can choose the original hermitian structure on $V$ so that $V /\langle v\rangle$ has any given hermitian structure, for fixed $v \in V$, i.e. $\left(Q_{1}(V)\right)_{\langle v\rangle}$ has any given hermitian structure. To complete the proof of Lemma 4.2 we need only show that $\int_{P_{1}(V)} c_{n}\left(Q_{1}(V)\right)=1$. By Chern duality in the sequence $0 \rightarrow S_{1}(V)$ $\rightarrow P_{1}(V) \times V \rightarrow Q_{1}(V) \rightarrow 0$, we have $c\left(Q_{1}(V)\right) \cdot c\left(S_{1}(V)\right)=1$, so $c_{n}\left(Q_{1}(V)\right)=$ $(-1)^{n} c_{1}\left(S_{1}(V)\right)$ as cohomology classes. Let $\left\{v_{0}, \cdots, v_{n}\right\}$ be an orthonormal base for $v$, let $W=$ the span of $v_{1}, \cdots, v_{n}$ and put $U=P_{1}(V)-P_{1}(W)$; note $P_{1}(W)$ has measure zero in $P_{1}(V)$. The open set $U$ has coordinates $\left(z_{1}, \cdots, z_{n}\right) \rightarrow\left\langle v_{0}+\right.$ $\left.\sum_{i=1}^{n} z_{i} v_{i}\right\rangle$, the line spanned by $v_{0}+\sum_{i=1}^{n} z_{i} v_{i}$, and $v_{0}+\sum_{i=1}^{n} z_{i} v_{i}$ defines a nonvanishing section of $S_{1}(V) \mid U$ so $c_{1}\left(S_{1}(V)\right)=(i / 2 \pi) \bar{\partial} \partial \log N\left(v_{0}+\Sigma_{z_{i}} v_{i}\right)=$ $(i / 2 \pi) \bar{\partial} \partial \log \left(1+\|z\|^{2}\right)$ on $U$. Therefore

$$
\begin{aligned}
\int_{P_{1}(V)}{ }^{c}{ }_{n}\left(Q_{1}(V)\right) & =\int_{P_{1}(V)}(-1)^{n}\left(c_{1}\left(S_{1}(V)\right)\right)^{n} \\
& =\int_{U}(-1)^{n}\left(\frac{i}{2 \pi}\right)^{n}\left(\bar{\partial} \partial \log \left(1+\|z\|^{2}\right)\right)^{n} .
\end{aligned}
$$


Since $\left(\bar{\partial} \partial \log \left(1+\|z\|^{2}\right)\right)^{n}$ depends only on $\|z\|$, i.e. is invariant under the unitary group, we compute it at a point $z$ such that $z_{1}=\|z\|, z_{2}=\cdots=z_{n}=0$. Then

So

$$
\begin{aligned}
\left(\bar{\partial} \partial \log \left(1+\|z\|^{2}\right)\right)^{n} & =\left[\frac{d \bar{z}_{1} d z_{1}+\cdots+d \bar{z}_{n} d z_{n}}{1+\|z\|^{2}}-\frac{\|z\|^{2}}{\left(1+\|z\|^{2}\right)^{2}} d \bar{z}_{1} d z_{1}\right]^{n} \\
& =\left[\frac{d \bar{z}_{1} d z_{1}}{\left(1+\|z\|^{2}\right)^{2}}+\frac{d \bar{z}_{2} d z_{2}+\cdots+d \bar{z}_{n} d z_{n}}{1+\|z\|^{2}}\right]^{n} \\
& =\frac{n !}{\left(1+\|z\|^{2}\right)^{n+1}} d \bar{z}_{1} d z_{1} \cdots d \bar{z}_{n} d z_{n} .
\end{aligned}
$$

$$
\int_{P_{1}(V)}{ }^{c}{ }_{n}\left(Q_{1}(V)\right)=\int_{U}\left(\frac{i}{2 \pi}\right)^{n} \frac{n !}{\left(1+\|z\|^{2}\right)^{n+1}} d z_{1} d \bar{z}_{1} \cdots d z_{n} d \bar{z}_{n}
$$

and using polar coordinates, this is just

$$
\begin{gathered}
\frac{n !}{\pi^{n}} \int_{0}^{\infty}\left(\frac{1}{1+r^{2}}\right)^{n+1} r^{2 n-1} d r \cdot(\text { the volume of the unit } 2 n-1 \text { sphere) } \\
=\frac{n !}{\pi^{n} \cdot 2 n} \cdot \frac{\pi^{n} \cdot 2 n}{n !}=1 .
\end{gathered}
$$

(See Bott-Chern for another derivation of $\int c_{n}\left(Q_{1}(V)\right)=1$.) Q.E.D.

To see that the Generalized Gauss-Bonnet Theorem also holds on analytic spaces with boundary, we must first define differential forms, hermitian vector bundles, etc. on an analytic space. We define $A^{p, q}(X)$ for $X$ a complex analytic space, according to Bloom-Herrera [1]; see also King [10].

If $X$ is an analytic space, we denote by $R(X)$ the regular set of $X$. For $X$ a subvariety of a complex manifold $M$, let $J_{X} A^{p, q}(M)=\left\{\tau \in A^{p, q}(M) \mid i^{*} \tau=0\right\}$ where $i: R(X) \hookrightarrow M$ is inclusion, and define $A^{p, q}(X)=A^{p, q}(M) / J_{X} A^{p, q}(M)$. We define $A^{r}(X)$ similarly, and $A^{r}(X)=\Sigma_{p+q=r} A^{p, q}(X)$. Let $\tau \in A^{r}(M)$, then the following are equivalent (Bloom-Herrera):

(1) $i^{*} \tau=0$, where $i: R(X) \hookrightarrow M$,

(2) $g^{*} \tau=0$ for all smooth maps $g$, and all smooth manifolds $N, g: N \rightarrow M$ such that $g(N) \subseteq X$.

It is not obvious that (1) implies (2), for example when $g$ is a map of $N$ into the singular set of $X$, but this equivalence shows that $A^{p, q}(X)$ and $A^{r}(X)$ are independent of the imbedding. By patching together, we can then define $A^{p, q}(X)$ and $A^{r}(X)$ for any analytic space, and using property (2) it is clear that if $\tau \epsilon$ $A^{r}(Y)$ and $f: X \rightarrow Y$ is $C^{\infty}$ then we can define $f^{*} \tau \in A^{r}(X)$. If $\tau \in A^{p, q}(Y)$ and

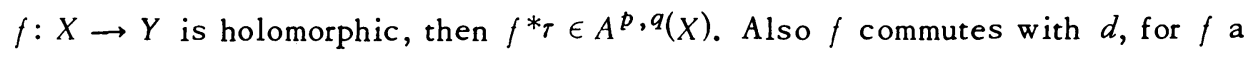
$C^{\infty}$ map, and with $\partial$ and $\bar{\partial}$ for $f$ holomorphic. 
A form $\tau \in A^{p, p}(X)$ is positive, $\tau \geq 0$, if $\tau$ restricted to $R(X)$ is positive. This is equivalent, just as (1) is equivalent to (2) above, to $g^{*} \tau \geq 0$ for all holomorphic maps $g$ and all complex manifolds $M, g: M \rightarrow X$. Hence if $X$ and $Y$ are analytic spaces, $f: X \rightarrow Y$ is holomorphic, and $\tau \in A^{p, p}(Y)$ is positive, then $f^{*} \tau \geq 0$. The definition of strong positivity for forms on analytic spaces is the same as that for forms on manifolds, i.e. $\tau \in A^{p, p}(X)$ is strongly positive if for each point $x \in X$, there exists a neighborhood $U$ of $x$ and forms $\theta_{j} \in A^{p, 0}(U)$ such that $\tau=$ ${ }_{i} p^{2} \Sigma \theta_{j} \wedge \bar{\theta}_{j}$ on $U$. Strong positivity is defined similarly for matrices of forms, see $\$$ III.

If $E$ is a holomorphic vector bundle over $X$, then $E$ is itself an analytic space, so the definition of a hermitian norm makes sense, and we can define the curvature matrix relative to a holomorphic frame, and hence define characteristic forms, just as we did in $\$ I I$. In order to show that Theorem I holds on analytic spaces, we need only show that we can define $\rho(E)$ a $C^{\infty}$ form on $E_{0}$ so that $\rho\left(\left.E\right|_{R(X)}\right)=$ $\left.\rho(E)\right|_{R\left(E_{0}\right)}$. But if $0 \rightarrow E_{\mathrm{I}} \rightarrow E \rightarrow E_{\mathrm{II}} \rightarrow 0$ is an exact sequence of holomorphic vector bundles on $X$ an analytic space, and $N$ is a hermitian norm on $E$, then $N_{\mathrm{I}}$ and $N_{\text {II }}$ are hermitian norms, and the orthogonal projections $P_{\mathrm{I}}$ and $P_{\text {II }}$ are smooth operators (the matrices of $N_{\text {II }}, P_{\mathrm{I}}, P_{\text {II }}$ relative to smooth frames are clearly smooth). Hence the deformation $N_{t}$, for $0<t \leq 1$, is a hermitian norm, so the form $\xi$ of Proposition 3.1, i.e. $\sum_{i=1}^{n-1} i^{-1} a_{i}$, where $\operatorname{det}^{1}\left(\left(1+\kappa K\left[E, N_{t}\right] ;(\kappa, t) P_{\mathrm{I}}\right)\right)=\sum_{i=0}^{n-1} a_{i} t^{i-1}$, is defined on $X$, and $c(E)-c\left(E_{\mathrm{I}}\right) c\left(E_{\mathrm{II}}\right)=\bar{\partial} \partial \xi$, since the restrictions to the regular set are equal by Proposition 3.1. The definition of a positive bundle is the same as before, so if $E \geq 0$ and we define $\rho(E)$ by

$$
\begin{aligned}
\rho(E)= & \log N^{-1}(s)_{n-1}\left(E_{\mathrm{II}}\right)+\sum_{j=1}^{n-1} \frac{1}{j} \operatorname{det}^{j}\left(\left(\Omega\left[E_{\mathrm{II}}\right] ; \Omega\left[E_{\mathrm{II}}\right]\right)\right) \\
& -\sum_{j=1}^{n-1} \frac{1}{j}(-1)^{j} \operatorname{det}^{j}\left(\left(\Omega\left[E_{\mathrm{II}}\right] ; \Omega\left[E_{\mathrm{II}}\right]-\Omega_{\mathrm{II}}[E]\right)\right)
\end{aligned}
$$

then $\rho(E) \geq 0$ on the space $B^{*}(E)=\{e \in E \mid 0<N(e)<1\}$ and $\pi^{*} c_{n}(E)=d d^{c} \rho / 4 \pi$, since this holds on the regular set.

In order to prove Gauss-Bonnet on analytic spaces, we must define spaces with boundary and show that Stokes' Theorem holds.

Definition. Let $Y$ be an $n$-dimensional space. Then $X \subset Y$ is an $n$-dimensional analytic space with boundary $\partial X$, if $X-\partial X$ is open in $Y, X$ is the closure in $Y$ of $X-\partial X$, and $X \cap R(Y)$ is an $n$-dimensional complex manifold with boundary $\partial X \cap R(Y)$. If $\mu \in A^{n, n}(Y), \nu \in A^{2 n-1}(Y)$, then we define $\int_{X} \mu, \int_{\partial X} \nu$ by $\int_{X \cap R(Y)} \mu$ and $\int_{\partial X \cap R(Y)} \nu$ respectively, if these integrals exist.

Remark. A smooth submanifold $M^{m}$ of $R(Y)$ has locally finite m-volume if $M \cap K$ has finite $m$-volume whenever $K$ is compact in $Y$. If $\mu \in A^{n, n}(Y)$ has compact support then $\int_{R(Y)} \mu$ always exists, since $R(Y)$ has locally finite $2 n$-volume; 
see Lelong [11] or Stolzenberg [13]. But if $X$ is an analytic space with boundary, $\partial X \cap R(Y)$ need not have locally finite $2 n-1$ volume.

Proposition 4.3 (Stokes' Theorem on Analytic Spaces). Let $Y$ be a paracompact complex analytic space of dimension $n$ and let $X \subseteq Y$ be an $n$-dimensional compact complex space with boundary $\partial X$ sucb that $\partial X \cap R(Y)$ bas locally finite $2 n-1$ volume. If $\tau \in A^{2 n-1}(Y)$ then $\int_{\partial X} \tau=\int_{X} d \tau$.

Proof. Since there exist $C^{\infty}$ partitions of unity on $Y$, the result need only be proved locally and follows from:

Proposition 4.4 (Stokes' Extended). Let $K$ be a compact subset of $\mathbf{R}^{m}, M a$ smooth $n$-manifold in $\mathbf{R}^{m}$ with locally finite $n$-volume, sucb that $\bar{M}-M$ (closure in $\mathbf{R}^{m}$ ) bas the following properties: $\bar{M}-M=\partial \cup \delta$ where

(i) $(M, \partial)$ is a (not necessarily compact) manifold with boundary.

(ii) $\partial \cap K$ bas finite $n-1$ volume.

(iii) $\delta \cap K$ is compact.

(iv) The image of $\delta$ under each coordinate projection $\left(x_{1}, \ldots, x_{m}\right) \rightarrow\left(x_{i_{1}}\right.$, $\left.\cdots, x_{i_{n}}\right)$ bas measure zero.

Then, for any $n-1$ form $\tau$ on $\mathbf{R}^{m}$ with support in $K$,

$$
\int_{\partial} \tau=\int_{M} d \tau
$$

Proof (Stolzenberg [13]). For each increasing multi-index $I,|I|=n-1$, let $\Pi_{I}: \mathbf{R}^{m} \rightarrow \mathbf{R}^{n-1}$ be the associated projection $\left(x_{1}, \cdots, x_{m}\right) \rightarrow\left(x_{i_{1}}, \cdots, x_{i_{n-1}}\right)$. Write $(\partial \cap K)_{I}=\partial \cap K-\Pi_{I}^{-1}\left(\Pi_{I}(\delta \cap K)\right)$ and $(M \cap K)_{I}=M \cap K-I_{I}^{-1}\left(\Pi_{I}(\delta \cap K)\right)$. For any smooth function $f$, with support in $K$, and integer $j \leq m$,

$$
\int_{\partial} f d x^{I}=\int_{(\partial \cap K)_{I}} f d x^{I} \text { and } \int_{M} f d x^{I} d x^{j}=\int_{M_{I}} f d x^{I} d x^{j} \text {. }
$$

For each $I$ choose a sequence of smooth functions $\Psi_{m}^{I}$ on $R^{n-1}$ such that $0 \leq \Psi_{m}^{I} \leq 1, \Psi_{m}^{I} \equiv 0$ on a neighborhood of $\Pi_{I}(\delta \cap K)$ and $\stackrel{m}{\Psi}_{m}^{I} \rightarrow 1$ uniformly on compact subsets of $\mathrm{R}^{n-1}-\Pi_{I}(\delta \cap K)$. Then set $\Phi_{m}^{I}=\Psi_{m}^{I} \circ \Pi_{I}$, write $\tau=\Sigma \tau_{I} d x^{I}$, and define $\tau_{m}=\Sigma \tau_{I} \Phi_{m}^{I} d x^{I}$. Since the form $\tau_{m}$ vanishes identically on (varying) neighborhoods of $\delta \cap K$, we can apply the usual Stokes' Theorem, so that $\int_{M} d \tau_{m}$ $=\int_{\partial} \tau_{m}$.

Now

$$
\tau_{m}=\sum \int_{\partial \cap K} \tau_{I} \Phi_{m}^{I} d x^{I}=\sum \int_{(\partial \cap K)_{I}} \tau_{I} \Phi_{m}^{I} d x^{I} \rightarrow \sum \int_{(\partial \cap K)_{I}} \tau_{I} d x^{I}=\int_{\partial} \tau
$$

Also, $d \tau_{m}=\Sigma \Phi_{m}^{I} d\left(\tau_{I} d x^{I}\right)$ since $d \Phi_{m}^{I} \wedge d x^{I}=\Pi_{I}^{*}\left(d \Psi_{m}^{I} \wedge d x_{1} \wedge \ldots \wedge d x_{n-1}\right) \equiv 0$. Thus

$$
\int_{M} d \tau_{m}=\sum \int_{(M \cap K)_{I}} \Phi_{m}^{I} d\left(\tau_{I} d x^{I}\right) \rightarrow \sum \int_{(M \cap K)_{I}} d\left(\tau_{I} d x^{I}\right)=\int_{M} d \tau
$$


Hence $\int_{M} d \tau=\int_{\partial} \tau$.

Remark. In the proof of Proposition 4.3, we let $\delta=X \cap \operatorname{Sing}(Y)$.

The proof of Gauss-Bonnet involved two parts: first applying Stokes' Theorem to $X$ minus little balls around the zeroes of $s$, then a local analysis at the zeroes. If the zeroes of $s$ are contained in the regular set of a complex analytic space, then the proof will still go through, since we have shown that Stokes' holds, so we have

Proposition 4.5 (Generalized Gauss-Bonnet on Analytic Spaces). Let $Y$ be a paracompact $n$-dimensional complex analytic space, and $X \subseteq Y$ a compact $n$-dimensional analytic space with boundary $\partial X$, such that $\partial X \cap R(Y)$ bas locally finite $2 n-1$ volume. Let $E$ be a bermitian, bolomorphic $n$-dimensional vector bundle over $Y$ and $s$ a smooth section of $E$ such that the zero set of $s$ intersected with $X$ is a set of isolated points contained in $(X-\partial X) \cap R(Y)$. Then $\Sigma$ zero $(s ; x)=$ $\int_{X} c_{n}(E)-\int_{\partial X} s^{*} \eta(E)$, where $x$ ranges over the set of zeroes $x_{1}, \cdots, x_{m}$ of $s$ in $X$.

V. The First Main Theorem. The Generalized Gauss-Bonnet Theorem of the previous section shows that if $X$ is a compact analytic space (without boundary) and $E$ is a holomorphic, hermitian vector bundle over $X$ with $n=\operatorname{dim} X=$ fibre $\operatorname{dim} E$, then a $C^{\infty}$ section of $E$ with isolated zeroes in $R(X)$ has exactly $\int_{X} c_{n}(E)$ number of zeroes. If $X$ is noncompact, then a $C^{\infty}$ section has no restriction on the number of zeroes. However, if $X$ has an exhaustion by compact analytic spaces with boundary, and the section $s$ is holomorphic then we can find the asymptotic distribution of zeroes by integrating the Gauss-Bonnet Theorem to get the First Main Theorem. The proof is due to Bott-Chern, except for Lemma 5.3, whose proof in Bott-Chern does not seem correct even in the dimension 1 case.

Definition. An exhaustion function $\tau$ on $X$ a complex analytic space is a $C^{\infty}$ real-valued function such that $\tau: X \rightarrow \mathbf{R}^{+}$is onto and $\tau$ is proper. Put $X_{r}=\{x \in$ $X \mid r(x) \leq r\}$.

Remark. If $\tau$ is an exhaustion on $X$ and $\sigma: Y \rightarrow X$ is proper and surjective, then $\tau \circ \sigma$ is an exhaustion on $Y$.

Fix $\tau$ an exhaustion on a complex space $X$. Sard's Theorem applied to $R(X)$ shows that $X_{r}$ is a compact analytic space with boundary $\partial X_{r}=\{x \in X \mid r(x)=r\}$ for almost all ${ }^{r}$ in $\mathbf{R}^{+}$. Since $X$ has locally finite $2 n$-volume, a theorem of Federer $\left[4\right.$, p. 188] shows that $\partial X_{r}$ has locally finite $2 n-1$ Hausdorff measure for almost all $r$. If $M^{m}$ is a submanifold of $\mathbf{R}^{p}$, then the $m$-dimensional Hausdorff measure of $M$ is the same as the $m$-volume of $M$, up to a constant (see Stolzenberg [13]). Hence there exists a set $\mathcal{C}$ in $\mathbf{R}^{+}$such that $\mathbf{R}^{+}-\mathcal{C}$ has measure zero, and for all $r \in \mathcal{C}, X_{r}$ is a compact analytic space whose boundary $\partial X_{r}$ has locally finite $2 n-1$ volume. 
Let $E$ be a holomorphic hermitian vector bundle of fibre dimension $n$ over $X$. The characteristic function $T(r)$ of $E$ is defined by $T(r)=\int_{0}^{r} \int_{X_{t}} c_{n}(E) d t$.

Let $s$ be a section of $E$ having only isolated zeroes contained in $R(X)$. The counting function $N(r, s)$ is defined by $N(r, s)=\int_{0}^{r}$ zero $\left(s, X_{t}\right) d t$ where zero $\left(s, X_{r}\right)=\Sigma$ zero $(s, x), x$ ranging over the zeroes of $s$ in the interior of $X_{r}$.

Theorem 5.1 (First Main Theorem). Let $X$ be a complex analytic space with exbaustion function $\tau$, and $E$ a positive bolomorphic bermitian vector bundle of fibre dimension $n=\operatorname{dim} X$. If $s$ is a bolomorphic section of $E$ with isolated zeroes in $R(X)$, and $\|s\| \leq 1$, then

$$
T(r)-N(r, s)=\frac{1}{4 \pi} \int_{\partial X_{r}} d^{c} \tau \wedge \lambda-\frac{1}{4 \pi} \int_{X_{r}} \lambda d d^{c} \tau
$$

where $\lambda=s^{*} \rho(E)$, for almost all $r$ (i.e. for $r \in \mathcal{C}$ ).

Proof (Bott-Chern). Let $\Gamma \subseteq R(X) \times \mathbf{R}$ be the graph of $\left.r\right|_{R(X)}$ and let $W$ be the region in $R(X) \times \mathbf{R}$ which is above $\Gamma$ and below $R(X) \times\{r\}, W=\{(x, t) \mid r(x) \leq t$ $\leq r, x \in R(X), t \in \mathbf{R}\}$, for fixed $r \in \mathcal{C}$. The natural projection $W \rightarrow X_{r}$ is denoted by $\sigma$. Then by Fubini it is clear that $T(r)=\int_{W} \sigma{ }^{*} c_{n}(E) d t$ with $W$ given the product orientation on $R(X) \times \mathbf{R}$ and $d t$ volume element on $\mathbf{R}$.

Suppose $s \neq 0$ on $X_{r}$, then $c_{n}(E)=(1 / 4 \pi)_{s} * d d^{c} \rho(E)$ on $X_{r}$, so $T(r)=$ $(1 / 4 \pi) \int_{W} d\left(\sigma^{*} s^{*} d^{c} \rho \wedge d t\right)$. The boundary of $W$ consists of $R\left(X_{r}\right) \times\{r\}$ and $\Gamma_{r}$, the graph of $\left.\tau\right|_{R\left(X_{r}\right)}$.

Note. $\Gamma$ has locally finite $2 n$-volume, since the injection of $R(X)$ into $\Gamma$, by $x \rightarrow(x, r(x))$, is locally lipschitzian and thus has locally finite Hausdorff measure; see Stolzenberg [13].

By choosing a partition of unity on $X \times \mathbf{R}^{+}$and applying Theorem 4.4, Stokes' Extended, we have $T(r)=(1 / 4 \pi) \int_{\partial W} \sigma^{*} s^{*} d^{c} \rho \wedge d t$. But $d t$ is zero on $X_{r} \times\{r\}$, so we have $T(r)=-(1 / 4 \pi) \int_{\Gamma_{r}} \sigma^{*} s^{*} d^{c} \rho \wedge d t$. Identifying $\Gamma_{r}$ with $R\left(X_{r}\right)$ we obtain

$$
T(r)=-\frac{1}{4 \pi} \int_{X_{r}} s^{*} d^{c} \rho \wedge d \tau=-\frac{1}{4 \pi} \int_{X_{r}} d^{c} s^{*} \rho \wedge d \tau .
$$

Keeping track of degrees it is easy to check that $d \tau \wedge d^{c} \lambda=d\left(\left(d^{c} \tau\right) \lambda\right)-\lambda d d^{c} \tau$ for any function $\tau$ and $(n-1, n-1)$ form $\lambda$. Using Stokes' Theorem once more, we obtain

$$
T(r)=\frac{1}{4 \pi} \int_{X_{r}} d\left(\left(d^{c} \tau\right) \wedge \lambda\right)-\lambda d d^{c} \tau=\frac{1}{4 \pi} \int_{\partial X_{r}} d^{c} \tau \wedge \lambda-\frac{1}{4 \pi} \int_{X_{r}} \lambda d d^{c} \tau,
$$

where $\lambda=s^{*} \rho(E)$, if $s$ has no zeroes in $X_{r}$.

Assume now that $s$ vanishes at isolated points $x_{1}, \cdots, x_{m}$ in $X_{r}$, where $x_{j}$ $\epsilon R(X), j=1, \cdots, m$, and we allow $x_{j}$ to be in $\partial X_{r}$. Let $X_{r}^{\epsilon}$ be $X_{r}$ minus discs 
$B_{i}(\epsilon)$ around the $x_{i}$, and let $W(\epsilon)$ be $W$ with the solid cylinders $C_{i}(\epsilon)$ above these discs removed. Then

$$
T(r)=\frac{1}{4 \pi} \lim _{\epsilon \rightarrow 0} \int_{W(\epsilon)} \sigma^{*} s^{*} d d^{c} \rho \wedge d t,
$$

and just as above we apply Stokes' Theorem to get

$$
\begin{aligned}
T(r)=\frac{1}{4 \pi} \lim _{\epsilon \rightarrow 0}\left\{\int_{\partial X_{r}^{\epsilon}} d^{c} \tau \wedge \lambda-\int_{X_{r}^{\epsilon}} \lambda \wedge d d^{c} \tau-\right. & \sum^{\prime} \int_{\partial C_{i}(\epsilon)} \sigma^{*} s^{*} d^{c} \rho \wedge d t \\
& \left.+\sum^{\prime \prime} \int_{\partial\left(C_{i}(\epsilon) \cap W\right)} \sigma^{*} s^{*} d^{c} \wedge d t\right\}
\end{aligned}
$$

where $\Sigma^{\prime}$ is the sum over the zeroes interior to $X_{r}$, and $\Sigma^{\prime \prime}$ the sum over the zeroes on $\partial X_{r}$. It is easy to show using the Generalized Gauss-Bonnet Theorem that

$$
\begin{aligned}
\lim _{\epsilon \rightarrow 0} & \int_{\partial C_{i}(\epsilon)} \sigma^{*} s^{*} d^{c} \rho \wedge d t \\
& =-\left(r-\tau\left(x_{i}\right)\right) \text { zero }\left(s, x_{i}\right) \text { if } x_{i} \text { interior to } X_{r}\left(\text { i.e. } \tau\left(x_{i}\right)<\mathrm{r}\right),
\end{aligned}
$$

and

$$
\lim _{\epsilon \rightarrow 0} \int_{\partial\left(C_{i}(\epsilon) \cap W\right)} \sigma^{*} s^{*} d^{c} \rho \wedge d t=0 \quad \text { if } x_{i} \in \partial X_{r} .
$$

But $N(r, s)=\Sigma\left(r-\tau\left(x_{i}\right)\right)$ zero $\left(s, x_{i}\right)$ so

(5.2) $T(r)-N(r, s)=\frac{1}{4 \pi} \lim _{\epsilon \rightarrow 0}\left[\int_{\partial X_{r}^{\epsilon}} d^{c} \tau \wedge \lambda-\int_{X_{r}^{\epsilon}} \lambda \wedge d d^{c} \tau\right], \quad \lambda=s^{*} \rho(E)$.

We apply the following lemma which will be proved below:

Lemma 5.3. (i) The form $\lambda d d^{c} \tau$ is absolutely integrable on $X_{r}$. (ii) The form $d^{c} \tau \wedge \lambda$ is absolutely integrable on $\partial X_{r}$. (iii) $\lim _{\epsilon \rightarrow 0} \int_{\partial B(\epsilon)}\left|d^{c} \tau \wedge \lambda\right|=0$.

Thus we may pass to the limit in (5.2) and have

$$
T(r)-N(r, s)=\frac{1}{4 \pi} \int_{\partial X_{r}} d^{c} \tau \wedge \lambda-\frac{1}{4 \pi} \int_{X_{r}} \lambda \wedge d d^{c} \tau . \quad \text { Q.E.D. }
$$

Corollary 5.4. Let $E$ be a positive hermitian bundle over an analytic space $X, n=\operatorname{dim} X=$ fibre $\operatorname{dim} E$, where $X$ bas exbaustion function $r$. Let $s$ be a bolomorphic section with isolated zeroes in the regular set of $X$, and $\|s\| \leq 1$. Then $N(r, s) \leq T(r)+(1 / 4 \pi) \int_{X_{r}} \lambda \wedge d d^{c} \tau$, for all $r \in \mathbf{R}^{+} ; \lambda=s^{*} \rho(E)$.

Proof (Bott-Chern). Since $E \geq 0$, by construction of $\rho(E)$ we have that $s^{*} \rho(E) \geq 0$ whenever $\|s\| \leq 1$. But the induced orientation on $\partial X_{r}$ for the Stokes' 
Theorem is characterized by: a real $(2 n-1)$ form $\xi$ on $X$ restricts to a positive form on $\partial X_{r}$, relative to the induced orientation, if and only if $d \tau \wedge \xi$ is positive on $X$ near $\partial X_{r}$. But if $\lambda$ is a positive $(n-1, n-1)$ form, then $d \tau \wedge d^{c} \tau \wedge \lambda$ is positive for any $C^{\infty}$ real valued function $\tau$, hence $\int_{\partial X_{r}} d^{c} \tau \wedge \lambda \geq 0$ where $\lambda=$ $s^{*} \rho(E)$. Thus for all $r \in \mathcal{C}$ we have $T(r)-N(r, s) \geq-(1 / 4 \pi) \int_{X_{r}} \lambda \wedge d d^{c} \tau$, but, by Lemma 5.3(i), $\int_{X_{r}} \lambda d d^{c} \tau$ is a continuous function of $r$, and certainly $T(r)$ and $N(r, s)$ are continuous in $r$, hence $N(r, s) \leq T(r)+(1 / 4 \pi) \int_{X_{r}} \lambda \wedge d d^{c} \tau$, for all $r$ in $\mathbf{R}^{+}$.

Definition. Let $E$ be a positive hermitian bundle over an analytic space $X$ with exhaustion $\tau$. If $c_{n}(E)>0$ at some point of $X$, then the deficiency of a holomorphic section $s$ with isolated zeroes in $R(X)$ is defined by

$$
\delta(s)=1-\varlimsup \lim \{N(r, s) / T(r)\} .
$$

Definition. An analytic space $X$ is pseudo-concave if it has an exhaustion function $\tau$ such that $d d^{c} \tau \leq 0$ for large values of $\tau$, i.e. there exists $r_{0} \cdot \ni \cdot d d^{c} \tau$ $\leq 0$ off $X_{r_{0}}$.

Corollary 5.5. Let $E$ be a positive hermitian bundle over a pseudo-concave analytic space $X$, with $c_{n}(E) \equiv 0$. If $s$ is a bolomorpbic section with isolated zeroes in $R(X)$ and $\|s\| \leq 1$, then $N(r, s) \leq T(r)+$ constant (constant depends on s) and bence $0 \leq \delta(s) \leq 1$.

Proof. Choose $r_{0} \cdot \exists \cdot d d^{c} \tau \leq 0$ off $X_{r_{0}}$, then $\lambda \wedge d d^{c} \tau \leq 0$ off $X_{r_{0}}$, for $\lambda=$ $s^{*} \rho(E)$, so $N(r, s) \leq T(r)+(1 / 4 \pi) \int_{X_{r_{0}}} \lambda \wedge d d^{c} \tau$.

We conclude this section with the proof of Lemma 5.3.

Proof of Lemma 5.3. Since $\lambda \geq 0$, we need only show (i) and (iii), because $d^{c} \tau \wedge \lambda$ is positive on $\partial X_{r}$ and (5.3) then shows $\lim _{\epsilon \rightarrow 0} \int_{\partial X_{r}^{\epsilon}} d^{c} \tau \wedge \lambda \rightarrow T(r)-$ $N(r, s)+(1 / 4 \pi) \int_{X_{r}} \lambda \wedge d d^{c} \tau$. We first investigate the local behavior of $\rho$, then we see how $\lambda=s^{*} \rho$ behaves. We are only concerned with the behavior of $\rho$ on $\left.E\right|_{R(X)}$, since $s$ has zeroes only in $R(X)$. Let $U$ be a coordinate patch of $R(X)$ with coordinates $z_{1}, \cdots, z_{n}$, such that $E$ has a holomorphic frame $s_{1}, \cdots, s_{n}$ on $U$. Then $\left.E\right|_{U}$ has coordinates $(z, w) \rightarrow \Sigma w_{i} s_{i}(z)$. Fix $\alpha \neq 0 \in \mathbf{C}$. Then $\alpha$ induces a map $\alpha: \dot{E}_{0} \rightarrow E_{0}$, multiplication by $\alpha$ on the fibre. We have already shown that

$$
\begin{gathered}
\rho=\log N^{-1}(s) c_{n-1}\left(E_{\mathrm{II}}\right)+\sum_{j>0} j^{-1} \operatorname{det}^{j}\left(\left(\Omega\left(E_{\mathrm{II}}\right) ; \Omega\left(E_{\mathrm{II}}\right)\right)\right) \\
-j^{-1}(-1)^{j} \operatorname{det}^{j}\left(\left(\Omega\left(E_{\mathrm{II}}\right) ; \Omega\left(E_{\mathrm{II}}\right)-\Omega_{\mathrm{II}}(E)\right)\right)
\end{gathered}
$$

where $s$ is the identity section of $\pi^{*} E$ over $E$. It is easy to see that ( $a^{*} E_{\mathrm{I}}$, $\left.a^{*} N_{\mathrm{I}}\right)=\left(E_{\mathrm{I}}, N_{\mathrm{I}}\right),\left(\alpha^{*} E_{\mathrm{II}}, a^{*} N_{\mathrm{II}}\right)=\left(E_{\mathrm{II}}, N_{\mathrm{II}}\right)$, and $\left(a^{*} E, a^{*} N\right)=(E, N)$, all as hermitian bundles. Therefore $\alpha^{*} c_{n-1}\left(E_{\mathrm{II}}\right)=c_{n-1}\left(E_{\mathrm{II}}\right), \alpha^{*} \Omega\left(E_{\mathrm{II}}\right)=\Omega\left(E_{\mathrm{II}}\right)$, and $\alpha * \Omega_{\mathrm{II}}(E)=\Omega_{\mathrm{II}}(E)$, but $N(s(z, w))=|\alpha|^{-2} N(s(z, \alpha w))$. Put $c_{\eta-1}\left(E_{\mathrm{II}}\right)=$ $\sum f_{I J K L}(z, w) d z^{I} d \bar{z}^{J} d w^{K} d \bar{w}^{L}$, and $\xi_{0}-\xi=\Sigma g_{I J K L}(z, w) d z^{I} d \bar{z}^{J} d w^{K} d \bar{w}^{L}$. Then 
it is clear that if we put $\alpha=1 /\|w\|$, then at $(z, w)$, with $w \neq 0$, we have:

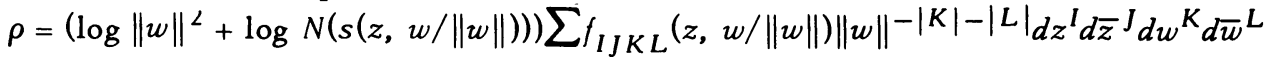

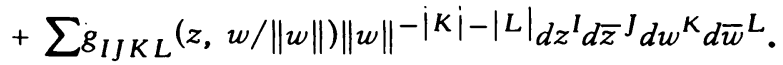

Let $s$ be a holomorphic section of $E$ with an isolated zero at $x \in R(X)$ and put $U=B_{\epsilon_{0}}$, with $\epsilon_{0}$ small enough that $E$ has a holomorphic frame $s_{1}, \ldots, s_{n}$. Then for $\epsilon<\epsilon_{0}$, the $f_{I J K L}(z, w /\|w\|), g_{I J K L}(z, w /\|w\|)$, and $N(s(z, w /\|w\|))$ are all bounded on $\left.E_{0}\right|_{B_{\epsilon}}$, and defining $f: B_{\epsilon_{0}} \rightarrow C^{n}$ a holomorphic function, by $s(z)=$ $\sum f_{i}(z)_{i}(z)$, we have that

$$
\lambda=\sum\left(\log \|f\|^{2} \phi_{I J K L}+\psi_{I J K L}\right)\|f\|^{-|K|-|L|} d z^{I} d \bar{z}^{J} d f{ }^{K} d \bar{f}^{L}
$$

where the $\phi_{I J K L}$ and $\Psi_{I J K L}$ are bounded functions on $B_{\epsilon}$.

The proof of (iii) involves Stokes' Theorem, thus we also want to describe $d \lambda$. Consider the form $d \rho$. Since $a^{*} d \log N^{-1}(s(z, w))=d \log |\alpha|^{2}+d \log N^{-1}(s(z, \alpha w))$ $=d \log N^{-1}(s(z, a w))$, then $\alpha^{*} d \log N^{-1}(s)=d \log N^{-1}(s)$ and hence $\alpha^{*} d \rho=d \rho$. Just as above we then have $d \lambda=\sum \theta_{I J K L}\|f\|^{-|K|-|L|} d z^{I} d \bar{z}^{J} d f{ }^{K} d \bar{f}^{L}$ where $|I|+$ $|J|+|K|+|L|=2 n-1$, and the $\theta_{1 J K L}$ are bounded functions on $B_{\epsilon}$.

Assume we have proven the following lemma:

Lemma 5.6. Let $f: B_{\epsilon} \rightarrow C^{n}$ be bolomorphic, with $f^{-1}(0)=0$. Then the $(n$, n) form $(\log \|f\|)\|f\|^{-|K|-|L|} d z^{I} d \bar{z}^{J} d f{ }^{K} d \bar{f}^{L}$ is absolutely integrable on $B_{\epsilon}$, where $|I|+|K|=n,|J|+|L|=n$, and $|K|+|L| \leq 2 n-1$.

From the description of $\lambda$ above, it is then clear that $\lambda \wedge \phi$ is absolutely integrable on $B_{\epsilon}$ for any smooth (1.1) form $\phi$, and hence (i) has been proven. In addition, we see that $d \lambda \wedge \Psi$ is absolutely integrable on $B_{\epsilon}$ for any smooth 1 -form $\Psi$.

Consider $\int_{B_{\epsilon}} \lambda \wedge \Psi$, for $\Psi$ a smooth 1 -form. Following an idea of Stoll [12], we let $g: \mathbf{R}^{+} \rightarrow \mathbf{R}^{+}$be a $C^{\infty}$ function such that $0 \leq g \leq 1, g \equiv 0$ on $[0,1 / 2]$, and $g \equiv 1$ on $[1, \infty)$. Define $\chi_{\delta}$ a real valued function on $B_{\epsilon}$ by $\chi_{\delta}(z)=g(\|f(z)\| / \delta)$. Then $\chi_{\delta}$ is $C^{\infty}, 0 \leq \delta \leq 1$, and satisfies the following conditions:

$$
\begin{gathered}
\chi_{\delta}(z)=1 \quad \text { if } \quad\|f(z)\| \geq \delta \\
\chi_{\delta}(z)=0 \quad \text { if } \quad\|f(z)\| \leq \delta / 2 \\
d_{\chi_{\delta}}=(1 / \delta) g^{\prime}(\|f\| / \delta) d\|f\|^{\circ}
\end{gathered}
$$

$$
=\frac{1}{(2 \delta)} g^{\prime}\left(\frac{\|f\|}{\delta}\right) \frac{\Sigma \overline{f_{i}} d f_{i}+\overline{f_{i}} d f_{i}}{\|f\|} \text {. }
$$

Note. $(1 / \delta) g^{\prime}(\|f\| / \delta)=0$ if $\|f\| \geq \delta$, hence

$$
\left|\frac{1}{(2 \delta)} g^{\prime}\left(\frac{\|f\|}{\delta}\right)\right| \leq \frac{1}{2\|f\|}\left|g^{\prime}\left(\frac{\|f\|}{\delta}\right)\right| \leq \frac{c}{\|f\|}
$$

where $c$ is a constant. 
Consider

$$
\begin{aligned}
\left|\lambda \wedge \chi \wedge d \chi_{\delta}\right| & \leq \frac{c}{\|f\|}\left|\lambda \wedge \Psi \wedge \frac{\Sigma f_{i} \overline{d f_{i}}+\overline{f_{i} d} f_{i}}{\|f\|}\right| \\
& \leq \frac{c}{\|f\|} \sum\left(\left|\lambda \wedge \Psi \wedge d f_{i}\right|+\left|\lambda \wedge \Psi \wedge \overline{d f_{i}}\right|\right)
\end{aligned}
$$

since $\left|f_{i}\right| /\|f\| \leq 1$. We can apply Lemma 5.6 to $\lambda \wedge \Psi \wedge d f_{i} /\|f\|$, and hence $\mid \lambda \wedge$ $\Psi \wedge d \chi_{\delta} \mid$ is dominated by an integrable form on $B_{\epsilon}$ for all $\delta$.

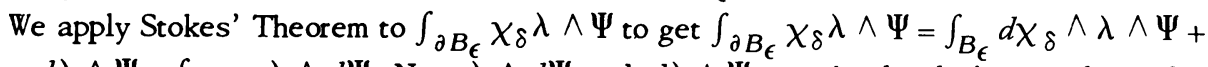
$\int_{B_{\epsilon}} \chi_{\delta} d \lambda \wedge \Psi+\int_{B_{\epsilon}} \chi_{\delta} \lambda \wedge d \Psi$. Now $\lambda \wedge d \Psi$ and $d \lambda \wedge \Psi$ are absolutely integrable, and $\chi_{\delta} \rightarrow 1$ as $\delta \rightarrow 0$. By the Dominated Convergence Theorem, we then have $\int_{B_{\epsilon}} d \chi_{\delta} \wedge \lambda \wedge \Psi$ $\rightarrow 0, \int_{B_{\epsilon}} \chi_{\delta} d \lambda \wedge \Psi \rightarrow \int_{B_{\epsilon}} d \lambda \wedge \Psi$, and $\int_{B_{\epsilon}} \chi_{\delta} \lambda \wedge d \Psi \rightarrow \int_{B_{\epsilon}} \lambda \wedge d \Psi$ as $\delta \rightarrow 0$. But $\left.\chi_{\delta}\right|_{B_{\epsilon}}=1$ for small enough $\delta$, hence $\int_{\partial B_{\epsilon}} \lambda \wedge \Psi=\int_{B_{\epsilon}} d \lambda \wedge \Psi+\int_{B_{\epsilon}} \lambda \wedge d \Psi$ which implies $\int_{\partial B_{\epsilon}} \lambda \wedge \Psi \rightarrow 0$ as $\epsilon \rightarrow 0$. This proves (iii).

Remark. If the Jacobian of $f$ is not zero at zero, then for purposes of convergence we can assume that $f$ is the identity map and only have to show that $(\log r) r^{-|K|-|L|} d z_{1} \cdots d z_{n} d \bar{z}_{1} \cdots d \bar{z}_{n}$ is absolutely integrable on $B_{\epsilon}$, where $|K|+|L| \leq$ $2 n-1$. But this is clear, since the volume of the sphere of radius $r$ is of order $r^{2 n-1}$ and

$$
\int_{0}^{\epsilon} \log r d r=\epsilon \log \epsilon-\epsilon .
$$

In order to prove the equidistribution theorems in $\S \mathrm{VII}$, we need only use Lemma 5.6 when the Jacobian of $f$ is nonzero at 0 . When the Jacobian of $f$ is zero, the result is quite difficult. Stoll, see [12], gives a very lengthy proof. The following argument, due to James King, is considerably shorter; but uses the deep result of Hironaka on resolving singularities.

Proof of Lemma 5.6. Step 1. Consider the subbundle $Y=S_{1}(V)$ contained in $P_{1}(V) \times V$ defined in $\S \mathrm{IV}, S_{1}(V)=\{(v, L) \mid v \in L, L$ a line in $V\}$, where $V=\mathbf{C}^{n}$. Let $\pi: Y \rightarrow V$ be the inclusion of $Y$ in $P_{1}(V) \times V$ followed by projection. Then the zero section of $Y$ is a projective space and equals $\pi^{-1}(0)$. The map $\left.\pi\right|_{Y-\pi-1(0)}$ is biholomorphic onto $V-\{0\}$; thus $\pi: Y \rightarrow V$ is the blow-up of zero in $V$. Let $z_{1}, \cdots, z_{n}$ be coordinates in $\mathrm{C}^{n}$; and let $U_{i} \subset P_{1}\left(\mathrm{C}^{n}\right)$ be the set $\{L \epsilon$ $\left.P_{1}\left(\mathrm{C}^{n}\right) \mid L=\left\langle\left(z_{1}, \ldots, z_{n}\right)\right\rangle, z_{i} \neq 0\right\}$. Put $W_{i}=\left.S_{1}(V)\right|_{U_{i}}$, then the $W_{i}$ are coordinate patches on $Y$, with coordinates $\left(t, w_{1}, \ldots, \hat{w}_{i}, \ldots, w_{n}\right) \rightarrow\left(t\left(w_{1}, \ldots, 1, \ldots, w_{n}\right)\right.$, $\left.\left\langle w_{1}, \ldots, 1, \ldots, w_{n}\right\rangle\right)$.

Let $\Gamma \tilde{f}$ denote the graph of $\tilde{f}$, where $\tilde{f}: B_{\epsilon}-\{0\} \rightarrow Y$ is the map induced by $\left.f\right|_{B_{\epsilon}-\{0\}}$. Let $\Gamma=\overline{\Gamma \tilde{f}}$, closure in $B_{\epsilon} \times Y$, then $\Gamma \subseteq \Gamma \tilde{f} \cup\left(\{0\} \times \pi^{-1}(0)\right)$. Now 
$\left(\Gamma \tilde{f} \cup\left(\{0\} \times \pi^{-1}(0)\right)\right) \cap w_{i}=\left\{\left(z, t, w_{1}, \ldots, \hat{w}_{i}, \ldots, w_{n}\right) \in B_{\epsilon} \times \mathbf{C}^{n} \mid t-f_{i}(z)=0\right.$, $\left.f_{i}(z) w_{j}-f_{j}(z)=0, j \neq i\right\}$, hence $\Gamma_{f} \cup\left(\{0\}_{\times} \pi^{-1}(0)\right)$ is an analytic space. Let $\left(w_{1}, \ldots, \hat{w}_{i}, \ldots, w_{n}\right)$ be an element of $\left(\{0\} \times \pi^{-1}(0)\right) \cap w_{i}$. Then it is easy to check since $\left.f\right|_{B_{\epsilon}}$ is an open map for small enough $\epsilon$, that there exists a sequence $z^{\alpha} \rightarrow 0$ such that $f_{i}\left(z^{\alpha}\right) \neq 0$ for each $\alpha$, and $f_{j}\left(z^{\alpha}\right) / f_{i}\left(z^{\alpha}\right) \rightarrow w_{j}$, for $j \neq i$. Thus $\Gamma \cap W_{i} \supset\left(\Gamma \tilde{f} \cup\left(\{0\} \times \pi^{-1}(0)\right)\right) \cap W_{i}$ so $\Gamma=\Gamma \tilde{f} \cup\left(\{0\} \times \pi^{-1}(0)\right)$. Note. $\Gamma$ is a $n$-dimensional irreducible analytic space.

Consider the map $\rho: \Gamma \rightarrow B_{\epsilon}$ defined by $\rho(z, \tilde{f}(z))=z, \rho\left(\{0\} \times \pi^{-1}(0)\right)=0$; then $\rho$ is a proper holomorphic map. By a partition of unity argument, in order to prove Lemma 5.6 it suffices to prove that

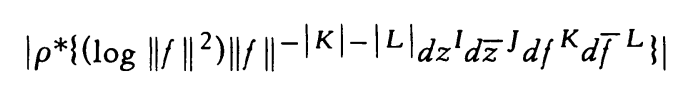

is locally integrable on $\Gamma$. We can assume that $I=(1, \ldots, n-|K|)$, so that we need only show

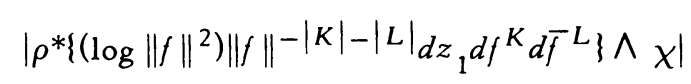

is locally integrable, where $\chi$ is a $C^{\infty}(n-|K|-1, n-|L|)$ form, and it clearly suffices to show the result on $\Gamma \cap W_{1}$.

On $\Gamma \cap w_{1}, \rho^{*}\|f\|^{2}=|t|^{2}\left(1+\left\|\left(w_{2}, \cdots, w_{n}\right)\right\|^{2}\right)=|t|^{2} g^{2}$, where $g \neq 0$ is locally bounded; $\rho^{*} d f_{1}=d t$; and $\rho^{*} d f_{i}=w_{i} d t+t d w_{i}$, for $2 \leq i \leq n$. Therefore,

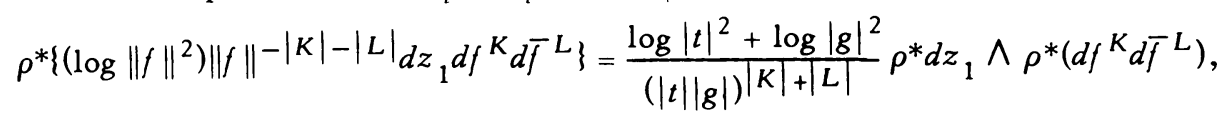

where

$$
\begin{aligned}
& \rho^{*} d f^{K} d \bar{f}^{L}=
\end{aligned}
$$

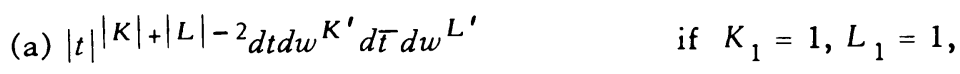

$$
\begin{aligned}
& \text { (b) }|t||K|+|L|-1 d w^{K} d \bar{t} d w L^{L^{\prime}}+\text { terms in (a) if } K_{1} \neq 1, L_{1}=1 \text {, } \\
& \text { (c) }|t||K|+|L|_{d w^{K}} d w^{L}+\text { terms in (a), (b) if } K_{1} \neq 1, L_{1} \neq 1 \text {. }
\end{aligned}
$$

Hence we need only show that

$$
\left|\frac{\log |t|}{|t|^{2}} d t d \bar{t} d z_{1} \chi_{1}\right|, \quad\left|\frac{\log |t|}{|t|} d \bar{t} \chi_{2}\right|, \text { and }|\log | t\left|\chi_{3}\right|
$$

are locally integrable, where $\chi_{1}, \chi_{2}, \chi_{3}$ are forms with locally bounded coefficients of type $(n-2, n-1),(n, n-1)$, and $(n, n)$ respectively.

Step 2. The subvariety $\{t=0\}$ of $\Gamma \cap w_{1}$ has codimension 1 , but is not in general a submanifold, i.e. $t$ is not a coordinate. Hence we resolve the singularities of $\Gamma \cap W_{1}$ to get an $n$-dimensional complex manifold $M$ and a proper holomorphic surjection $\phi: M \rightarrow \Gamma \cap W_{1}$ such that $\left.\phi\right|_{M-\phi-1\{0\}}$ is biholomorphic onto $\Gamma \cap W_{1}-\{t=0\}$, and $\phi^{-1}\{t=0\}$ is a divisor with normal crossings, i.e. there 
exist local coordinates $y_{1}, \cdots, y_{n}$ on $M$ such that $\phi^{-1}\{t=0\}=\left\{y_{1} \cdots y_{k}=0\right\}$ locally. (Actually we need only resolve around each point of $\{t=0\}$; see Hironaka [7].) Thus $\phi^{*} t=\alpha y_{1}^{a_{1}} \cdots y_{k}^{a_{k}}$ where $\alpha$ is never zero and is locally bounded; $a_{1}$, $\cdots, a_{k} \geq 1$.

It suffices to show that

$$
\left|\frac{\log \left|y_{r}\right|}{\left|y_{i}\right|\left|y_{j}\right|} d y_{i} d \bar{y}_{j} \phi^{*} d z_{1} \chi_{4}\right|, \quad\left|\frac{\log \left|y_{j}\right|}{\left|y_{i}\right|} \chi_{5}\right| \text { and }|\log | y_{i}\left|\chi_{6}\right|
$$

are locally integrable on $M$, where $\chi_{4}, \chi_{5}, \chi_{6}$ are forms with locally bounded coefficients of type $(n-2, n-1),(n, n)$, and $(n, n)$ respectively. But $\phi^{*} z_{1}$ vanishes on $\phi^{-1}\{t=0\}$, hence $\phi^{*} z_{1}=\beta y_{1} \cdots y_{k}$ where $\beta$ is locally bounded. Therefore $d y_{i} \phi^{*} d z_{1}=y_{1} \cdots y_{k} d y_{i} d \beta+\Sigma_{j \neq i} \beta y_{1} \cdots \hat{y}_{j} \cdots y_{k} d y_{i} d y_{j}$. Hence it actually suffices to show that $\left|\left(\left(\log \left|y_{j}\right|\right) /\left|y_{i}\right|\right) \chi_{5}\right|$ and $|\log | y_{i}\left|\chi_{6}\right|$ are locally integrable, which is clear since $\chi_{5}, \chi_{6}$ are of the form $g d y_{1} d \bar{y}_{1} \cdots d y_{n} d \bar{y}_{n}$, where $g$ is locally bounded. Q.E.D.

VI. Schubert zeroes. We define the Schubert zeroes of a set of sections of a holomorphic vector bundle $E$, and when $E$ has sufficiently many sections prove a First Main Theorem for Schubert zeroes. This can then be interpreted as a statement of how often a holomorphic map into a Grassmann manifold hits a fixed Schubert cycle.

Let $E$ be a holomorphic vector bundle over a complex space $X$. Fix integers $0 \leq a_{1} \leq \cdots \leq a_{n} \leq m=\operatorname{dim} E$ and let $\mathcal{S}=\left(s_{1}, \cdots, s_{n+a_{n}}\right)$ be a holomorphic section of $\bigoplus_{a_{n+n}} E$. A point $x$ in $X$ is a Schubert zero of $\stackrel{n}{\mathcal{S}}$, of type $\left(a_{1}, \cdots, a_{n}\right)$, if $\operatorname{rank}\left\{s_{1}(x), \ldots, s_{a_{i}+i}(x)\right\} \leq a_{i}$ for $i=1, \ldots, n$. This is of course the dual notion to the classical Schubert cycle. Let $V$ be a complex vector space of dimension $m+n, P_{n}(V)$ the Grassmann manifold of $n$-planes in $V$, and $\mathcal{\&}$ a flag $L_{a_{1+1}} \subset \ldots$ $\subset L_{a_{n}+n}$, where $L_{a_{i}+i}$ is a subspace of $V$ of dimension $a_{i}+i$. Then a Schubert cycle of type $\left(a_{1}, \cdots, a_{n}\right)$ of $P_{n}(V)$, which will be denoted by $\left(a_{1}, \ldots, a_{n}\right)(£)$, is defined by $\left(a_{1}, \cdots, a_{n}\right)(\stackrel{n}{\mathcal{E}})=\left\{L^{n} \in P_{n}(V) \mid \operatorname{dim}\left(L \cap L_{a_{i}+i}\right) \geq i, 1 \leq i \leq n\right\}$.

A Schubert cycle of type $\left(a_{1}, \ldots, a_{n}\right)$ is an analytic space of complex dimension $a_{1}+\cdots+a_{n}$, and if we fix a flag $L_{1} \subset \cdots \subset L_{m+n}=V, \operatorname{dim} L_{j}=j$, then the set of all Schubert cycles of $P_{n}(V)$, relative to this flag, give a cell decomposition of $P_{n}(V)$ into cells of even dimension; see Chern [3].

We give an example showing the connection between Schubert zeroes and Schubert cycles, which will later be used in proving the equidistribution theorem.

Definition. A holomorphic vector bundle $E$, of fibre dimension $m$ over a complex space $X$, has sufficiently many sections $V$ if $V$ is a finite dimensional subspare of the space of global holomorphic sections of $E$ and if we consider the evaluation map $e_{x}: V \rightarrow E_{x}$ which sends a section $s \in V$ into its value $s(x)$, then $e_{x}: V \rightarrow E_{x}$ is onto for each $x \in X$. 
Let $E$ have sufficiently many sections $V, \operatorname{dim} V=m+n$, and let $k(x)$ be the kernel of $e_{x}$. Then $k(x) \in P_{n}(V)$ and the map $x \rightarrow k(x)$ defines a holomorphic map $e_{V}: X \rightarrow P_{n}(V)$. Let $s_{1}, \cdots, s_{a_{n}+n}$ be independent sections in $V$, and define a flag $\mathcal{L}(\mathcal{S})$ in $V$ by putting $L_{a_{i}+i}(\mathcal{S})=\left\langle s_{1}, \ldots, s_{a_{i}+i}\right\rangle$, where $\langle, \ldots$,$\rangle denotes$ span. Then rank $\left\{s_{1}(x), \ldots, s_{a_{i}+i}(x)\right\} \leq a_{i}$ if and only if $k(x) \cap L_{a_{i}+i}$ has dimension $\geq i$; therefore $x \in X$ is a Schubert zero of $\mathcal{S}=\left(s_{1}, \ldots, s_{a_{n}+n}\right)$ of type $\left(a_{1}\right.$, $\left.\cdots, a_{n}\right)$ if and only if $e_{V}(x)$ is in the Schubert cycle $\left(a_{1}, \ldots, a_{n}\right)(\mathfrak{Q}(\mathcal{S}))$.

Alternately, let $f: X \rightarrow P_{n}(V)$ be a holomorphic map. We can assume that there is no $v \neq 0 \in V$ such that, for all $x \in X, v$ is in the $n$-plane $f(x)$. For if there are such $v$, put $W=\{v \mid v \in f(x)$ for all $x \in X\}, \operatorname{dim} W=p$, then $f$ induces $\tilde{f}: X \rightarrow P_{n-p}(V / W)$ and $\tilde{f}$ does satisfy the assumption. Let $\operatorname{dim} V=m+n$, and put $E=f^{*} Q_{n}(V)$, where $Q_{n}(V)$ is the $m$-dim quotient bundle defined in the proof of Lemma 4.2. Then each $v \in V$ induces a holomorphic section $s_{v}$ of $E$ and $V$ as a space of sections of $E$ has dimension $m+n$, since $v \neq 0$ implies $s_{v} \not \equiv 0$ by assumption. Let $\left(a_{1}, \cdots, a_{n}\right)(£)$ be a Schubert cycle in $P_{n}(V)$ and let $L_{a_{i}+i}$ be spanned by $v_{1}, \cdots, v_{a_{i}+i}$, for $1 \leq i \leq n$. Then $f(x) \in\left(a_{1}, \cdots, a_{n}\right)(£)$ if and only if $x$ is a Schubert zero of $\left(s_{1}, \cdots, s_{a_{n}+n}\right)$ where $s_{j}$ is the section induced by $v_{j}$. Thus, "hitting Schubert cycles in Grassmannians" and "having Schubert zeroes in bundles with sufficiently many sections" are equivalent notions.

Remark. There are two types of redundant conditions in the definition of Schubert cycles or zeroes:

(1) If $a_{i}=m$, then rank $\left\{s_{1}(x), \cdots, s_{a_{i}+i}(x)\right\} \leq a_{i}$ for all $x \in X$, and $\operatorname{dim}\left(L \cap L_{a_{i}+i}\right) \geq i$ for all $L \in P_{n}(V)$.

(2) If $a_{i}=a_{i+1}$, then rank $\left\{s_{1}(x), \ldots, s_{a_{i+1+i+1}}(x)\right\} \leq a_{i+1}$ implies $\operatorname{rank}\left\{s_{1}(x), \ldots, s_{a_{i}+i}(x)\right\} \leq a_{i}$, and $\operatorname{dim}\left(L \cap L_{a_{i+1}+i+1}\right) \geq i+1$ implies $\operatorname{dim}\left(L \cap L_{a_{i}+i}\right) \geq i$.

Hence, in order to maintain the equivalence of Schubert cycles and zeroes as constructed above, we may require the introduction of extraneous sections of $E$ (or subspaces in flags of $V$ ). For example, if $E$ has sufficiently many sections $V$, and $s_{1} \in V$, then the zeroes of $s_{1}$ are the same as the Schubert zeroes of $\left(s_{1}, \ldots\right.$, $\left.s_{m+n}\right)$ of type $(0, m, \cdots, m)$ for any sections $s_{2}, \cdots, s_{m+n}$. In the above equivalence we require $s_{1}, \cdots, s_{m+n}$ to be independent as sections, whereas for Schubert zeroes of type $(0, m, \cdots, m)$ it would be more natural to require that $s_{j}=0$ for $j>1$.

We now return to the case of an arbitrary holomorphic vector bundle $E$ over a complex space $X$, and define $E\left(a_{1}, \cdots, a_{n}\right)=\left\{\left(f_{1}, \cdots, f_{a_{n}+n}\right) \in \bigoplus_{a_{n}+n} E \mid\right.$ rank $\left\{f_{1}, \cdots, f_{a_{i}+i}\right\}>a_{i}$ for some $\left.i, 1 \leq i \leq n\right\}$. Then $E\left(a_{1}, \ldots, a_{n}\right)$ is an analytic $\mathrm{Gl}(m, \mathrm{C})$ fibre bundle over $X$ with fibre $\mathrm{C}^{m}\left(a_{1}, \ldots, a_{n}\right)$. 
Proposition 6.1.

$$
\tilde{H^{q}}\left(\mathbf{C}^{m}\left(a_{1}, \ldots, a_{n}\right)\right) \cong\left\{\begin{array}{l}
0 \text { if } q \leq 2 \sum_{1}^{n}\left(m-a_{i}\right)-2 \\
\mathbf{C} \text { if } q=2 \sum_{1}^{n}\left(m-a_{i}\right)-1
\end{array}\right.
$$

and the map $\Phi: \mathbf{C}^{\Sigma\left(m-a_{i}\right)}-\{0\} \rightarrow \mathbf{C}^{m}\left(a_{1}, \ldots, a_{n}\right)$ induces an isomorphism in cobomology in dimension $\leq 2 \Sigma\left(m-a_{i}\right)-1$, where $\Phi$ is defined as follows: let $\left\{e_{1}, \cdots, e_{m}\right\}$ be the standard basis for $\mathrm{C}^{m}$ and let $z=\left(z_{a_{1}+1}^{1}, \cdots, z_{m}^{1}, \cdots\right.$; $\left.z_{a_{n}+1}^{r}, \cdots, z_{m}^{r}\right)$ be coordinates for $\mathrm{C}^{\Sigma\left(m-a_{i}\right)}$, then $\Phi(z)=\left(e_{1}, \ldots, e_{a_{1}}\right.$, $\left.\Sigma_{a_{1+1}}^{m} z_{j}^{1} e_{j} ; \cdots ; e_{a_{n-1+1}}, \cdots, e_{a_{n}}, \Sigma_{a_{n+1}}^{m} z_{j}^{n} e_{j}\right)$.

Proof. Let $U=\mathrm{C}^{a_{n}+n}$ with basis $\left\{u_{1}, \cdots, u_{a_{n}+n}\right\}$, and $W=\mathrm{C}^{m}$ with the above basis $\left\{e_{1}, \cdots, e_{m}\right\}$. Put $V=U \oplus W$ and $K=P_{a_{n}+n}(V)$. Define $K_{1}=\{L \epsilon$ $K \mid \operatorname{dim}(L \cap W) \geq 1\}$ and $K_{2}=\left\{L \in K \mid \operatorname{dim}\left(L \cap\left\langle u_{1}, \cdots, u_{a_{i}+i}\right\rangle\right) \geq i\right.$ for all $1 \leq i$ $\leq n\}$. Then $K_{2}$ is a Schubert cycle of type $\left(a_{1}, \ldots, a_{n}, m, \ldots, m\right)$. We identify $\bigoplus_{a_{n}+n} W$ with $K-K_{1}$ by sending $\left(w_{1}, \ldots, w_{a_{n}+n}\right) \rightarrow\left\langle u_{1}+w_{1}, \ldots, u_{a_{n}+n}+\right.$ $\left.w_{a_{n+n}}\right\rangle$. Then under this identification, $W\left(a_{1}, \cdots, a_{n}\right)=K-K_{1} \cap K_{2}$. We would like to apply Mayer-Vietoris to the pair $\left\{K-K_{1}, K-K_{2}\right\}$, but in order to find the generators for the cohomology of $\left(K-K_{1}\right) \cap\left(K-K_{2}\right)=K-K_{1} \cap K_{2}$, we must first find generators for the cohomology of $K-K_{2}$ and $K-K_{1} \cap K_{2}$. From the Schubert cell decomposition of $K$, we have that $H^{q}(K) \rightarrow H^{q}\left(K_{2}\right)$ is surjective, and

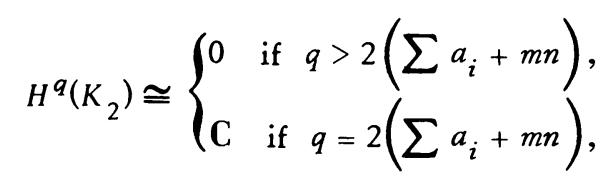

so by duality:

(i) $H_{p}\left(K-K_{2}\right) \cong H_{p}(K)$ if $p<2 m\left(a_{n}+n\right)-2\left(\Sigma a_{i}+m n\right)=2 \Sigma\left(m-a_{i}\right)$ and $0 \rightarrow H_{p}\left(K-K_{2}\right) \rightarrow H_{p}(\stackrel{p}{K}) \rightarrow \mathrm{C} \rightarrow 0$ is exact if $p<2 \Sigma\left(m-a_{i}\right)$.

Since $K_{2}$ is an irreducible analytic variety in $K$, and $K_{1}$ has codim 1 in $K$, we see that $K_{1} \cap K_{2}$ is a variety of dimension $\Sigma a_{i}+m a_{n}-1$, hence $H^{2 m\left(a_{n}+n\right)-p}\left(K_{1} \cap K_{2}\right) \cong 0$ if $p<2 \Sigma\left(m-a_{i}\right)+2$ so aga in by duality

(ii) $H_{p}\left(K-K_{1} \cap K_{2}\right) \cong H_{p}(K)$ if $p \leq 2 \sum^{i}\left(m-a_{i}\right)$.

Consider the flag $L_{1} \subset \cdots \subset L_{a_{n}+n+m}=V$ where $L_{j}$ is the $j$-dimension space spanned by the first $j$ of

$$
\begin{aligned}
& \left\{e_{1}+u_{1}, \ldots, e_{a_{1}}+u_{a_{1}} ; \ldots ; e_{a_{i}+1}+u_{a_{i}+i+1}, \ldots, e_{a_{i+1}}+u_{a_{i+1}+i}\right. \\
& \left.\quad \ldots ; e_{a_{n-1}+1}+u_{a_{n-1}+n}, \cdots, e_{a_{n}}+u_{a_{n}+n} ; e_{a_{n}+1}, \cdots, e_{m} ; u_{a_{n}+n}, \cdots, u_{1}\right\} .
\end{aligned}
$$


All Schubert cycles will be with respect to this flag. Then if $\left(b_{1}, \cdots, b_{a_{n}+n}\right)$ is a Schubert cycle with $\Sigma_{1}^{a_{n}+n} b_{j} \leq \Sigma_{1}^{n}\left(m-a_{i}\right)$ then

$\left(b_{1}, \ldots, b_{a_{n}+n}\right) \cap K_{2}= \begin{cases}\varnothing \quad \text { if }\left(b_{1}, \ldots, b_{a_{n}+n}\right) \neq\left(0, \ldots, 0, m-a_{n}, \ldots, m-a_{1}\right), \\ \{\alpha\} \quad \text { if }\left(b_{1}, \ldots, b_{a_{n}+n}\right)=\left(0, \ldots, 0, m-a_{n}, \ldots, m-a_{1}\right),\end{cases}$

with $\alpha=\left\langle L_{a_{n}}, u_{a_{1+1}}, \cdots, u_{a_{n}+n}\right\rangle \in P_{a_{n}+n}(V)=K$. Let $K_{3}$ be the union of all Schubert cells (relative to the above flag) of $\operatorname{dim} \leq \Sigma\left(m-a_{i}\right)$. Then $K_{3}-\{a\} \subseteq$ $K-K_{2}$, and since $a \notin K_{1}$, then $K_{3} \subseteq K-K_{1} \cap K_{2}$.

Remark. The obvious choice of flag would have been for $L_{j}$ to be spanned by the first $j$ of $\left\{e_{1}, \ldots, e_{m}, u_{a_{n}+n}, \cdots, u_{1}\right\}$ but then $K_{2} \cap\left(0, \ldots, 0, m-a_{n}\right.$, $\left.\cdots, m-a_{1}\right)$ would lie in $K_{1}$, and we would not have $K_{3} \subseteq K-K_{1} \cap K_{2}$; hence we shift the flag.

Since $H^{p}(K) \cong H^{p}\left(K_{3}\right)$ if $p \leq 2 \Sigma\left(m-a_{i}\right)$ we have by (ii):

(iii) $H^{p}\left(K-K_{1} \cap K_{2}\right) \cong H^{p}\left(K_{3}\right)$ if $p \leq 2 \Sigma\left(m-a_{i}\right)$.

But $K_{3}-\{\alpha\}$ is homotopic to $K_{3}$ minus an open Schubert cell of dimension $\Sigma\left(m-a_{i}\right)$, so that $H_{p}\left(K_{3}-\{\alpha\}\right) \cong H_{p}(K)$ if $p<2 \Sigma\left(m-a_{i}\right)$, and $0 \rightarrow$ $H_{p}\left(K_{3}-\{\alpha\}\right) \rightarrow H_{p}(K) \rightarrow \mathrm{C} \rightarrow 0$ is exact if $p=2 \Sigma\left(m-a_{i}\right)$. By (i) we have

(iv) $H^{p}\left(K-K_{2}\right)=H^{p}\left(K_{3}-\{a\}\right)$ for $p \leq 2 \Sigma\left(m-a_{i}\right)$.

Finally, it is clear that $\left(0, \ldots, 0, m-a_{n}, \ldots, m-a_{1}\right) \cap\left(K-K_{1}\right)=$ $\Phi\left(C^{\Sigma\left(m-a_{i}\right)}\right)$ and that $\Phi(0)=a$. Now we apply Mayer-Vietoris to the pairs $\{K-$ $\left.K_{1}, K-K_{2}\right\}$ and $\left\{\Phi\left(\mathrm{C}^{\Sigma\left(m-a_{i}\right)}\right), K_{3}-\Phi(0)\right\}$ :

$$
\begin{aligned}
& \left.\left.\right|_{H^{p}\left(K-K_{1} \cap K_{2}\right) \rightarrow H^{p}\left(K-K_{1}\right) \oplus H^{p}\left(K-K_{2}\right)}\right|^{p} H^{p}\left(\Phi\left(\mathbf{C}^{\Sigma\left(m-a_{i}\right)}\right)\right) \oplus H^{p}\left(K_{3}-\Phi(0)\right) \\
& \qquad H^{p}\left(K-K_{1} \cup K_{2}\right) \rightarrow H^{p+1}\left(K-K_{1} \cap K_{2}\right) \\
& \rightarrow H^{p}\left(\Phi\left(\mathbf{C}^{\Sigma\left(m-a_{i}\right)}-\{0\}\right)\right) \rightarrow H^{p+1}\left(K_{3}\right) .
\end{aligned}
$$

By (iii), (iv) and the five-lemma we get that $H^{p}\left(K-K_{1} \cup K_{2}\right) \stackrel{\Phi^{*}}{\rightarrow}$ $H^{p}\left(\mathbf{C}^{\Sigma\left(m-a_{i}\right)}-\{0\}\right)$ is an isomorphism if $p+1 \leq 2 \Sigma\left(m-a_{i}\right)$. Q.E.D.

From this point on, we assume that the integers $0 \leq a_{1} \leq \cdots \leq a_{n} \leq m$ are chosen to satisfy the additional condition that $\Sigma\left(m-a_{i}\right)=\operatorname{dim} X$. If $\bar{\delta}=\left(s_{1}\right.$, $\left.\ddot{.}, s_{a_{n}+n}\right)$ is a holomorphic section of $\bigoplus_{a_{n}+n} E$, then off the Schubert zeroes, $\mathcal{S}$ is a section of $E\left(a_{1}, \cdots, a_{n}\right)$. Let $x \in R(X)$ be an isolated Schubert zero of $\mathcal{S}$, then we define the multiplicity of the Schubert zero, denoted by zero $\left(\mathcal{S}\left(a_{1}, \cdots, a_{n}\right) ; x\right)$, as follows: let $B_{\epsilon}$ be a disc of radius $\epsilon>0$ relative to complex 
coordinates centered at $x$, let $\phi:\left.E\right|_{B \delta} \rightarrow \mathrm{C}^{m}$ be a trivialization, and let $\phi:\left.\bigoplus_{a_{n}+n} E\right|_{B \delta} \rightarrow \bigoplus_{a_{n}+n} \mathrm{C}^{m}$ be the induced trivialization of $\bigoplus_{a_{n}+n} E$. For small enough $\epsilon, \epsilon<\delta, \tilde{\phi} \circ \mathcal{S}$ maps $\partial B_{\epsilon}$ into $C^{m}\left(a_{1}, \ldots, a_{n}\right)$, and zero $\left(\mathcal{S}\left(a_{1}, \ldots, a_{n}\right) ; x\right)$ is the degree of $\tilde{\phi} \circ \mathcal{S}: \partial B_{\epsilon} \rightarrow \mathrm{C}^{m}\left(a_{1}, \ldots, a_{n}\right)$. In $\mathrm{IV}_{\mathrm{I}}$ we showed that there is a canonical choice for the generator of $H^{p}\left(\partial B_{\epsilon}\right)$ and of $H^{p}\left(\mathbf{C}^{\Sigma\left(m-a_{i}\right)}-\{0\}\right)$ for $p=2 \Sigma\left(m-a_{i}\right)-1$. Thus, via the map $\Phi: \mathbf{C}^{\Sigma\left(m-a_{i}\right)}-\{0\}$ $\rightarrow \mathrm{C}^{m}\left(a_{1}, \cdots, a_{n}\right)$ we define a canonical choice of generator for $H^{p}\left(\mathbf{C}^{m}\left(a_{1}, \cdots, a_{n}\right)\right), p=2 \Sigma\left(m-a_{i}\right)-1$, so the degree is well defined.

Let $E$ be an $m$-dimensional holomorphic hermitian vector bundle, and $0 \leq a_{1}$ $\leq \cdots \leq a_{n} \leq m$ integers. Define a $\left(\Sigma\left(m-a_{i}\right), \Sigma\left(m-a_{i}\right)\right)$ form $c\left(a_{1}, \cdots, a_{n}\right)(E)$ by $c\left(a_{1}, \ldots, a_{n}\right)(E)=\operatorname{det}\left(\left(c_{m-a_{i}-i+j}(E)\right)_{i, j=1}^{n}\right)$.

Note. The definition of $c\left(a_{1}, \ldots, a_{n}\right)(E)$ is motivated by the classical definition of the Poincaré dual to a Schubert cycle; see Hodge and Pedoe [9].

If $X$ has an exhaustion function $\tau$, and $\operatorname{dim} X=\Sigma\left(m-a_{i}\right)$, define the characteristic function $T\left(a_{1}, \ldots, a_{n}\right)(r)$ of $E$ by

$$
T\left(a_{1}, \cdots, a_{n}\right)(r)=\int_{0}^{r} \int_{X_{t}} c\left(a_{1}, \cdots, a_{n}\right)(E) d t .
$$

Let $\mathcal{S}$ be a holomorphic section of $\bigoplus_{a_{n}+n} E$, such that the Schubert zeroes of $\mathcal{S}$ of type $\left(a_{1}, \cdots, a_{n}\right)$ are isolated and contained in the regular set of $X$. Define the counting function $N\left(\mathcal{S}\left(a_{1}, \cdots, a_{n}\right) ; r\right)$ by

$$
N\left(\mathfrak{S}\left(a_{1}, \cdots, a_{n}\right) ; r\right)=\int_{0}^{r} \operatorname{zero}\left(\mathfrak{d}\left(a_{1}, \ldots, a_{n}\right) ; X_{t}\right) d t
$$

where zero $\left(\mathcal{S}\left(a_{1}, \cdots, a_{n}\right) ; X_{t}\right)=\Sigma$ zero $\left(\mathcal{S}\left(a_{1}, \cdots, a_{n}\right) ; x\right), x$ ranging over the Schubert zeroes of $\mathcal{S}$ in the interior of $X_{t}$.

Remark. If $\operatorname{dim} X=m$, and $\left(a_{1}, \cdots, a_{n}\right)=(0, m, \cdots, m)$ with $n \geq 1$, then $c\left(a_{1}, \cdots, a_{n}\right)(E)=c_{m}(E), T\left(a_{1}, \cdots, a_{n}\right)(r)^{n}=T(r)$, and $N\left(\mathcal{S}\left(a_{1}, \cdots, a_{n}\right) ; r\right)=$ $N\left(s_{1} ; r\right)$, where $T(r)$ and $N\left(s_{1} ; r\right)$ were defined in $\S \mathrm{V}$.

We would like to prove a First Main Theorem for Schubert zeroes, along the lines of Theorem 5.1, that is to find a positive form $\lambda$ of type $(p-1, p-1), p=$ $\operatorname{dim} X=\Sigma\left(m-a_{i}\right)$, such that $T\left(a_{1}, \ldots, a_{n}\right)(r)-N\left(\mathcal{S}\left(a_{1}, \cdots, a_{n}\right) ; r\right)=\int_{X_{r}} d^{c} r \wedge \lambda$ $-\int_{X_{r}} \lambda d d^{c} r$. To do this for an arbitrary bundle $E$, we would first have to prove an analogue of Theorem I, i.e. find a form $\rho$ on $E\left(a_{1}, \ldots, a_{n}\right)$ satisfying at least the following two properties:

(1) $\tilde{\pi}^{*} c\left(a_{1}, \cdots, a_{n}\right)(E)=d d^{c} \rho$ on $E\left(a_{1}, \ldots, a_{n}\right)$, where $\tilde{\pi}: \bigoplus_{a_{n}+n} E \rightarrow X$ is the projection.

(2) $\rho$ can be chosen positive on an open subbundle of $E\left(a_{1}, \cdots, a_{n}\right)$ (i.e. on an analogue of $B^{*}((E))$, if $E$ satisfies some sort of "positivity" condition.

This is a difficult problem, since even when one can find solutions to (1), as in the case $\operatorname{dim} E=n \leq 3$, there may still be no indication how to solve (2). When 
$E$ has sufficiently many sections, however, we can prove a First Main Theorem, without the analogue of Theorem I.

For the remainder of this section, we assume that $E$ has sufficiently many sections $V, \operatorname{dim} V=m+n$, and that $V$ is given a hermitian norm \|\| . The quotient bundle $Q_{n}(V)$ over $P_{n}(V)$ then has an induced norm (see proof of Lemma 4.2), and hence $E \cong e_{V}{ }^{*} Q_{n}(V)$ has an induced norm. Let $F=F\left(a_{1}, \ldots, a_{n}\right)$ be the flag manifold of all $n$-tuples $\mathcal{P}=\left(L_{a_{1}+1}, \cdots, L_{a_{n+n}}\right)$ of subspaces of $V$ such that $L_{a_{i}+1} \subset \cdots \subset L_{a_{n}+n}$ and $\operatorname{dim} L_{a_{i}+i}=a_{i}+i, 1 \leq i \leq n$. Then $F$ is a homogeneous space of $\mathrm{Gl}(V)$ and hence a complex manifold of dimension $\Sigma_{1}^{n}\left(a_{i}+1-a_{i-1}\right)$. $\left(m+n-a_{i}-i\right)$, where $a_{0}=0$. In addition $F$ is a compact symmetric homogeneous space of the isometries $I(V)$ of $V$. Let $A=\left\{(\mathcal{Q}, L) \in F \times P_{n}(V) \mid \operatorname{dim} L \cap L_{a_{i}+i}\right.$ $\geq i\}$ and consider

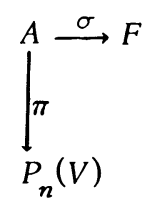

where $\sigma, \pi$ are the obvious projections.

Lemma 6.2. Let $L^{\circ}$ be a fixed n-plane in $V$, and put $\mathrm{Gl}\left(V, L^{\circ}\right)=\{g \in \mathrm{Gl}(V) \mid$ $\left.g\left(L^{\circ}\right)=L^{\circ}\right\}$. Then $(A, \pi)$ is an analytic $\mathrm{Gl}\left(V, L^{\circ}\right)$ fibre bundle over $P_{n}(V)$ with fibre $\pi^{-1}\left(L^{\circ}\right)=\left\{\mathcal{L} \in F \mid \operatorname{dim}\left(L_{a_{i}+i} \cap L^{\circ}\right) \geq i, 1 \leq i \leq n\right\}$.

Proof. Let $Y=\pi^{-1}\left(L^{\circ}\right)$, then $Y$ is a subvariety of $F$ and $\mathrm{Gl}\left(V, L^{\circ}\right)$ acts analytically on $Y$. Since $\mathrm{Gl}(V)$ is a principle analytic $\mathrm{Gl}\left(V, L^{\circ}\right)$ bundle over $P_{n}(V)$, then $(A, \pi)$ is the $Y$ associated bundle of $\mathrm{Gl}(V)$.

Lemma 6.3. Let $\bigodot^{\circ}$ be a fixed flag in $F$, and put $\mathrm{Gl}\left(\varrho^{\circ}, V\right)=\{g \in \mathrm{Gl}(V) \mid$ $\left.g\left(L_{a_{i}+i}^{\circ}\right)=L_{a_{i}+i}^{\circ}, 1 \leq i \leq n\right\}$. Then $(A, \sigma)$ is an analytic fibre bundle over $F$, with fibre $\sigma^{-1}\left(\complement^{\circ}\right)=\left(a_{1}, \cdots, a_{n}\right)\left(\varrho^{\circ}\right)$, a Scbubert cycle.

Proof. Let $Z=\left(a_{1}, \cdots, a_{n}\right)\left(\varrho^{\circ}\right)$, then $\mathrm{Gl}\left(\varrho^{\circ}, V\right)$ acts analytically on $Z$. Since $\mathrm{Gl}(V)$ is a principle analytic $\mathrm{Gl}\left(\varrho^{\circ}, V\right)$ bundle over $F$, then $(A, \sigma)$ is the $Z$ associated bundle of $\mathrm{Gl}(V)$.

Since $\operatorname{dim} A=m n+\operatorname{dim} \pi^{-1}\left(L^{\circ}\right)$ by Lemma 6.2 and $\operatorname{dim} A=\operatorname{dim} F+\sum_{i=1}^{n} a_{i}$ by Lemma 6.3 , we have

$$
\operatorname{dim} \pi^{-1}\left(L^{\circ}\right)=\operatorname{dim} F-\sum_{i=1}^{n}\left(m-a_{i}\right)=\operatorname{dim} F-\operatorname{dim} X .
$$

Hence if we define an analytic fibre bundle $\widetilde{X}$ over $X$ by 


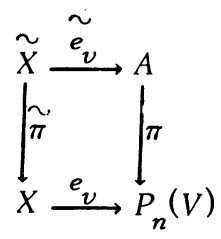

i.e. $(\tilde{X}, \tilde{\pi})=e_{V} *(A, \pi)$ then $\tilde{X}$ is an analytic space of dimension: $\operatorname{dim} X+$ $\operatorname{dim} \pi^{-1}\left(L^{\circ}\right)=\operatorname{dim} F$. Consider the obvious projection maps $\pi_{i}: F \rightarrow P_{a_{i}+i}(V)$,

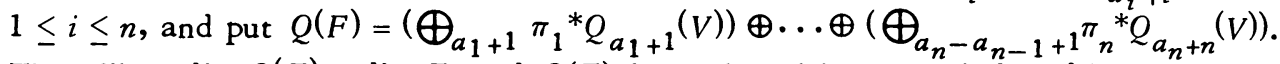
Then fibre $\operatorname{dim} Q(F)=\operatorname{dim} F$, and $Q(F)$ has a hermitian norm induced by the norms on $Q_{a_{i}+1}(V), \ldots, Q_{a_{n}+n}(V)$, and hence induced by the norm on $V$. Furthermore, $Q(F) \geq 0$, since each $Q_{a_{i}+i}(V) \geq 0$. Let $\widetilde{Q}=\widetilde{e}_{V}{ }^{*} \sigma^{*} Q(F)$, then $\widetilde{Q}$ is a positive holomorphic hermitian vector bundle over $\widetilde{X}$, and fibre $\operatorname{dim} \widetilde{Q}=\operatorname{dim} F=\operatorname{dim} \widetilde{X}$. If $s_{1}, \ldots, s_{a_{n}+n}$ are sections in $V$, then $\mathcal{S}=\left(s_{1}, \ldots, s_{a_{n}+n}\right)$ induces a holomorphic section $\widetilde{\mathcal{S}}$ of $\widetilde{Q}, \widetilde{\mathcal{S}}: \widetilde{X} \rightarrow \widetilde{Q}$, as follows: since $s_{j}$ is an element of $V$, then it induces a holomorphic section of $Q_{a_{i}+i}(V)$, for each $i$, and hence induces a holomorphic section $s_{j}^{i}$ of $e_{V}{ }^{*} \sigma^{*} \pi_{i}{ }^{*} Q_{a_{i}+i}(V)$. Then $\widetilde{\mathcal{S}}$ is the section $\left(s_{1}^{1}, \ldots, s_{a_{1+1}}^{1}\right.$, $\left.\cdots, s_{a_{n-1}+n}^{n}, \cdots, s_{a_{n+n}}^{n}\right)$ of $\widetilde{Q}^{i}$.

Lemma 6.4. Let $(x, \varrho)$ be in $\widetilde{X}$, i.e. let $e_{V}(x)$ be in $\left(a_{1}, \ldots, a_{n}\right)(£)$, then $\widetilde{S}$ bas a zero at $(x, \mathcal{P})$ if and only if $\left\langle s_{1}, \ldots, s_{a_{i}+i}\right\rangle \subseteq L_{a_{i}+i}, 1 \leq i \leq n$. Thus if the $s_{j}$ are independent in $V$, then $\mathcal{S}$ bas a zero at $(x, \mathcal{£})$ if and only if $\mathcal{S}$ bas a $S$ chubert zero at $x$ and $\mathscr{L}=\mathscr{L}(\mathcal{S})$, where $L_{a_{i}+i}(\mathcal{S})=\left\langle s_{1}, \cdots, s_{a_{i}+i}\right\rangle$.

Proof. $s_{j}^{i}(x, \mathcal{\varrho})=0 \Leftrightarrow s_{j}$ is in the $a_{i}+i$ plane $\pi_{i} \circ \sigma \circ \widetilde{e}_{V}(x, \mathcal{\varrho}) \Leftrightarrow s_{j} \in \pi_{i}$ $\circ \sigma\left(e_{V}(x), \mathfrak{Q}\right) \Leftrightarrow s_{j} \in L_{a_{i}+i}$.

Remark. This shows that if the $s_{j}$ are independent, then $\mathcal{S}$ has isolated Schubert zeroes precisely when $\widetilde{\mathcal{O}}$ has isolated zeroes.

Definition. $\mathcal{S}$ is generic if the following conditions hold:

(1) $\left\{s_{1}, \cdots, s_{a_{n}+n}\right\}$ are independent sections in $V$.

(2) The Schubert zeroes of $\mathcal{S}$ are isolated and contained in the regular set $R(X)$.

(3) If $x$ is a Schubert zero of $\mathcal{S}$, then $\operatorname{rank}\left\{s_{1}(x), \ldots, s_{a_{i}+i}(x)\right\}=a_{i}, 1 \leq i \leq n$.

Remark. We show in $\oint$ VII that almost all $\mathcal{S} \in \bigoplus_{a_{n}+n} V$ are generic.

Lemma 6.5. If $\mathcal{S}$ is generic, then the zeroes of $\widetilde{\mathcal{S}}$ are isolated and contained in $R(\widetilde{X})$, and zero $\left(\mathcal{S}\left(a_{1}, \cdots, a_{n}\right) ; x_{0}\right)=$ zero $\left(\widetilde{S} ;\left(x_{0}, \mathfrak{\varrho}(\mathscr{S})\right)\right)$, where $x_{0}$ is a Scbubert zero of $\mathcal{S}$.

Proof. Since $\mathcal{S}$ is generic, we can by renumbering assume that

$\operatorname{rank}\left\{s_{1}\left(x_{0}\right), \ldots, \widehat{s_{a_{1}+1}\left(x_{0}\right), \ldots, s_{a_{i}+i-1}\left(x_{0}\right), s_{a_{i}+i}\left(x_{0}\right)}\right\}=a_{i}, \quad 1 \leq i \leq n$, 
and that $s_{a_{i}+i}\left(x_{0}\right)=\Sigma b_{j}^{i} s_{j}\left(x_{0}\right)$, where the sum is over $1 \leq j<a_{i}+i, j \neq a_{1}+1$, $\cdots, a_{i}+i$. By standard facts about degree, using the connectivity of the general linear group, the multiplicity of the Schubert zero remains unchanged if we replace $\mathcal{S}$ by $\left(s_{1}, \ldots, s_{a_{1}}, s_{a_{1+1}}-\Sigma b_{j}^{1} s_{j} ; \cdots ; s_{a_{n-1}+n}, \cdots, s_{a_{n+n-1}}, s_{a_{n}+n}-\Sigma b_{j}^{n} s_{j}\right)$, and similarly for $\widetilde{\mathcal{S}}$. Hence we can assume that $s_{a_{i}+i}\left(x_{0}\right)=0,1 \leq i \leq n$, so that $e_{V}\left(x_{0}\right)=\left\langle s_{a_{1+1}}, \cdots, s_{a_{n+n}}\right\rangle$. Choose $s_{a_{n+n+1}}, \cdots, s_{m+n}$ in $V$ such that $\left\{s_{1}, \cdots\right.$, $\left.s_{m+n}\right\}$ is a basis for $V$. Let $z^{i}=\left(z_{1}^{i}, \ldots, \widehat{z_{a_{1+1}}^{i}}, \ldots, z_{a_{n+n-1}}^{a^{2}}, \widehat{z_{a_{n}+n}}, z_{a_{n+n+1}}^{i}\right.$, $\left.\cdots, z_{m+n}^{i}\right) \in \mathbf{C}^{m}$, and put $z=\left(z^{1}, \ldots, z^{n}\right) \in \mathbf{C}^{m n}$, then the map of $\mathbf{C}^{m n}$ into $P_{n}(V)$ by $z \rightarrow L(z)=\left\langle s_{a_{1+1}}+\Sigma z_{j}^{1} s_{j}, \ldots, s_{a_{n+n}}+\Sigma z_{j}^{n} s_{j}\right\rangle$ gives holomorphic coordinates centered at $e_{V}\left(x_{0}\right)$. Put $u_{j}(z)=s_{j}$ if $j \neq a_{1}+1, \cdots, a_{n}+n$ and $u_{a_{i}+i}(z)=$ $s_{a_{i}+i}+\Sigma z_{j}^{i} s_{j}, 1 \leq i \leq n$, then for each $z,\left\{u_{1}(z), \ldots, u_{m+n}(z)\right\}$ is a basis for $V$. Let $w_{j}=\left(w_{a_{i+i+1}}^{j}, \cdots, w_{m+n}^{j}\right) \in \mathrm{C}^{m+n-a_{i}-i}$, when $a_{i-1}+i \leq j \leq a_{i}+i$, and put $w=\left(w^{1}, \ldots, w^{a_{n}+n}\right) \in \mathbf{C}^{\operatorname{dim} F}$. Then the map from $\mathbf{C}^{\operatorname{dim} F} \rightarrow F$, given by $w \rightarrow$ $\mathcal{L}(z, w)$, for fixed $z \in \mathbf{C}^{m n}$, gives holomorphic coordinates in $F$, where $\mathcal{L}(z, w)=$ $\left(L_{a_{1+1}}(z, w), \ldots, L_{a_{n+n}}(z, w)\right) \in F, L_{a_{i+i}}(z, w)=\left\langle L_{a_{i-1+i-1}}(z, w), u_{a_{i-1+i}}(z)+\right.$ $\left.\sum w_{j}^{a_{i-1+i}} u_{j}(z), \cdots, u_{a_{i}+i}(z)+\Sigma w_{j}^{a_{i+i}} u_{j}(z)\right\rangle$. The map $(z, w) \rightarrow(L(z), \mathcal{L}(z, w))$ gives holomorphic coordinates in $P_{n}(v) \times F$ centered at $\left(e_{V}\left(x_{0}\right), \mathfrak{L}(\mathcal{S})\right)$ since the Jacobian of this map is easily seen to be never zero.

Note. $L(z)=\left\langle u_{a_{1+1}}(z), \cdots, u_{a_{n}+n}(z)\right\rangle$ hence $(L(z), \mathcal{L}(z, w)) \in A$ if and only if $w_{j}^{a_{i+i}}=0$ for $j \neq a_{i}+i, \cdots, a_{n}+n$, and $a_{i}+i<j \leq m+n$. If we put $\widetilde{w}=w$, subject to the condition that $w_{j}^{a_{i}+i}=0$ for $a_{i}+i<j \leq m+n, j \neq a_{i}+i, \cdots, a_{n}+n$, then $(z, \widetilde{w}) \rightarrow(L(z), \mathcal{L}(z, \widetilde{w}))$ gives holomorphic coordinates in $A$ centered at $\left(e_{V}\left(x_{0}\right), \mathcal{L}(\mathcal{S})\right)$. Since $s_{a_{1+1}}\left(x_{0}\right)=\cdots=s_{a_{n}+n}\left(x_{0}\right)=0$ the $s_{j}$, with $j \neq a_{i}+i$, form a frame for $E$ in a neighborhood $U$ of $x_{0}$, and we can find holomorphic functions $f_{j}^{i}$ on $U$, such that $s_{a_{i+i}}=-\Sigma f_{j}^{i} s_{j}$, the sum over $j \neq a_{1}+1, \cdots, a_{n}+n$. If in addition, $U$ is a coordinate neighborhood, then $(x, \widetilde{w}) \rightarrow(x, \mathcal{Q}(f(x), \widetilde{w}))$ gives holomorphic coordinates in $\widetilde{X}$ centered at $\left(x_{0}, \mathcal{\complement}(\mathcal{S})\right)$. We can further assume that $x_{0}$ is the only Schubert zero of $\mathcal{S}$ in $U$. If we trace through all the identifications and apply Proposition 6.1, then it is clear that zero $\left(S\left(a_{1}, \ldots, a_{n}\right) ; x_{0}\right)=$ $\operatorname{deg}\left(f_{a_{1+2}}^{1}, \cdots, f_{m+n}^{1} ; \cdots ; f_{a_{n}+n+1}^{n}, \cdots, f_{m+n}^{n}\right)$ at $x_{0}$, where $f_{j}^{i}$ is only defined for $j \neq a_{1}+1, \cdots, a_{n}+n$, so there are $\Sigma\left(m+n-a_{i}-i\right)-\Sigma(n-i)=\Sigma\left(m-a_{i}\right)$ of the $f_{j}^{i}$ s here.

Consider the section $u_{j}^{i}$ of $e_{V}{ }^{*} \sigma{ }^{*} \pi_{i}{ }^{*} Q_{a_{i+i}}(V)$ defined by $u_{j}^{i}(x, \widetilde{w})=$ $u_{j}^{i}(f(x)) / L_{a_{i+i}}(f(x), \widetilde{w})$. Then $\left\{u_{a_{i+i+1}}, \cdots, u_{m+n}^{i}\right\}$ is a frame of $e_{V}{ }^{*} \sigma \pi_{i} Q_{a_{i}+i}(V)$. Now $s_{j}=u_{j}$ if $j \neq a_{i}+i, 1 \leq i \leq n$, hence $s_{j}^{i}=u_{j}^{i}$. But if $a_{i-1}+i \leq j \leq a_{i}+i-1$, then $s_{j}^{i}(x, \widetilde{w})=-\Sigma w_{k}^{j} u_{k}^{i}(x, \widetilde{w})$, since $u_{j}(z)+\sum w_{k}^{j} u_{k}(z) \in L_{a_{i}+i}(z, w)$. Denote by $\Sigma_{j=p}^{\prime}$ the sum over $p \leq j \leq q, j \neq a_{1}+1, \ldots, a_{n}+n$. Since $u_{a_{i+i}}(f(x))=s_{a_{i+i}}+$ $\Sigma^{\prime} f_{j}^{i}(x) s_{j}$, then 


$$
\begin{aligned}
s_{a_{i}+i}^{i}(x, \widetilde{w})= & u_{a_{i}+i}^{i}(x, \widetilde{w})-\sum_{j=1}^{m+n} f_{j}^{i}(x) s_{j}^{i}(x, \widetilde{w}) \\
= & -\sum w_{k}^{a_{i}^{+i}} u_{k}^{i}(x, \widetilde{w})-\sum_{j=1}^{a_{i}^{+i-1}} f_{j}^{i}(x) s_{j}^{i}(x, w)-\sum_{j=a_{i}+i+1}^{m+n} f_{j}^{i}(x) u_{j}^{i}(x, \tilde{w}) \\
= & -\sum_{k=i+1}^{n} w_{a_{k}+k}^{a_{i}+i} u_{a_{k}+k}^{i}(x, \tilde{w})-\sum_{j=1}^{a_{i}+i-1} f_{j}^{i}(x) s_{j}^{i}(x, \widetilde{w}) \\
& -\sum_{j=a_{i}+i+1}^{m+n} f_{j}^{i}(x) u_{j}^{i}(x, \tilde{w})
\end{aligned}
$$

note $w_{k}^{a_{i+i}}=0$ if $k \neq a_{i+1}+i+1, \ldots, a_{n}+n$. We define a homotopy $\widetilde{\mathcal{S}}(x, \widetilde{w}, t)$ into $\widetilde{Q}$ by $s_{j}^{i}(x, \widetilde{w}, t)=s_{j}^{i}(x, \widetilde{w})$ if $a_{i-1}+i \leq j \leq a_{i}+i-1$, and

$$
s_{a_{i}+i}^{i}(x, \tilde{w}, t)=u_{a_{i}+i}^{i}(x, \widetilde{w})-t \sum_{j=1}^{a_{i}+i-1} f_{j}^{i}(x) s_{j}^{i}(x, \widetilde{w})-\sum_{j=a_{i}+i+1}^{m+n} f_{j}^{i}(x) u_{j}^{i}(x, \widetilde{w}) .
$$

If $\widetilde{\mathcal{S}}(x, \widetilde{w}, t)=0$, then $s_{j}^{i}(x, \widetilde{w})=0$ if $a_{i-1}+i \leq \jmath \leq a_{i}+i-1$, which implies that $s_{j}^{k}(x, \widetilde{w})=0$ if $1 \leq j<a_{k}+k, j \neq a_{1}, \ldots, a_{n}+n$, so that $s_{a_{i}+i}^{i}(x, \widetilde{w}, t)=$ $s_{a_{i}+i}^{s_{i}}(x, \widetilde{w}, 1)=s_{a_{i+i}}^{i}(x, \widetilde{w})$. But then $\widetilde{\mathcal{S}}(x, \widetilde{w})=0$, which happens only at $\left(x_{0}, 0\right)$, hence $\widetilde{\mathcal{S}}(x, \widetilde{w}, t)$ is zero only for $(x, \widetilde{w})=\left(x_{0}, 0\right)$. For purposes of computing the order of the zero we can replace $\widetilde{S}(x, \widetilde{w})$ by

$$
\begin{aligned}
& \widetilde{S}(x, \tilde{w}, 0)=\left(-\sum w_{k}^{1} u_{k}^{1}(x, \tilde{w}), \cdots,-\sum w_{k}^{a} u_{k}^{1}(x, \tilde{w}),-\sum_{k=2}^{n} w_{a_{k}+k}^{a_{1}+1} u_{a_{k}+k}^{1}(x, \widetilde{w})\right. \\
& -\sum_{j=a_{1}+2}^{m+n} f_{j}^{1}(x) u_{j}^{1}(x, \widetilde{w}) ; \cdots ;-\sum w_{k}^{a n-1+n} u_{k}^{n}(x, \widetilde{w}), \cdots, \\
& \left.-\sum w_{k}^{a_{n}+n-1} u_{k}^{n}(x, \tilde{w}),-\sum_{j=a_{n}+n-1}^{m+n} f_{j}^{n}(x) u_{j}^{n}(x, \tilde{w})\right) .
\end{aligned}
$$

Finally, using the frames $\left\{u_{a_{i+i}+1}^{i}, \cdots, u_{m+n}^{i}\right\}$ of $e_{V}{ }^{*} \sigma{ }^{*} \pi_{i}{ }^{*} Q_{a_{i}+i}(V)$ to give the obvious frame for $\widetilde{Q}$, and by then permuting the frame, we see that zero $\left(\mathcal{S},\left(x_{0}, \varrho(\mathcal{S})\right)\right)$ is the degree of the map $(x, \widetilde{w}) \rightarrow\left(\left(f_{a_{1+2}}^{1}, \cdots, f_{m+n}^{1} ; \cdots ; f_{a_{n}+n+1}^{n}\right.\right.$, $\left.\left.\cdots, f_{m+n}^{n}\right), \widetilde{w}\right)$ which is the same as the degree of the map $x \rightarrow\left(f_{a_{1}+2}^{1}, \cdots, f_{m+n}^{1}\right.$; $\left.\cdots ; f_{a_{n}+n+1}^{n+n}, \cdots, f_{m+n}^{n}\right)$. Hence zero $\left(\mathcal{S} ;\left(x_{0}, \mathcal{S}(\mathcal{S})\right)\right)=\operatorname{zero}\left(\mathcal{S}\left(a_{1}, \cdots, a_{\widetilde{C}^{n}}\right) ; x_{0}\right)$. Q.E.D.

In order to investigate the relationship between $c_{p}(\widetilde{Q}), p=\operatorname{dim} \widetilde{Q}$, and $c\left(a_{1}, \cdots, a_{n}\right)(E)$, we must first discuss the fibre integral. Let $\pi: Y^{m} \rightarrow Z^{n}$ be an analytic fibre bundle with compact fibre $W^{k}, k=m-n$, where $Y, Z, W$ are all 
analytic spaces. The fibre integral $\pi_{*}: A^{p, q}(Y) \rightarrow A^{p-k, q-k}(Z)$ is the adjoint of $\pi^{*}$, that is $\int_{Y} \psi \wedge \pi^{*} \phi=\int_{Z} \pi_{*} \psi \wedge \phi$ where $\psi \in A^{p, q}(Y), \phi \in A^{m-p, m-q}(Z)$ and $\phi$ has compact support. The fibre integral is defined as follows: by a partition of unity argument it suffices to consider the case $Y=Z \times W$, where $Z$ is a subvariety of a domain $U_{1}$ in $\mathbf{C}^{r}, W$ is a subvariety of a domain $U_{2}$ in $\mathbf{C}^{s}$, and $\psi \in A^{p, q}(Y)$ has compact support. Let $z_{1}, \cdots, z_{n}$ be coordinates in $\mathrm{C}^{n} ; w_{1}, \cdots, w_{s}$ coordinates in $\mathrm{C}^{s}$, then $\psi$ has an extension $\tilde{\psi} \in A^{p, q}\left(U_{1} \times U_{2}\right), \psi=$ $\sum \psi_{I J K L}(z, w) d w^{K} d \bar{w}^{L} d z^{I} d \bar{z}^{J}$ where the $\psi_{I J K L}$ are smooth. Then $\pi_{*} \psi$ is the restriction to $Z$ of

$$
\sum_{\substack{|I|=p-k,|J|=q-k \\|K|=k,|L|=k}}\left(\int_{W} \psi_{I J K L}(z, w) d w^{K} d \bar{w}^{L}\right) d z^{I} d \bar{z}^{J},
$$

which is a smooth form. The adjoint property is then clear. If $f: Z^{\prime} \rightarrow Z$ is holomorphic, and we consider the diagram

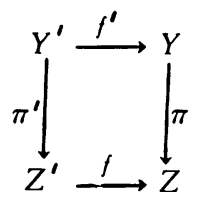

where $Y^{\prime}=f^{*} Y$, then it is also clear that $\pi_{*}^{\prime} \circ\left(f^{\prime}\right)^{*}=f^{*} \circ \pi_{*}$. Note. The fibre integral exists under very general conditions, see [4], [10], [12].

Lemma 6.6. Let $p=\operatorname{dim} \widetilde{X}=\operatorname{dim} \widetilde{Q}$, then $c\left(a_{1}, \cdots, a_{n}\right)(E)=\widetilde{\pi}_{*} c_{p}(\widetilde{Q})$.

Proof. Consider the diagram

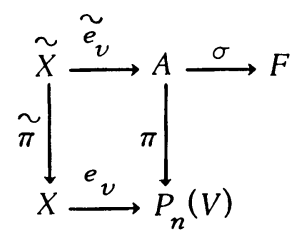

then $c\left(a_{1}, \cdots, a_{n}\right)(E)=e_{V}{ }^{*} c\left(a_{1}, \cdots, a_{n}\right)\left(Q_{n}(V)\right)$, and $c_{p}(\widetilde{Q})=e_{V}{ }^{*} \sigma{ }^{*} c_{p}(Q(F))$, so we need only show that $c\left(a_{1}, \cdots, a_{n}\right)\left(Q_{n}(V)\right)=\pi_{*} \sigma^{*} c_{p}(Q(F))$. Let $g$ be an element of $I(V)$, the isometries of $V$, then $g$ acts on $P_{n}(V)$, on $F$, and on $A$, and the action of $g$ commutes with $\pi$ and $\sigma$, so $g{ }^{*} \pi_{*} \sigma{ }^{*} c_{p}(Q(F))=\pi_{*} g{ }^{*} \sigma{ }^{*} c_{p}(Q(F))=$ $\pi_{*} \sigma^{*} c_{p}\left(g^{*} Q(F)\right)$. But $g^{*} Q(F) \cong Q(F)$ as hermitian bundles, so $c_{p}\left(g^{*} Q(F)\right)=$ $c_{p}(Q(F))$ and $\pi_{*} \sigma^{*} c_{p}(Q(F))$ is invariant under $I(V)$. Similarly, $g^{*} c\left(a_{1}, \cdots, a_{n}\right)\left(Q_{n}(V)\right)$ $=c\left(a_{1}, \ldots, a_{n}\right)\left(g^{*} Q_{n}(V)\right)=c\left(a_{1}, \ldots, a_{n}\right)\left(Q_{n}(V)\right)$ so $c\left(a_{1}, \cdots, a_{n}\right)\left(Q_{n}(V)\right)$ is invariant under $I(V)$. Since $P_{n}(V)$ is a symmetric homogeneous space of $I(V)$, then it suffices to show $c\left(a_{1}, \cdots, a_{n}\right)\left(Q_{n}(V)\right)=\pi_{*} \sigma^{*} c_{p}(Q(F))$ as cohomology classes. Let $\mathcal{L}^{\circ} \in F$, with $L_{a_{i}+i}^{\circ}=\left\langle v_{1}, \cdots, v_{a_{i}+i}\right\rangle$ and put $s_{j}=s_{v_{j}}$, the section of 
$Q_{n}(V)$ induced by $v_{j}$. Then $\mathcal{S}$ induces a section $\widetilde{\mathcal{S}}=\left(s_{1}^{1}, \ldots, s_{a_{1}+1}^{1} ; \cdots\right.$; $\left.s_{a_{n-1}^{n}+n}^{n}, \cdots, s_{a_{n+n}}^{n}\right)$ of $Q(F)$, and $\widetilde{\mathcal{S}}$ has a zero only at $\mathcal{L}^{\circ}$. By taking local coordinates in $F$, i.e. $w \rightarrow \mathcal{\complement}(0, w)$ in the notation of Lemma 6.5 , it is easy to check that zero $\left(\tilde{\mathcal{S}}, \mathfrak{L}^{\circ}\right)=1$, and by applying the Generalized Gauss-Bonnet Theorem 4.1, we see that $\int_{F} c_{p}(Q(F))=1$. We show that $\pi_{*} \sigma{ }^{*} c_{p}(Q)$ is Poincaré dual to $\left(a_{1}, \cdots, a_{n}\right)\left(\complement^{\circ}\right) \subset P_{n}(V)$, i.e. if $\phi$ is a closed $(q, q)$ form on $P_{n}(V), q=a_{1}+\cdots+a_{n}$, we must show that

$$
\int\left(a_{1}, \cdots, a_{n}\right)(£ \circ)=\int_{P_{n}(V)} \pi_{*} \sigma^{*} c_{p}(Q(F)) \wedge \phi .
$$

By fibre integrating,

$$
\int_{P_{n}(V)} \pi_{*} \sigma^{*} c_{p}(Q(F)) \wedge \phi=\int_{A} \sigma^{*} c_{p}(Q(F)) \wedge \pi^{*} \phi=\int_{F} c_{p}(Q(F)) \sigma_{*} \pi^{*} \phi .
$$

But $\phi$ is closed, and from the adjoint property it is clear that fibre integration commutes with exterior derivative so $d \sigma_{*} \pi^{*} \phi=\sigma_{*} \pi^{*} d \phi=0$, i.e. $\sigma_{*} \pi^{*} \phi$ is a constant function on $F$. Since $\sigma_{*} \pi^{*} \phi \equiv\left(\sigma_{*} \pi^{*} \phi\right)\left(\bigodot^{\circ}\right)$, and $\int_{F} c_{p}(Q(F))=1$, then

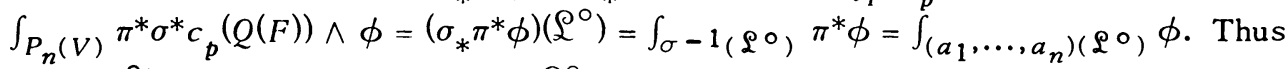
$\pi_{*} \sigma^{*} c_{p}(\widetilde{Q})$ is dual to $\left(a_{1}, \cdots, a_{n}\right)\left(\bigodot^{\circ}\right)$. But from algebraic geometry, we know the dual of $\left(a_{1}, \ldots, a_{n}\right)\left(\mathcal{Q}^{\circ}\right)$ is $c\left(a_{1}, \ldots, a_{n}\right)\left(Q_{n}(V)\right)$, see Hodge and Pedoe [9].

Remark. The fibre integral of a positive form is clearly positive, so we have shown that $Q\left(a_{1}, \cdots, a_{n}\right)(E) \geq 0$, since $\widetilde{Q}$ is a positive bundle.

Let $X$ have an exhaustion $r$, then by fibre integrating the First Main Theorem on $\tilde{X}$, we get the desired result on $X$.

Proposition 6.7 (First Main Theorem for Schubert Zeroes). Let $\mathcal{S}=\left(s_{1}, \cdots, s_{a_{n}+n}\right)$ be generic, with $\left\|s_{j}\right\| \leq 1 / a_{n}+n$ as elements in $V, 1 \leq j \leq a_{n}+n$. Then

$$
T\left(a_{1}, \cdots, a_{n}\right)(r)-N\left(\mathcal{S}\left(a_{1}, \cdots, a_{n}\right) ; r\right)=\frac{1}{4 \pi} \int_{\partial X_{r}} d^{c} \tau \wedge \lambda-\frac{1}{4 \pi} \int_{X} \lambda d d^{c} \tau,
$$

where $\lambda=\widetilde{\pi}_{*} \widetilde{\mathcal{S}} *_{\rho}(\widetilde{Q})$, for almost all $r$. If $\tau$ is pseudo-concave, then $N\left(\mathscr{S}\left(a_{1}, \cdots, a_{n}\right) ; r\right)$ $\leq T\left(a_{1}, \cdots, a_{n}\right)(r)+C$, and if $c\left(a_{1}, \ldots, a_{n}\right)(E) \not \equiv$ then $0 \leq \delta\left(\mathcal{S}\left(a_{1}, \cdots, a_{n}\right)\right) \leq 1$, where

$$
\delta\left(\mathcal{S}\left(a_{1}, \ldots, a_{n}\right)\right)=1-\overline{\lim }\left\{N\left(\mathcal{S}\left(a_{1}, \ldots, a_{n}\right) ; r\right) / T\left(a_{1}, \ldots, a_{n}\right)(r)\right\} .
$$

Proof. Let $\tilde{\tau}=\tau \circ \tilde{\pi}$, then $\tilde{\tau}$ is an exhaustion, and $\tilde{\tau}$ is pseudo-concave if $\tau$ is pseudo-concave. The norm $N(\widetilde{\delta}) \leq \Sigma N\left(s_{j}^{i}\right) \leq \Sigma_{k=1}^{a_{n}+n}\left\|s_{k}\right\| \leq 1$, and the zeroes of $\widetilde{\mathcal{J}}$ are isolated and contained in the regular set of $\overline{\widetilde{X}}$.

We apply Theorem 5.1 to $\widetilde{S}$ and get that, for almost all $r$,

$$
T(r)-N(r, \widetilde{\mathcal{S}})=\frac{1}{4 \pi} \int_{\partial \widetilde{X}_{r}} d^{c} \tilde{\tau} \wedge \lambda-\frac{1}{4 \pi} \int_{\tilde{X}_{r}} \tilde{\lambda}_{d d^{c}} \tilde{\tau}
$$




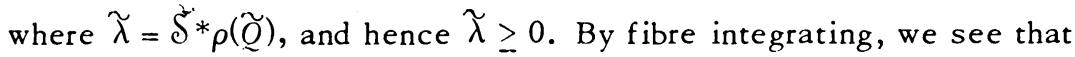

$$
\begin{aligned}
T(r) & =\int_{0}^{r} \int_{\tilde{X}_{t}} c_{p}(Q) d t=\int_{0}^{r} \int_{X} \tilde{\pi}_{t} c_{p}(\tilde{Q}) d t \\
& =\int_{0}^{r} \int_{X_{t}} c\left(a_{1}, \cdots, a_{n}\right)(E) d t=T\left(a_{1}, \ldots, a_{n}\right)(r) ;
\end{aligned}
$$

and

$$
N(\widetilde{\mathcal{S}}, r)=\int_{0}^{r} \text { zero }\left(\widetilde{\mathcal{S}} ; \widetilde{X}_{t}\right) d t=\int_{0}^{r} \text { zero }\left(\mathfrak{S}\left(a_{1}, \cdots, a_{n}\right) ; X_{t}\right)=N\left(\mathcal{S}\left(a_{1}, \cdots, a_{n}\right) ; r\right)
$$

by Lemma 6.5. Furthermore,

$$
\int_{\partial \tilde{X}_{r}} d^{c} \tilde{\tau} \wedge \tilde{\lambda}=\int_{\partial \tilde{X}_{r}} \tilde{\pi}^{*} d^{c} \tau \wedge \tilde{\lambda}=\int_{X_{r}} d^{c} \tau \wedge \tilde{\pi}_{*} \tilde{\lambda}
$$

and

$$
\int_{\tilde{X}_{r}} \tilde{\lambda} d d^{c} \tau=\int_{\tilde{X}_{r}} \tilde{\lambda} \wedge \tilde{\pi}^{*} d d^{c} \tau=\int_{X_{r}} \tilde{\pi}_{*} \tilde{\lambda} \wedge d d^{c} \tau .
$$

If $\tilde{\tau}$ is pseudo-concave, then $N(r, \widetilde{\mathcal{S}}) \leq T(r)+c$ by Corollary 5.5, so $N\left(\mathcal{S}\left(a_{1}, \cdots, a_{n}\right) ; r\right) \leq T\left(a_{1}, \cdots, a_{n}\right)(r)+c$, and the deficiency satisfies $0 \leq$ $\delta\left(\mathcal{S}\left(a_{1}, \ldots, a_{n}\right)\right) \leq 1$.

VII. Value distribution theory of Schubert zeroes. Thus far we have considered the distribution of Schubert zeroes for only one set of sections $\mathcal{S}$ at a time. In this section we show that if $X$ is pseudo-concave then almost all $\mathcal{S}$ have the same number of Schubert zeroes.

Theorem 7.1. Let $X$ be an irreducible complex space with pseudo-concave exbaustion $\tau$. Let $E$ be a bolomorphic vector bundle of fibre dimension $m$ over $X$, and $V$ a vector space of holomorphic sections of $E, \operatorname{dim} V=m+n$, such that $e_{x}: V \rightarrow E_{x}$ is onto at each point $x \in X$. Let $\mathcal{S}=\left(s_{1}, \ldots, s_{a_{n}+n}\right)$ be an element of $\bigoplus_{a_{n}+n} V$, where $s_{1}, \ldots, s_{a_{n+n}}$ are bolomorpbic sections, independent in $V$, and $\left(a_{1}, \cdots, a_{n}\right)$ is a fixed set of integers, $0 \leq a_{1} \leq \cdots \leq a_{n} \leq m$, such that $\Sigma\left(m-a_{i}\right)=$ $\operatorname{dim} X$. If $c\left(a_{1}, \ldots, a_{n}\right)(E) \equiv 0$, then almost all $\mathcal{S}$ bave the same number of $S c h u-$ bert zeroes of type $\left(a_{1}, \ldots, a_{n}\right)$, i.e. $\delta\left(\mathcal{S}\left(a_{1}, \cdots, a_{n}\right)\right)=0$ for almost all $\mathfrak{S} \in \bigoplus_{a_{n+n}} V$.

Remark. Almost all $\mathcal{S}$ consist of independent sections in $V$, so we can restrict our attention to such $\mathcal{S}$. If $\mathcal{S}, \mathcal{S}^{\prime}$ consist of independent sections, then we say $\mathcal{S}$ is equivalent to $\mathcal{S}^{\prime}$ if $\mathcal{L}(\mathcal{S})=\mathfrak{L}\left(\mathcal{S}^{\prime}\right)$. We have already seen in $\S$ VI that the set of equivalence classes is in $1-1$ correspondence with $F$, and if $\mathcal{S}, \mathcal{S}^{\prime}$ are equivalent then $N\left(\mathcal{S}\left(a_{1}, \cdots, a_{n}\right) ; r\right)=N\left(\mathcal{S}^{\prime}\left(a_{1}, \ldots, a_{n}\right) ; r\right)$ so we define $N\left(\left(a_{1}, \ldots, a_{n}\right)(\mathcal{S}) ; r\right)=$

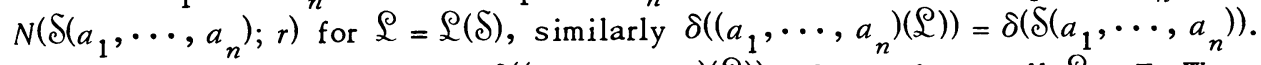
Hence it suffices to show that $\delta\left(\left(a_{1}, \ldots, a_{n}\right)(\mathcal{Q})\right)=0$ for almost all $\mathcal{Q} \in F$. We define $\mathfrak{L}$ generic if $\mathcal{L}=\mathfrak{L}(\mathfrak{S})$ for $\mathfrak{S}$ generic; this is well defined since $\mathfrak{S}$ equivalent 
to $\mathcal{S}^{\prime}$, and $\mathcal{S}$ generic, implies $\mathcal{S}^{\prime}$ is generic. Then Theorem 7.1 follows from the following three propositions.

Proposition 7.2. $\mathcal{S}$ is generic for almost all $\mathfrak{S} \in \bigoplus_{a_{n}+n} V$, or equivalently, $\mathfrak{Q}$ is generic for almost all $\mathcal{Q} \in F$. In fact $\mathcal{S}$ bas only $S$ chubert zeroes of multiplicity 1 for almost all $\mathcal{S}$, i.e. $e_{V}$ bits $\left(a_{1}, \cdots, a_{n}\right)(£)$ transversely for almost all $£ \in F$.

Proposition 7.3. If $\omega$ denotes the invariant normalized volume form on $F$, then $\int_{F} N\left(\left(a_{1}, \cdots, a_{n}\right)(£) ; r\right) \omega(£)=T\left(a_{1}, \ldots, a_{n}\right)(r)$.

Proposition 7.4. For each $\&$ generic in $F$, there is a constant $c_{\mathfrak{Q}} \geq 0$ such

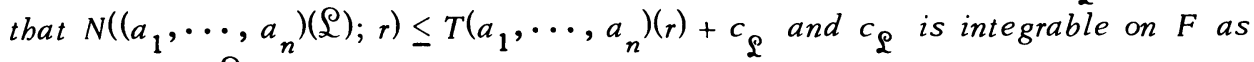
a function of $\mathcal{Q}$.

Proof of Theorem 7.1. We denote $\delta\left(\left(a_{1}, \cdots, a_{n}\right)(£)\right), N\left(\left(a_{1}, \cdots, a_{n}\right)(£) ; r\right)$ and $T\left(a_{1}, \cdots, a_{n}\right)(r)$ by $\delta(£), N(£)$ and $T$ respectively. By Proposition 7.4, we have $0 \leq \delta(£) \leq 1$ for almost all $\mathcal{\complement}$. But by Fatou's Lemma,

$$
\begin{aligned}
& \int_{F} \delta(£) \omega(£) \leq \int_{F} \lim \left(1-N(£) / T+c_{\S} / T\right) \omega(£)
\end{aligned}
$$

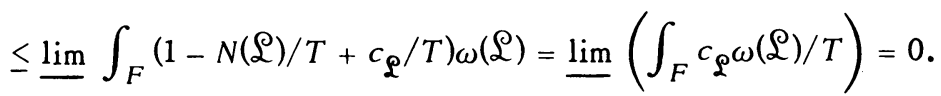

Therefore $\int_{F} \delta(£) \omega(£) \leq 0$ which implies $\delta(£)=0$ for almost all $\mathcal{~}$.

Proof of Proposition 7.2. Let $Y_{1} \subset \tilde{X}$ be the set $\{(x, \varrho) \in \widetilde{X} \mid$ $\operatorname{dim}\left(e_{V}(x) \cap L_{a_{i}+i}\right)<i$ for some $\left.i, 1 \leq i \leq n\right\}$. Then $Y_{1}$ is a subvariety of $\tilde{X}$ of codimension $\geq 1$. Let $Y_{2} \subset R(\tilde{X})-Y_{1}$ be the set $\left\{(x, \mathcal{I}) \in R(\tilde{X})-Y_{1} \mid \mathcal{I}=\mathscr{I}(\mathcal{S})\right.$, where $\widetilde{\mathcal{S}}$ is not transversal to the zero section of $\widetilde{Q}$. Then by Lemma 6.4 ,

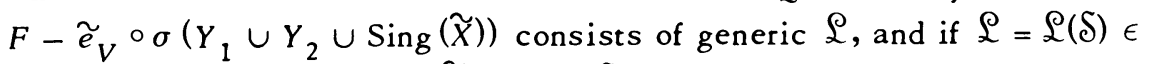
$F-\widetilde{e}_{V} \circ \sigma\left(Y_{1} \cup Y_{2} \cup \operatorname{Sing}(\tilde{X})\right)$, then $\mathcal{S}$ has nondegenerate Schubert zeroes and $e_{V}$ is transversal to $\left(a_{1}, \cdots, a_{n}\right)\left(()\right.$. If $Y_{2}$ is a subvariety of $R(\tilde{X})-Y_{1}$ of codim $Y_{2}=1$, then $Y_{1} \cup Y_{2} \cup \operatorname{Sing}(\tilde{X})$ is a countable union of submanifolds each of dimension less than $\operatorname{dim} \widetilde{X}=\operatorname{dim} F$, and hence $\tilde{e}_{V} \circ \sigma\left(Y_{1} \cup Y_{2} \cup \operatorname{Sing}(\widetilde{X})\right)$ has measure zero in $F$.

Using the notation of the proof of Lemma 6.5 , we consider the map $(x, \widetilde{w}) \rightarrow$ $(x, \mathfrak{L}(f(x), \widetilde{w}))$. We take the coordinates $w \rightarrow \mathfrak{Q}(0, w)$ in $F$ and compute the Jacobian of $\widetilde{e}_{V} \circ \sigma:(x, \widetilde{w}) \rightarrow \mathscr{\complement}(f(x), \widetilde{w})$ relative to the coordinates $(x, \widetilde{w})$ in $R(\tilde{X})-Y_{1}$, and it is clear from the proof of Lemma 6.5 that the Jacobian is nonsingular at $\left(x_{0}, 0\right)$ if and only if the map $x \rightarrow\left(f_{a_{1+2}}^{1}(x), \ldots, f_{m+n}^{1}(x) ; \cdots\right.$; $\left.f_{a_{n}+n+1}^{n}(x), \cdots, f_{m+n}^{n}(x)\right)$ is nonsingular at $x_{0}$, i.e. if and only if $\widetilde{\mathcal{S}}$ is transversal to the zero section of $\widetilde{Q}$ at $\left(x_{0}, 0\right)$. Thus $Y_{2}$ is the zero set of the determinant of $\left.d\left(\widetilde{e}_{V} \circ \sigma\right)\right|_{R(\tilde{X})-Y_{1}}$. If $R(\tilde{X})-Y_{1}$ is connected, then either $Y_{2}=R(\tilde{X})-Y_{1}$ or $Y_{2}$ has codimension 1. But if $Y_{2}=R(\tilde{X})-Y_{1}$, then for $p=\operatorname{dim} F, c_{p}(\widetilde{Q})=$ $\widetilde{e}_{V}{ }^{*} \sigma^{*} c_{p}(Q(F))=0$ on $R(\widetilde{X})-Y_{1}$ and hence on $\tilde{X}$, implying that $c\left(a_{1}, \cdots, a_{n}\right)(E)$ 
$=\tilde{\pi}_{*} c_{p}(\widetilde{Q})=0$ on $X$ contrary to assumption. Hence $Y_{2}$ has codimension 1 if $R(\widetilde{X})-Y_{1}$ is connected, thus we need only show $\widetilde{X}$ is irreducible. Since the fibres of $(A, \sigma)$ are Schubert cycles and are irreducible, then $A$ is irreducible. But this implies that the fibres of $(A, \pi)$ are irreducible, hence $\widetilde{X}$ is irreducible. Thus $\tilde{e}_{V} \circ \sigma\left(Y_{1} \cup Y_{2} \cup \operatorname{Sing}(\widetilde{X})\right)$ has measure zero and almost all $\mathcal{L}$ are generic.

Proof of Proposition 7.3. It clearly suffices to show that

$$
\int_{F} \operatorname{zero}\left(\left(a_{1}, \cdots, a_{n}\right)(£) ; X_{r}\right) \omega(£)=\int_{X_{r}} c\left(a_{1}, \cdots, a_{n}\right)(E),
$$

where

$$
\operatorname{zero}\left(\left(a_{1}, \cdots, a_{n}\right)(£) ; X_{r}\right) \equiv \operatorname{zero}\left(\mathcal{S}\left(a_{1}, \cdots, a_{n}\right) ; X_{r}\right)
$$

for any $\mathcal{S}$ such that $\mathcal{L}=\mathscr{L}(\mathcal{S})$. By Proposition 7.2 , for almost all $\mathcal{L}$. zero $\left(\left(a_{1}, \ldots, a_{n}\right)(\mathscr{Q}) ; X_{r}\right)$ is the number of times a neighborhood of $\mathscr{L}$ is covered by $\sigma \circ \widetilde{e}_{V} \mid \tilde{X}_{r}$. Thus $\int_{F}$ zero $\left(\left(a_{1}, \ldots, a_{n}\right)(£) ; X_{r}\right) \omega(£)=\int_{X_{r}} \widetilde{e}_{V}{ }^{*} \sigma^{*} \omega$. Now $\omega=$ $c_{p}(Q(F))$ was shown in the proof of Lemma 6.6, thus $\int_{X_{r}} c\left(a_{1}, \ldots, a_{n}\right)(E)=$ $\int \tilde{X}_{r} c_{p}(\widetilde{Q})=\int \tilde{X}_{r} \tilde{e}_{V}{ }^{*} \sigma^{*} c_{p}(Q)$.

Proof of Proposition 7.4. Given $\mathfrak{L}^{\circ} \in F$, assume we can find a neighborhood $W$ of $\mathfrak{L}^{\circ}$ in $F$ such that to each $\mathscr{\varrho} \in W$ there corresponds $\mathcal{S}(\mathcal{L}) \in \bigoplus_{a_{n}+n} V$ with the following properties:

(i) $\mathfrak{L}(\mathcal{S}(\mathfrak{Q}))=\mathfrak{Q}$.

(ii) If $\mathcal{S}(£)=\left(s_{1}(£), \cdots, s_{a_{n}+n}(£)\right)$ then $\left\|s_{j}(£)\right\| \leq 1 / a_{n}+n$ as elements in $V, 1 \leq j \leq a_{n}+n$.

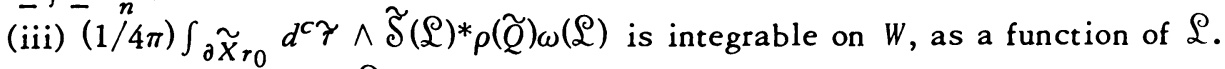
By Proposition 6.7, if $\mathcal{Q} \in W$ is generic, then

$$
\begin{aligned}
& N\left(\left(a_{1}, \cdots, a_{n}\right)(£) ; r\right) \leq T\left(a_{1}, \ldots, a_{n}\right)(r)+\frac{1}{4 \pi} \int_{\tilde{x}_{r_{0}}} \widetilde{\mathcal{S}}(\varrho) * \rho(\tilde{Q}) d d^{c} \tilde{\tau} \\
& =T\left(a_{1}, \cdots, a_{n}\right)(r)+\frac{1}{4 \pi} \int_{\tilde{X}_{r_{0}}} d^{c} \tilde{\tau} \wedge \tilde{\mathcal{S}}(\mathcal{Q}) * \rho(\tilde{Q}) \\
& +N\left(\left(a_{1}, \ldots, a_{n}\right)(£) ; r_{0}\right)-T\left(a_{1}, \ldots, a_{n}\right)\left(r_{0}\right) \text {. }
\end{aligned}
$$

Therefore

$$
\begin{aligned}
N\left(\left(a_{1}, \ldots, a_{n}\right)(£) ; r\right) \leq & T\left(a_{1}, \ldots, a_{n}\right)(r)+\frac{1}{4 \pi} \int_{\partial \tilde{X}_{r_{0}}} d^{c} \tilde{\tau} \wedge \widetilde{S}(£) * \rho(\tilde{Q}) \\
& +N\left(\left(a_{1}, \cdots, a_{n}\right)(£) ; r_{0}\right) .
\end{aligned}
$$

But $N\left(\left(a_{1}, \cdots, a_{n}\right)(£) ; r_{0}\right)$ is integrable on $W$ by Proposition 7.3 , and therefore putting 


$$
c_{\mathfrak{Q}}=\frac{1}{4 \pi} \int_{\partial \tilde{X}_{r_{0}}} d^{c} \tilde{\tau} \wedge \widetilde{\mathcal{S}}(\varrho) * \rho(\tilde{Q})+N\left(\left(a_{1}, \ldots, a_{n}\right)(£) ; r_{0}\right)
$$

we have $N\left(\left(a_{1}, \ldots, a_{n}\right)(£) ; r\right) \leq T\left(a_{1}, \ldots, a_{n}\right)(r)+c_{\S}$ where $c_{£} \omega(£)$ is integrable on $W$. The proposition then clearly follows from a partition of unity argument. Note that $d^{c} \widetilde{\tau} \wedge \widetilde{\mathcal{S}}(\mathscr{Q}) * \rho(\widetilde{Q})$ is positive on $\partial \widetilde{X}_{r_{0}}$.

To construct $\mathcal{S}(£)$, let $v_{1}, \cdots, v_{m+n}$ be an orthonormal basis of $V$ such that $L_{a_{i}+i}^{\circ}=\left\langle v_{1}, \cdots, v_{a_{i+i}}\right\rangle, 1 \leq i \leq n$. Coordinates in a neighborhood of $\mathcal{Q}^{\circ}$ in $F$ are given by $z \rightarrow \mathfrak{Q}(z)$, where $z=\left(z^{1}, \cdots, z^{a_{n}+n}\right), z^{j}=\left(z_{a_{i+i+1}^{j}}^{j}, \cdots, z_{m+n}^{j}\right)$ for $a_{i-1}+i \leq j \leq a_{i}+i$, and

$$
L_{a_{i}+i}(z)=\left\langle L_{a_{i-1}+i-1}(z), v_{a_{i-1}+i}+\sum z_{j}^{a_{i-1}+i} v_{j}, \cdots, v_{a_{i}+i}+\sum z_{j}^{a_{i}^{+i}} v_{j}\right\rangle .
$$

Put $\mathcal{S}(z)=1 / p\left(s_{1}(z), \cdots, s_{a_{n}+n}(z)\right)$ where $s_{j}(z)=v_{j}+\sum z_{k}^{j} v_{k}$ and $p$ is a large constant. Then we can find neighborhoods $W, U$ of $\mathcal{L}^{\circ}$, with $\bar{U}$ contained in the coordinate patch such that $\bar{W} \subset U$ and $\delta(z)$ satisfies (ii) on $U$. Now we can also consider $\widetilde{\mathcal{S}}(z)$ as a section of $Q(F)$, so that $\widetilde{\mathcal{S}}(\mathcal{Q}) * \rho(\widetilde{Q})=\widetilde{e}_{V}{ }^{*} \sigma * \widetilde{\mathcal{S}}(z) * \rho(Q(F))$.

We wish to show $\int_{W}\left(\int_{\partial} \widetilde{X}_{r_{0}} d^{c} \widetilde{\tau} \wedge \widetilde{e}_{V} * \sigma * \widetilde{S}(z)^{*} \rho(Q(F))\right)_{\omega}(z)$ is finite. Let $H_{1}=\left(\widetilde{e}_{V} \circ \sigma\right)^{-1}(U) \cap \partial \widetilde{X}_{r_{0}}$ and $H_{2}=\left(\widetilde{e}_{V} \circ \sigma\right)^{-1}(F-U) \cap \partial \widetilde{X}_{r_{0}}$. Since $\tilde{e}_{V} *{ }^{*} \widetilde{\mathcal{S}}(z) * \rho(Q(F)) \wedge \omega(z)$ is a $C^{\infty}$ form on the compact set $\bar{W} \times H_{2}$, it suffices to show that $\int_{W}\left(\int_{H_{1}} d^{c} \widetilde{\tau} \wedge \widetilde{e}_{V}{ }^{*} \sigma * \widetilde{\mathcal{S}}(z) * \rho(Q(F))\right)_{\omega}(z)$ is finite.

Consider the section $s_{k}^{j}$ of $\pi_{j}^{*} Q_{a_{i+i}}(V)$ on $U$, by $s_{k}^{j}=v_{k} / L_{a_{j+j}}(z)$ for $k=a_{j}+j+1, \cdots, m+n$. Then the $s_{k}^{j}$ form a frame of $\pi_{j}{ }^{*} Q_{a_{j}+j}(V)$ on $U$, and hence we get the obvious frame for $Q(F)=\left(\bigoplus_{a_{1+1}} \pi_{1}{ }^{*} Q_{a_{1+1}}(V)\right) \oplus \cdots \oplus$

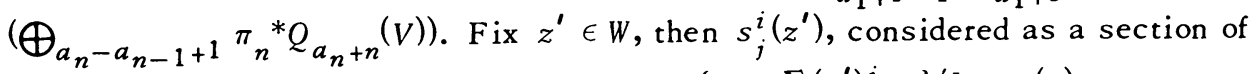
$\pi_{i}{ }^{*} Q_{a_{i}+i}(V)$, evaluated at the point $z \in U$ is $\left\{v_{j}+\Sigma\left(z^{\prime}\right)_{k}^{j} v_{k}\right\} / L_{a_{i}+i}(z)=$ $\left(\Sigma\left(z^{\prime}-z\right)_{k}^{j} v_{k}\right) / L_{a_{i}+i}(z)=\Sigma\left(z^{\prime}-z\right)_{k}^{j} s_{k}^{i}$, when $a_{i-1}+i \leq j \leq a_{i}+i$. Thus from the proof of Lemma 5.3, we have

$$
\begin{aligned}
& \widetilde{\widetilde{S}}\left(z^{\prime}\right) * \rho(Q(F)) \\
& \quad=\sum\left(\log \left\|z^{\prime}-z\right\|^{2} \phi_{I J K L}+\psi_{I J K L}\right)(-1)^{|K|+|L|}\left\|z^{\prime}-z\right\|^{-|K|-|L|_{d z^{I}} d z^{J} d z^{K} d z^{L},}
\end{aligned}
$$

where $\phi_{I J K L}, \Psi_{I J K L}$ are bounded functions of $z \in \bar{U}, z^{\prime} \in W$.

We can apply Fubini's Theorem since $d^{c} \widetilde{\tau} \wedge{\widetilde{e}}_{V}{ }^{*} \sigma^{*} \widetilde{\mathcal{S}}\left(z^{\prime}\right) * \rho(Q(F)) \omega(z)$ is positive on $H_{1} \times W$, and we have

$$
\begin{aligned}
\int_{W}\left(\int_{H_{1}} d^{c} \tilde{\tau} \wedge \tilde{e}_{V}^{*} \sigma^{*} \widetilde{\mathcal{E}}\left(z^{\prime}\right) * \rho(Q(F))\right) \omega\left(z^{\prime}\right) \\
=\int_{H_{1}} d^{c} \tilde{\tau} \tilde{e}_{V}^{*} \sigma^{*}\left(\int_{z^{\prime} \in W} \widetilde{\mathcal{S}}\left(z^{\prime}\right) \rho(Q(F)) \omega\left(z^{\prime}\right)\right) .
\end{aligned}
$$


Since the $\phi_{I J K L}, \Psi_{I J K L}$ are bounded functions, we will be done if $\int_{W}\left(\log \left\|z^{\prime}-z\right\|^{2}\right)\left\|z^{\prime}-z\right\|^{-|K|-|L|} \omega\left(z^{\prime}\right)$ and $\int_{W}\left\|z^{\prime}-z\right\|^{-|K|-|L|} \omega\left(z^{\prime}\right)$ are bounded functions of $z \in U$. But $\omega(z)=\alpha(z) d z d \bar{z}$ where $\alpha$ is a bounded function of $z \in \bar{U}$ and $d z=d z_{1} \cdots d z_{q}, q=\operatorname{dim} F$. Now

$$
\begin{aligned}
& \int_{W}\left(\log \left\|z^{\prime}-z\right\|^{2}\right)\left\|z^{\prime}-z\right\|^{-|K|-|L|} d z^{\prime} d \bar{z}^{\prime} \\
& =\int_{z^{\prime}+z \in W}\left(\log \left\|z^{\prime}\right\|^{2}\right)\left\|z^{\prime}\right\|^{-|K|-|L|_{d z^{\prime} d \bar{z}^{\prime}}}
\end{aligned}
$$

which is bounded since $d z^{\prime} d \bar{z}^{\prime}$ is of the order of $\left\|z^{\prime}\right\|^{2 q-1}$, and $|K|+|L| \leq 2 q-2, q=\operatorname{dim} F$. Q.E.D.

Using the equivalence between Schubert zeroes and Schubert cycles we can rephrase Theorem 7.1 as follows:

Theorem 7.5. Let $X$ be an irreducible complex space with pseudo-concave exbaustion $\tau$, and let $f: X \rightarrow P_{n}(V)$ be a holomorphic map, where $V$ is an $m+n$ dimensional complex vector space; $P_{n}(V)$ is the Grassmann manifold of $n$-planes in $V$. Fix integers $0 \leq a_{1} \leq \cdots \leq a_{n} \leq m$ such that $\operatorname{dim} \widetilde{X}=\Sigma\left(m-a_{i}\right)$. Then $f$ bits almost all Schubert cycles of type $\left(a_{1}, \cdots, a_{n}\right)$ the same number of times,

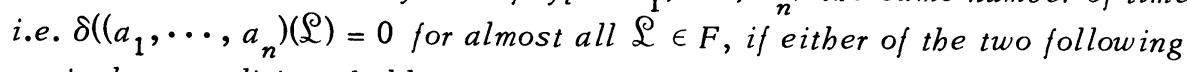
equivalent conditions bold:

(1) $f^{*} c\left(a_{1}, \cdots, a_{n}\right)\left(Q_{n}(V)\right)$ is not identically zero on $X$.

(2) $f$ is transversal to $\left(a_{1}, \cdots, a_{n}\right)(£)$ at a point $x_{0} \in X$, for some $£$ in $F$.

\section{BIBLIOGRAPHY}

1. T. Bloom and M. Herrera, De Rham cohomology of an analytic space, Invent. Math. 7 (1969), 275-296. MR $40 \# 1601$.

2. R. Bott and S. S. Chern, Hermitian vector bundles and the equidistribution of the zeroes of their holomorphic sections, Acta Math. 114 (1965), 71-112. MR 32 \#3070.

3. S. S. Chern, Complex manifolds without potential theory, Van Nostrand Math. Studies, no. 15, Van Nostrand, Princeton, N. J., 1967. MR 37 \#940.

4. H. Federer, Geometric measure theory, Die Grundlehren der math. Wissenschaften, Band 153, Springer-Verlag, New York, 1969。 MR 41 \#1976。

5. H. Flanders, Differential forms with applications to the physical sciences, Academic Press, New York, 1963. MR 28 \#5397.

6. P. A. Griffiths, Hermitian differential geometry, Chern classes, and positive vector bundles, Global Analysis (Papers in Honor of K. Kodaira), Univ。 Tokyo Press, Tokyo, 1969, pp. 185-251. MR $41 \# 2717$.

7. H. Hironaka, Resolution of singularities of an algebraic variety over a field of characteristic zero. I, II, Ann。 of Math. (2) 79 (1964), 109-203. MR 33 \#7333.

8. J. Hirschfelder, The first main theorem of value distribution in several variables, Invent Math. 8 (1969), 1-33. MR 39 \#7146. 
9. W. V. D. Hodge and D. Pedoe, Methods of algebraic geometry. Vol. II. Book III: General the ory of algebraic varieties in projective space. Book IV: Quadrics and Grassmann varieties, Cambridge Univ. Press, Cambridge, 1952. MR 13, 972.

10. J. King, The currents defined by analytic varieties, Acta Math. 127 (1971), 185-220.

11. P. Lelong, Intégration sur un ensemble analytique complexe, Bull. Soc. Math. France 85 (1957), 239-262. MR 20 \#2465.

12. W. Stoll, Value distribution of holomorphic maps into compact complex manifolds, Lecture Notes in Math., vol. 135, Springer-Verlag, Berlin and New York, 1970. MR 42 \#2040.

13. G. Stolzenberg, Volumes, limits, and extensions of analytic varieties, Lecture Notes in Math., no. 19, Springer-Verlag, Berlin and New York, 1966. MR 34 \#6156.

14. H. Wu, Remarks on the first main theorem in equidistribution theory. I-IV, J. Differential Geometry 2 (1968), 197-202, 369-384; ibid. 3 (1969), 83-94, 433-466. MR $43 \# 2247$ a, b, c, d.

DEPARTMENT OF MATHEMATICS, PRINCETON UNIVERSITY, PRINCETON, NEW JERSEY 08540 